\title{
Machine learning in medicine
}

Citation for published version (APA):

Bennis, F. C. (2020). Machine learning in medicine: big pictures require small, but crucial strokes.

[Doctoral Thesis, Maastricht University]. Maastricht University. https://doi.org/10.26481/dis.20201113fb

Document status and date:

Published: 01/01/2020

DOI:

10.26481/dis.20201113fb

Document Version:

Publisher's PDF, also known as Version of record

\section{Please check the document version of this publication:}

- A submitted manuscript is the version of the article upon submission and before peer-review. There can be important differences between the submitted version and the official published version of record.

People interested in the research are advised to contact the author for the final version of the publication, or visit the DOI to the publisher's website.

- The final author version and the galley proof are versions of the publication after peer review.

- The final published version features the final layout of the paper including the volume, issue and page numbers.

Link to publication

\footnotetext{
General rights rights.

- You may freely distribute the URL identifying the publication in the public portal. please follow below link for the End User Agreement:

www.umlib.nl/taverne-license

Take down policy

If you believe that this document breaches copyright please contact us at:

repository@maastrichtuniversity.nl

providing details and we will investigate your claim.
}

Copyright and moral rights for the publications made accessible in the public portal are retained by the authors and/or other copyright owners and it is a condition of accessing publications that users recognise and abide by the legal requirements associated with these

- Users may download and print one copy of any publication from the public portal for the purpose of private study or research.

- You may not further distribute the material or use it for any profit-making activity or commercial gain

If the publication is distributed under the terms of Article $25 \mathrm{fa}$ of the Dutch Copyright Act, indicated by the "Taverne" license above, 


\section{Machine learning in medicine}

Big pictures require small, but crucial strokes

Frank Bennis 
Lay-out: $\quad$ Frank Bennis

Cover design: $\quad$ Ben Hermans

Printed by: Gildeprint

ISBN: $\quad$ 978-94-6419-023-6

Copyright (C) 2020 by Frank Bennis

All rights reserved. No part of this thesis may be reproduced, stored or transmitted in any form or by any means without prior permission of the author.

Cover figure is downloaded from freepik.com and adjusted by Ben Hermans

Financial support for the publication of this thesis as provided by Maastricht University is gratefully acknowledged. 


\title{
Machine learning in medicine
}

\author{
Big pictures require small, but crucial strokes
}

\section{Proefschrift}

ter verkrijging van de graad van doctor aan de Universiteit Maastricht, op gezag van de Rector Magnificus, prof. dr. Rianne M. Letschert, volgens het besluit van het College van Decanen, in het openbaar te verdedigen

op vrijdag 13 november 2020 om 14:00 uur.

door

Franciscus Cornelis Bennis

geboren op 23 juli 1991

te Zwaag, Nederland 


\section{Promotores}

Prof. dr. T. Delhaas

Prof. dr. B.W. Kramer

\section{Copromotor}

Dr. P. Andriessen (Máxima Medisch Centrum Veldhoven)

\section{Beoordelingscommissie}

Prof. dr. ir. R.L.M. Peeters (voorzitter)

Prof. dr. J.C.C. van der Horst

Prof. dr. W.H. Mess

Prof. dr. T.W. Orlikowsky (Uniklinik RWTH Aachen)

Prof. dr. ir. P.H. Veltink (Universiteit Twente) 


\section{Table of Contents}

$\begin{array}{lll}\text { Chapter } 1 \quad \text { General Introduction } & 6\end{array}$

$\begin{array}{lll}\text { Chapter } 2 & \text { Artifacts in pulse transit time measurements using standard } & 14\end{array}$ patient monitoring equipment

Chapter 3 Ratio of arterial blood pressures at borders of window 26 surrounding systolic peak indicates patent ductus arteriosus in preterm infants

Chapter 4 Improving prediction of favourable outcome after six months in 42 patients with severe traumatic brain injury using physiological cerebral parameters in a multivariable logistic regression model

Chapter 5 A machine-learning based analysis for the recognition of 60 progressive central hypovolemia

Chapter 6 Support vector machine based monitoring of 74 cardio-cerebrovascular reserve during simulated hemorrhage

Appendices

$\begin{array}{lll}\text { A. } & \text { Summary } & 114 \\ \text { B. } & \text { Valorisation } & 120 \\ \text { C. } & \text { Dankwoord / Acknowledgements } & 126 \\ \text { D. } & \text { Curriculum Vitae } & 132\end{array}$ 



\section{Chapter 1}

General Introduction 
8 | Chapter 1 


\subsection{Background}

\subsubsection{Machine learning models in medicine}

For diagnostic, interventional or predictive purposes, medical doctors constantly try to identify patterns in patients' vital signs. However, a clinician can only interpret a limited number of parameters at the same time. Furthermore, it is difficult for a clinician to assess trends in data. An example is found during surgical procedures, where the anesthesiologist aims to keep blood volume constant using blood pressure and heart rate as parameters $[1,2]$. Although absolute blood pressure and heart rate values may lie in a physiological range, the combination of a larger number of parameters may indicate progression towards hypovolemia [3]. Machine learning models can assist the clinician by performing bed-side diagnostics or outcome prediction based on the combination of large amounts of current and trend data. Since machine learning models may outperform clinicians and do not suffer from stress, disease, fatigue or emotion-based decision making, they are developed more and more for clinical purposes [4-7]. However, application of machine learning models lags behind, with only a handful of models being actually implemented [8]. This may partly be due a lack of trust by the clinician in the accuracy of such potential non-transparent models [9-11]. Since the prediction of a model may define whether treatment or even life support is given or withdrawn, a wrong prediction may have tremendous consequences. In this thesis, we address three (of the many more) crucial steps in the development and assessment of machine learning in medicine: raw data assessment, parameter extraction and model creation.

\subsubsection{Data quality}

"On two occasions I have been asked, "Pray, Mr. Babbage, if you put into the machine wrong figures, will the right answers come out?” ... I am not able rightly to apprehend the kind of confusion of ideas that could provoke such a question." [12]

It is tempting to think of a machine learning model as a magical machine. Charles Babbage (mathematician and inventor of the difference engine, precursor of electronic computers) already recognized in the 19th century that the performance of a model greatly depends on data quality [12]. This kind of model behavior is captured by the well-known phrase "garbage in, garbage out”.

Clinical data contains noise, artifacts and missing data since data collection is usually a byproduct of clinical care [13-15]. An example of the noisiness of clinical data is found in the measurement of invasive arterial blood pressure. During data collection, patients are nursed and treated and undergo scans and physical examinations that, in addition to measurement artifacts from the sensor itself, affect data quality [16]. As follows from the phrase "garbage in, garbage out", noise in data collection leads to noise in estimated parameters, which results in decreased model performance [17-19]. Therefore, assessment of data quality is fundamental in the development of clinical models. 


\subsubsection{Parameter extraction}

Raw clinical data have to be converted to meaningful parameters before they can be used in common models. Although it is possible to feed raw data into complex models, such an approach is not transparent and underlying biological and physiological mechanisms are hard to interpret $[9,20]$. The development of such parameters is often based on domain knowledge. Parameters are extracted by the researcher based on proven or suspected relationships between data and outcome, reflecting biology-based medicine [21,22]. In the example of the invasive blood pressure measurement, the raw data is the continuous blood pressure waveform. If the outcome to predict is whether a neonatal patient is or will become septic or not, domain knowledge dictates that mean blood pressure over a period of time would be a meaningful parameter, as well as heart rate characteristics, entropy and many more [2325]. Such parameters can be extracted using common analysis software. Good parameters carry the information needed for the model to generate accurate predictions. Without good parameters, a model has no information to base its prediction on, resulting in inaccurate predictions $[26,27]$. The construction of high-quality parameters is therefore a fundamental step in model creation. In addition, by defining parameters based on physiology, it is possible to understand which mechanisms in the human body relate to the outcome [11], since the model in which the parameter is inserted may give feedback on the relative importance of the parameters. Therefore, identification of interpretable parameters predictive of the outcome is important in the development of clinical models.

\subsubsection{Model creation}

Parameters are used to train machine learning models and subsequently predict outcomes in new patients. Machine learning models are diverse in complexity, ranging from logistic regression to deep neural networks [28,29]. A simple model such as logistic regression gives in numerous instances good performance with a high transparency enabling physiological interpretation of the parameters [30]. If the parameter is also indicative of the underlying physiology, a link can be made between physiology and prediction. Furthermore, acceptability of these linear models in a clinical situation is high due to their high transparency $[8,30]$. However, the relationship between input and output that they can model is simple. If the underlying physiological relationship is more complex, the model may not be able to fit accordingly, leading to a lower performance. More complex models such as neural networks can fit complex relationships [31,32]. Therefore, these models may result in a better performance in case of more complex underlying physiology. However, with increasing complexity comes decreasing transparency, leading to a model in which it is unknown what happens internally (black-box model), resulting in decreased acceptability in clinical situations $[8,32,33]$. Although models such as neural networks can model more complex relationships, they do not always outperform simple models such as logistic regression [3436]. 


\subsection{Thesis outline}

This thesis aims to analyze the importance of raw data assessment, parameter extraction and model creation in the context of currently unresolved clinical problems.

The pulse transit time is measured as the time difference between the R peak in the ECG and the upslope of the photoplethysmogram and may be used as a parameter in clinical models [37]. As two different devices measure the ECG and the photoplethysmogram, synchronization may have an effect on the calculation of the pulse transit time. Because correct parameters are crucial for model creation, we performed a raw data assessment study in Chapter 2, where we investigated the effect of synchronization errors on the calculation of the pulse transit time.

The ductus arteriosus is an artery between the aorta and the pulmonary artery that is open during fetal life, but should close after birth. In preterm infants, the ductus arteriosus may remain open and can have a hemodynamically significant impact [38]. Though an echocardiogram can detect the presence of a patent ductus arteriosus, it is a discontinuous measurement, while the ductus arteriosus may open, close and reopen over time. Therefore, in Chapter 3 we extracted a physiology-based parameter that can discriminate patients with from patients without a patent ductus arteriosus.

The outcome in patients with traumatic brain injury admitted to the intensive care unit ranges from death to full recovery [39]. It is helpful for clinicians and family to have an indication of a patient's outcome as early as possible. To this end, we created in Chapter 4 a logistic regression model to predict outcome after six months in traumatic brain injury patients admitted to the intensive care unit using clinical data at admittance and of the first 24 hour thereafter.

Patients may lose a lot of blood during surgery. If uncompensated for, this may eventually lead to syncope (if the patient is conscious), cerebral hypoxia and/or ischemia [40]. Blood pressure declines after severe blood loss, but it is known that blood pressure may remain stable during initial blood loss. Therefore, in Chapter 5 and 6, we train machine-learning models to detect pending syncope in central hypovolemia-induced subjects using a support vector machine model and a neural network model.

In the final chapter of this thesis, Chapter 7 , the findings of the various chapters are put into broader perspective in a general discussion. 


\subsection{References}

1. Orlinsky M, Shoemaker W, Reis ED, Kerstein MD. Current controversies in shock and resuscitation. Surg Clin North Am. 2001;81(6):1217-62.

2. Hamilton-Davies C, Mythen MG, Salmon JB, Jacobson D, Shukla A, Webb AR. Comparison of commonly used clinical indicators of hypovolaemia with gastrointestinal tonometry. Intensive Care Med. 1997;23(3):276-81.

3. Moulton SL, Mulligan J, Grudic GZ, Convertino VA. Running on empty? The compensatory reserve index. J Trauma Acute Care Surg. 2013;75(6):1053-9.

4. Obermeyer Z, Emanuel EJ. Predicting the Future - Big Data, Machine Learning, and Clinical Medicine. N Engl J Med. 2016 ;375(13):1216-9.

5. Yu K-H, Zhang C, Berry GJ, Altman RB, Ré C, Rubin DL, et al. Predicting non-small cell lung cancer prognosis by fully automated microscopic pathology image features. Nat Commun. 2016;7(1):12474.

6. Ehteshami Bejnordi B, Veta M, Johannes van Diest P, van Ginneken B, Karssemeijer N, Litjens G, et al. Diagnostic Assessment of Deep Learning Algorithms for Detection of Lymph Node Metastases in Women With Breast Cancer. JAMA. 2017;318(22):2199210.

7. Gulshan V, Peng L, Coram M, Stumpe MC, Wu D, Narayanaswamy A, et al. Development and Validation of a Deep Learning Algorithm for Detection of Diabetic Retinopathy in Retinal Fundus Photographs. JAMA. 2016;316(22):2402-10.

8. He J, Baxter SL, Xu J, Xu J, Zhou X, Zhang K. The practical implementation of artificial intelligence technologies in medicine. Nat Med. 2019;25(1):30-6.

9. Wang F, Casalino LP, Khullar D. Deep Learning in Medicine-Promise, Progress, and Challenges. JAMA Intern Med. 2019;179(3):293-4.

10. ElNaqaI, RuanD, ValdesG,DekkerA,McNuttT,GeY, etal.Machinelearning and modeling: Data, validation, communication challenges. Med Phys. 2018;45(10):e834-e840.

11. Vellido A. The importance of interpretability and visualization in machine learning for applications in medicine and health care. Neural Comput Appl. 2019;1-15.

12. Babbage C. Difference Engine No.1. In: Passages from the Life of a Philosopher. Cambridge University Press; 1864. p. 41-67.

13. Cios KJ, William Moore G. Uniqueness of medical data mining. Artif Intell Med. 2002;26(1-2):1-24.

14. Hersh WR, Weiner MG, Embi PJ, Logan JR, Payne PRO, Bernstam EV., et al. Caveats for the Use of Operational Electronic Health Record Data in Comparative Effectiveness Research. Med Care. 2013;51(2):S30-7.

15. Kim H-S, Kim D-J, Yoon K-H. Medical Big Data Is Not Yet Available: Why We Need Realism Rather than Exaggeration. Endocrinol Metab. 2019;34(4):349-54.

16. McGhee BH, Bridges EJ. Monitoring arterial blood pressure: what you may not know. Crit Care Nurse. 2002;22(2):60-79.

17. Nettleton DF, Orriols-Puig A, Fornells A. A study of the effect of different types of noise on the precision of supervised learning techniques. Artif Intell Rev. 2010;33(4):275306.

18. Atla A, Tada R, Sheng V, Singireddy N. Sensitivity of different machine learning algorithms to noise. J Comput Sci Coll. 2011;26(5):96-103. 
19. Kalapanidas E, Avouris N, Craciun M, Neagu D. Machine Learning Algorithms: A study on noise sensitivity. Proc 1st Balcan Conf Informatics. 2003:356-65.

20. Hinton G. Deep Learning-A Technology With the Potential to Transform Health Care. JAMA. 2018;320(11):1101-2.

21. Foster KR, Koprowski R, Skufca JD. Machine learning, medical diagnosis, and biomedical engineering research - commentary. Biomed Eng Online. 2014;13(94):1-9.

22. Guyon I, Elisseeff A. An Introduction to Variable and Feature Selection. J Mach Learn Res. 2003:1157-82.

23. Lake DE, Richman JS, Griffin MP, Moorman JR. Sample entropy analysis of neonatal heart rate variability. Am J Physiol Integr Comp Physiol. 2002;283(3):R789-97.

24. Moorman JR, Lake DE, Griffin MP. Heart Rate Characteristics Monitoring for Neonatal Sepsis. IEEE Trans Biomed Eng. 2006;53(1):126-32.

25. Wynn JL, Wong HR. Pathophysiology and Treatment of Septic Shock in Neonates. Clin Perinatol. 2010;37(2):439-79.

26. Domingos P. A few useful things to know about machine learning. Commun ACM. 2012;55(10):78-87.

27. Deo RC. Machine Learning in Medicine. Circulation. 2015;132(20):1920-30.

28. Hastie T, Tibshirani R, Friedman J. The Elements of Statistical Learning: Data Mining, Inference, and Prediction. Second. New York: Springer-Verlag; 2009.

29. Beam AL, Kohane IS. Big Data and Machine Learning in Health Care. JAMA. 2018;319(13):1317-8.

30. Shipe ME, Deppen SA, Farjah F, Grogan EL. Developing prediction models for clinical use using logistic regression: an overview. J Thorac Dis. 2019;11(S4):S574-84.

31. Bishop CM. Neural networks for pattern recognition. Oxford: Clarendon Press; 1995.

32. Tu J V. Advantages and disadvantages of using artificial neural networks versus logistic regression for predicting medical outcomes. J Clin Epidemiol. 1996;49(11):1225-31.

33. Topol EJ. High-performance medicine: the convergence of human and artificial intelligence. Nat Med. 2019;25(1):44-56.

34. Christodoulou E, Ma J, Collins GS, Steyerberg EW, Verbakel JY, Van Calster B. A systematic review shows no performance benefit of machine learning over logistic regression for clinical prediction models. J Clin Epidemiol. 2019;110:12-22.

35. Kurt I, Ture M, Kurum AT. Comparing performances of logistic regression, classification and regression tree, and neural networks for predicting coronary artery disease. Expert Syst Appl. 2008;34(1):366-74.

36. Song JH, Venkatesh SS, Conant EA, Arger PH, Sehgal CM. Comparative analysis of logistic regression and artificial neural network for computer-aided diagnosis of breast masses. Acad Radiol. 2005;12(4):487-95.

37. Foo JYA, Wilson SJ. Clinical applications of pulse transit time in paediatric critical care. J Med Eng Technol. 2009;33(1):79-86.

38. Dice JE, Bhatia J. Patent ductus arteriosus: an overview. J Pediatr Pharmacol Ther. 2007;12(3):138-46.

39. Maas AI, Stocchetti N, Bullock R. Moderate and severe traumatic brain injury in adults. Lancet Neurol. 2008;7(8):728-41.

40. Secher NH, Van Lieshout JJ. Normovolaemia defined by central blood volume and venous oxygen saturation. Clin Exp Pharmacol Physiol. 2005;32(11):901-10. 



\section{Chapter 2}

\section{Artifacts in pulse transit time measurements using standard patient monitoring equipment}

Frank C. Bennis, Carola van Pul, Jarno J.L. van den Bogaart, Peter Andriessen, Boris W. Kramer, Tammo Delhaas

PLoS One. 2019;14(6):eo218784 


\begin{abstract}

\section{Background}

Pulse transit time (PTT) refers to the time it takes a pulse wave to travel between two arterial sites. PTT can be estimated, amongst others, using the electrocardiogram (ECG) and photoplethysmogram (PPG). Because we observed a sawtooth artifact in the PTT while using standard patient monitoring equipment for ECG and PPG, we explored the reasons for this artifact.
\end{abstract}

\title{
Methods
}

PPG and ECG were simulated at a heartrate of both 100 and 160 beats per minute while using a Masimo PPG post-processing module and a Philips patient monitor setup at the neonatal intensive care unit. Two different post-processing modules were used. PTT was defined as the difference between the R-peak in the ECG and the point of 50\% increase in the PPG.

\section{Results}

A sawtooth artifact was seen in all simulations. Both length (59.2 to $72.4 \mathrm{~s}$ ) and amplitude (30.8 to $36.0 \mathrm{~ms}$ ) of the sawtooth were dependent on the post-processing module used. Furthermore, the absolute PTT value differed up to $250 \mathrm{~ms}$ depending on post-processing module and heart rate. The sawtooth occurred because the PPG wave continuously showed a minimal prolongation during the length of the sawtooth, followed by a sudden shortening. Both artifacts were generated in the post-processing module containing Masimo algorithms.

\section{Conclusion}

Post-processing of the PPG signal in the Masimo module of the Philips patient monitor introduces a sawtooth in PPG and derived PTT. This sawtooth, together with a large moduledependent absolute difference in PTT, renders the thus-derived PTT insufficient for clinical purposes. 


\subsection{Introduction}

Pulse transit time (PTT) is commonly determined as the time difference between onset of cardiac ejection (approximated by the R peak in the electrocardiogram (ECG)) and the arrival of the pulse in a finger as determined from the photoplethysmogram (PPG) [1-4]. Increases in PTT are related to changes in the cardiovascular system such as lower systolic blood pressure, lower arterial stiffness or an increased pathway length [5-8]. PTT might be used, amongst others, to detect sleep disordered breathing [9] or as a surrogate measure of blood pressure due to the correlation of PTT with blood pressure [10]. Another promising application of PTT is monitoring of closure of the ductus arteriosus in a neonatal setting $[11,12]$. However, when we started our study on the applicability of PTT to detect the status of the ductus arteriosus, the estimated PTT showed a remarkable and relatively large sawtoothlike artifact (Figure 2.1). This artifact might not only impede clinical usefulness of PTT in the detection of a patent ductus arteriosus, but also the use of PTT as a surrogate measure of blood pressure because it reflects non-existing sudden changes in blood pressure. In the present study we explored why this sawtooth artifact occurred in PTTs obtained with standard patient monitoring equipment for ECG and PPG.

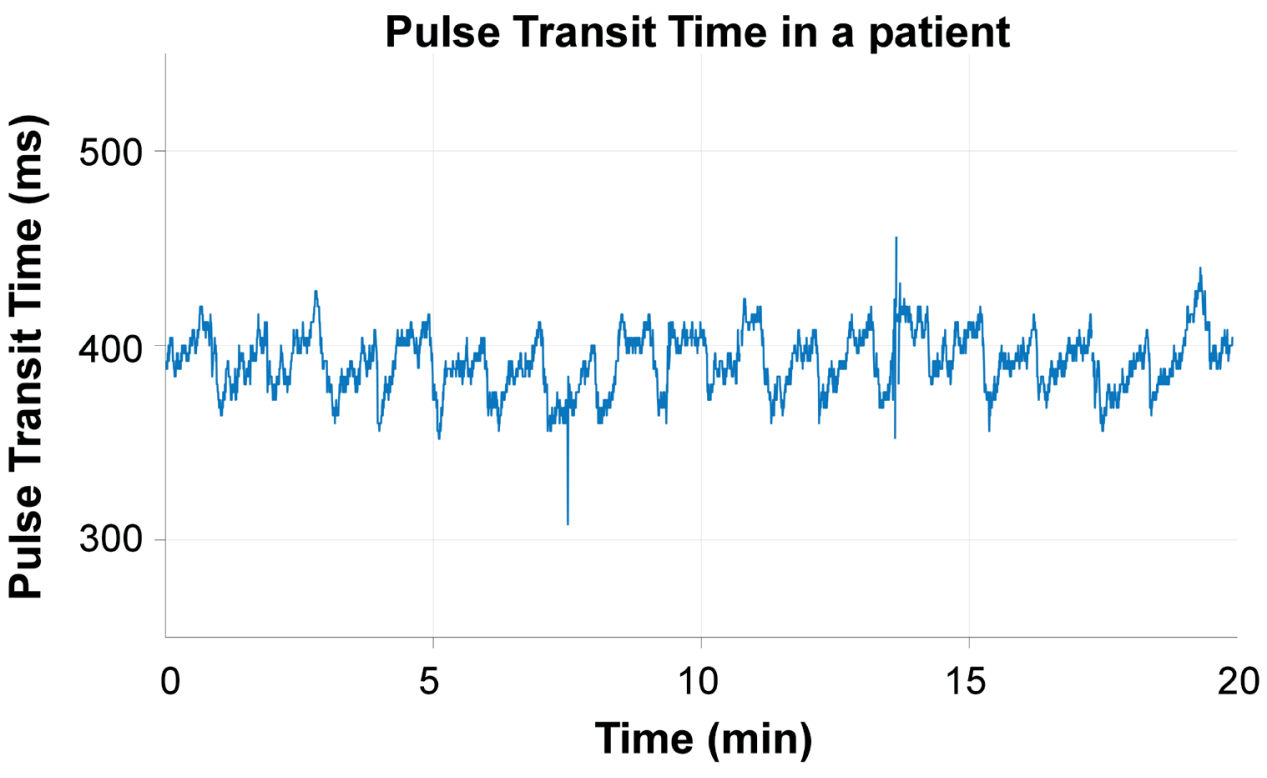

Figure 2.1: Clinically derived PTT of a preterm infant. A regular sawtooth-like artifact is seen, hampering clinical usefulness. 


\section{$2.2 \quad$ Methods}

\subsubsection{Simulation design}

Experiments were performed at the neonatal intensive care unit at the Máxima Medisch Centrum in Veldhoven, The Netherlands. The Vital Signs Simulator ProSim 8 (Fluke Biomedical, Cleveland, USA) was used to create an artificial analog ECG output and an optical signal changing in intensity mimicking blood flow. The optical PPG sensor used (Masimo SET SpO $\mathrm{O}_{2}$-technology, Irvine, USA) has a light-emitting end as well as a light-receiving end. The light-emitting end offers pulsed light signals that are modulated due to the presence of blood in the tissue between the light-emitting and light-receiving ends of the sensor. These modulated signals are subsequently received by the light-receiving end. The intensity and duration of the light pulses are under control of the PPG post-processing module. In our setup, the simulator modulated the pulsed light signal in amplitude thereby mimicking a light signal that has passed through a finger with a pulsating blood flow. For a flowchart of the setup we refer to Figure 2.2. The optical PPG sensor as well as the ECG output were in turn connected to a post-processing module (X2, Philips Medical Systems, Best, The Netherlands). The postprocessing module was connected to the patient monitor (IntelliVue MX80o, Philips Medical Systems, Best, The Netherlands), which in turn was connected via the central post of the neonatal intensive care unit to a central data warehouse (PIIC iX, Data Warehouse Connect, Philips Medical Systems, Best, The Netherlands), where ECG and PPG data were stored at 250 and $125 \mathrm{~Hz}$, respectively. The ECG and PPG were extracted for analysis from both the data warehouse and the monitor to test in which part of the chain potential artifacts occurred. Extracted data was stored on a personal computer (Microsoft Windows 1o Pro, HP EliteBook 850 G3, Palo Alto, USA). Simulations were performed at two different, but constant heart rates (100 and 160 beats per minute $(\mathrm{BPM})$ ) and processed by two different modules (X2 Ao3 and X2 AO5), resulting in a total of four simulations. To exclude that the simulator itself or the light pulses offered by the PPG sensor introduced the sawtooth phenomenon in the PTT, a photosensitive diode was placed at the light-receiving end of the PPG sensor. The signal from this diode was amplified and sampled together with the ECG from the simulator at $25000 \mathrm{~Hz}$ (Acquisition system IdeeQ, Instrumental Department Maastricht University, The Netherlands). The thus reconstructed PPG was smoothed over five samples to remove fluctuations. Estimation of PTT using these signals was done in the same manner as for the ECG and PPG from the data warehouse.

\subsubsection{PTT calculation}

Signal analysis was performed using MATLAB (2016A, The MathWorks, Natick, MA, USA). PTT was defined as the difference in time between the R peak in the ECG and the point where the increase in the upstroke of the PPG was $50 \%$ of the total increase (Figure 2.3). The R peak in the ECG was found using the MATLAB build-in function findpeaks. The point of $50 \%$ increase in the PPG was selected by using the findpeaks function to find the lower and upper peak of the upstroke, followed by the selection of the first point where the increase was equal to or higher than $50 \%$. The difference between the time instances for each ECG peak and subsequent PPG peak were calculated in milliseconds. 


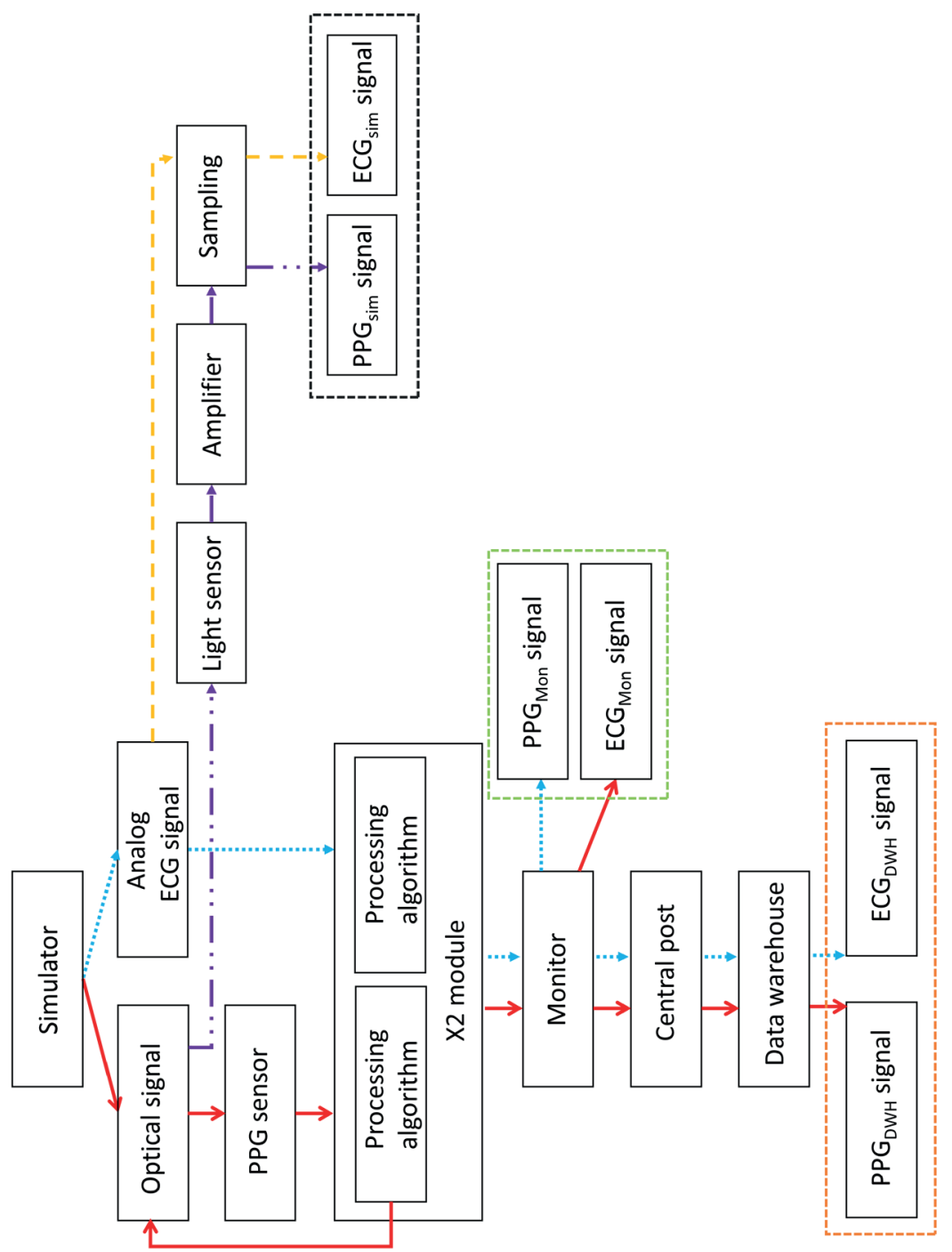

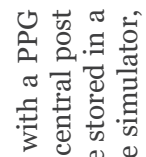

卷导

范

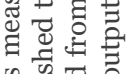

乌ิ

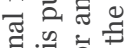

.

뜌

若. 总

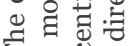

सं छ

范范

के च्च 명

.

०.ज

สี 㢳

สี

ల.

$\infty 0$ 추

䒕

สิ 000 तै

สี พี

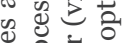

ฮี

琎

후 잏

넝

范要

का

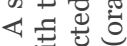

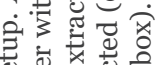

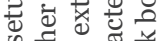

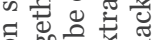

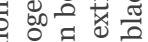

뜰웡

急

प्व

视志家

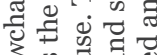

उ.

工 웡

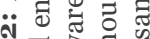

ง

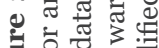

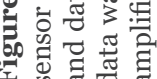




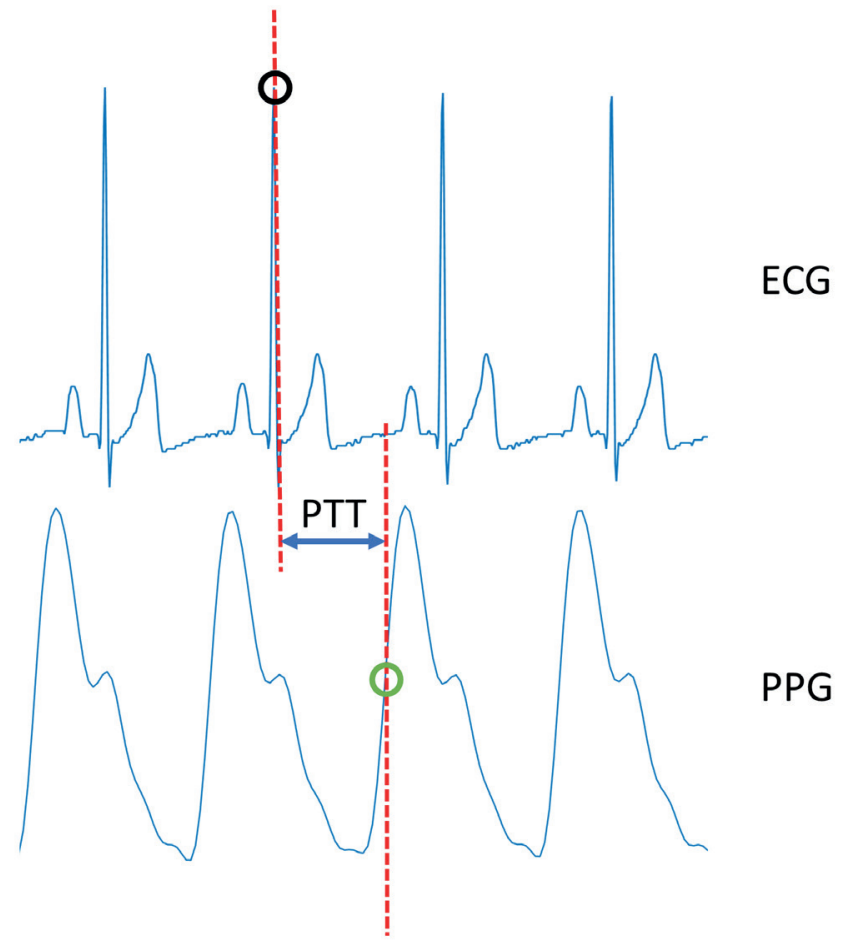

Figure 2.3: Illustration of pulse transit time calculation. Pulse transit time is depicted as the time difference between the R peak in the ECG (black circle) and the point of 50\% increase in the PPG signal (green circle).

\subsection{Results}

The PTT calculated for the Ao3 module (100 BPM signal in orange and 160 BPM in red) and the Ao5 module (100 BPM in black and 160 BPM in blue) are shown in Figure 2.4A. A recurring sawtooth-like phenomenon was detected in all simulations. The average length, slope and mean amplitude of the sawtooths are shown in Table 2.1 for all four simulations.

\begin{tabular}{|lcccc|} 
& \multicolumn{2}{c}{ A03 } & \multicolumn{2}{c|}{ Ao5 } \\
& 100 BPM & 160 BPM & 100 BPM & 160 BPM \\
\hline Length (s) & 60.8 & 59.2 & 72.4 & 72.4 \\
\hline Slope (ms/min) & 35.6 & 34.4 & 25.9 & 25.5 \\
\hline Mean amplitude (ms) & 36.0 & 33.9 & 31.3 & 30.8 \\
\hline
\end{tabular}

Table 2.1: The length, slope and mean amplitude of an average sawtooth are shown for different heart rates (100 BPM and 160 BPM) with different post-processing modules (Ao3 and Ao5). 

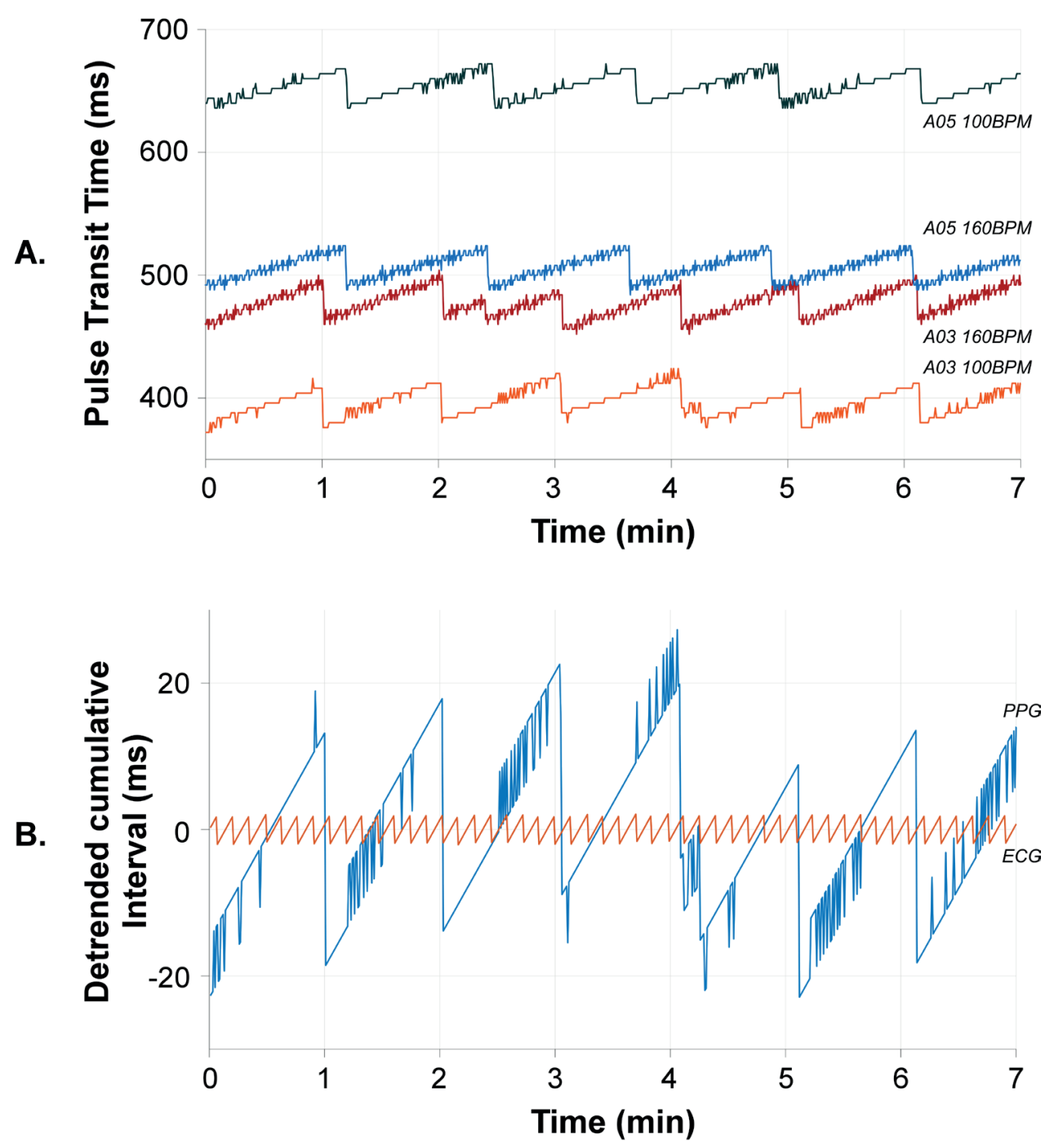

Figure 2.4: Pulse transit time and analysis for multiple simulations. A. Pulse transit time over several minutes for all four combinations of post-processing modules (Ao3 and A05) and heart rate (100 BPM and 160 BPM) using the Masimo oximeter. A clear sawtooth was observed in all simulations. B. Detrended cumulative intervals for PPG and ECG using the Ao3 module with 100 BPM. PPG shows a clear sawtooth pattern. The small fluctuations in ECG and PPG correspond to a single sample. 
As the signal is simulated at a constant heart rate, the interval between subsequent $\mathrm{R}$ peaks or subsequent PPG peaks should remain equal. Therefore, the cumulative sum of these intervals over time should result in a straight upward line. Detrending this cumulative sum should result in a constant value of zero in case of a constant heart rate and stroke volume. The detrended cumulative intervals of the PPG and the ECG using the Ao3 module at 100 BPM are shown in Figure 2.4B. The detrended cumulative ECG interval did not show a slope, indicating that on average, every ECG complex had an equal length. However, the detrended cumulative PPG interval resembled the sawtooth as found in the PTT. This indicates that each waveform of the PPG signal was slightly prolonged, corrected for by a periodic shortening of a single waveform. The amount of prolongation and correction relate to the slope and the amplitude of the sawtooth in the PTT, respectively.

At similar heart rates but with different post-processing modules, PTT varied up to $250 \mathrm{~ms}$, indicating a post-processing module dependent shift of the ECG and/or the PPG in time.

The PTT calculated from data directly extracted from the simulator did not show a sawtooth and was not influenced by heart rate or the module used to provide the optical signal (Figure 2.5). Because data extracted from the monitor and the data warehouse showed exactly the same sawtooth-like artifact, we conclude that the sawtooth artefact has its origin between the simulator and the monitor, i.e. it occurs in the post-processing module. This conclusion is supported by the module-dependent changes in the sawtooth characteristics.

\section{Pulse Transit Time using simulated ECG and PPG}

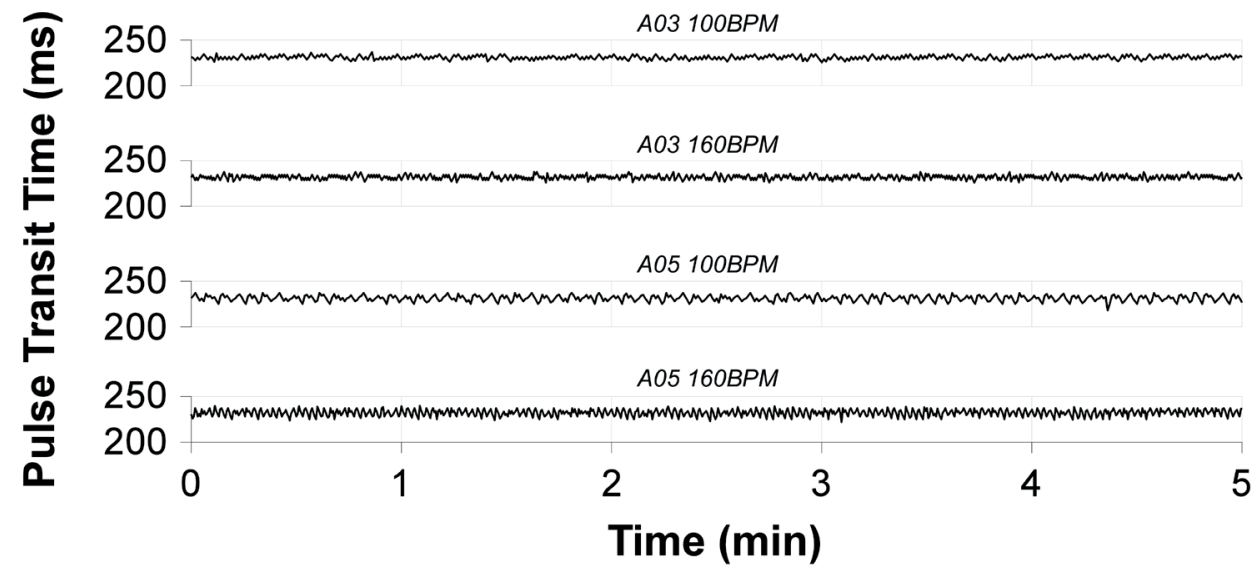

Figure 2.5: Pulse transit time as calculated from data extracted directly from the simulator. Pulse transit time over several minutes for all four combinations of postprocessing modules (Ao3 and A05) and heart rate (100 BPM and 160 BPM) using direct extraction out of the simulator. No large sawtooth is observed. Small fluctuations are caused by shifting of detected peaks with one sample. 


\subsection{Discussion}

We observed in our clinical monitor system a sawtooth pattern in the PTT, which has its origin in the Masimo post-processing module of the PPG signal in the Philips patient monitor. The detected sawtooth demonstrates that clinical patient monitoring systems need to be tested before they can be used for PTT measurements. Although direct export of the simulation signal did not show a sawtooth, this artifact was observed in the signal acquired directly at the patient monitor. Therefore, the sawtooth artifact is not a result of delays in signal transport between the patient monitor and the central post. Characteristics of this sawtooth were independent of the heart rate. However, the duration and slope of the sawtooth changed with different post-processing modules. This confirms that the sawtooth is generated in the postprocessing module. Inspection of the individual ECG and PPG signals showed that the ECG did not show alterations in time, while all PPG waveforms were slightly prolonged, corrected for by a periodic shortening of a single waveform. Therefore, we conclude that postprocessing of the PPG waveform induces the sawtooth artifact. In addition to the sawtooth artifact, the change in PTT using different post-processing modules indicates that the ECG and/or the PPG are also shifted in time with a non-equal time interval. The results found in our study are in line with earlier observations by Foo et al., which describe a high variation in PTT using a Masimo oximeter [13]. Our study proves that the variation observed is actually a periodic artifact combined with a phase delay, both due to post-processing in the Masimo module.

We observed that the PTT ${ }_{\text {sim }}$ was constant over time and independent of HR and the postprocessing module. However, the $\mathrm{PTT}_{\mathrm{DWH}}$ showed a sawtooth with amplitude up to $36.0 \mathrm{~ms}$ and a shift in PTT values up to $250 \mathrm{~ms}$, dependent on HR and the post-processing module used. Amirtharaj et al. found that the mean PTT for preterm infants with a closed ductus arteriosus is as small as $65.5 \mathrm{~ms}$ [11]. Even though in this study the PTT has a resolution of $8 \mathrm{~ms}$, it is clear that artifacts as large as measured in the current setup result in a PTT that cannot be used as an absolute value in clinical situations and thus not as a diagnostic tool. The finding that the PTT is unreliable as a diagnostic tool has to be taken into account in current research, as current research focusses on PTT as a predictive or trend parameter [10,14,15]. Our study emphasizes the importance of quality control in the setup used for measuring PTT.

To enable the use of PTT in a clinical setting, the cause of the phase shift and the sawtooth should be identified and removed by the manufacturer. An alternative is calibration of the signal based on a known input. We observed that the shift in PTT time and the sawtooth characteristics differ per post-processing module. As the shift in time is HR dependent and HR is not constant in patients, it is not possible to correct for this shift in time without knowing the exact nature of the dependencies. When the shift in time of the signals remains unknown, the absolute value of the PTT has no meaning. Therefore, the only clinical application of PTT is the use as a trend monitor. To use the PTT as a trend monitor, the sawtooth has to be corrected by a moving averaging filter with a window that spans the sawtooth artifact. This application is not able to detect beat-to-beat differences or to compare a patient to a reference value, as is necessary for predictive purposes. Therefore, for clinical applications of the PTT it is of utmost importance that both phase shift and sawtooth are prevented from occurring in the post-processing module. 
Our study has several limitations. First, though we demonstrated that the Masimo postprocessing module is responsible for the occurrence of the sawtooth, we cannot indicate why these artifacts occur because the algorithms used for post-processing are unknown to us. Therefore, we cannot provide solutions, but only cumbersome workarounds to use the current data to the fullest extent. Second, our methods to detect alterations in the time domain depend on raw waveform information, which is not always provided. Based on our findings, it is necessary to control if a sawtooth and a phase delay are present in the PTT. Therefore, systems that output the PTT without providing PPG- and ECG-signals should not be used.

\subsection{Conclusion}

We demonstrated that post-processing of the PPG signal in the Masimo module of the Philips patient monitor introduces a sawtooth in the PPG and in the derived PTT. This sawtooth, together with a large module-dependent phase shift, renders the PTT insufficient for clinical purposes. Whether these artifacts are vendor-specific is unknown. We conclude that before using time-dependent parameters like PTT for clinical purposes, the monitoring system needs to be checked on its accuracy. 


\subsection{References}

1. Elgendi M. On the Analysis of Fingertip Photoplethysmogram Signals. Curr Cardiol Rev. 2012;8(1):14-25.

2. Allen J. Photoplethysmography and its application in clinical physiological measurement. Physiol Meas. 2007;28(3):1-39.

3. Foo JYA, Wilson SJ. Clinical applications of pulse transit time in paediatric critical care. J Med Eng Technol. 2009;33(1):79-86.

4. Kawagoe Y, Sameshima H, Ikenoue T. Clinical application of pulse transit time and correlation with intrapartum fetal heart rate monitoring: A preliminary study of 18 fullterm infants. Reprod Sci. 2008;15(6):567-571.

5. Vlahandonis A, Biggs SN, Nixon GM, Davey MJ, Walter LM, Horne RSC. Pulse transit time as a surrogate measure of changes in systolic arterial pressure in children during sleep. J Sleep Res. 2014;23(4):406-413.

6. Kortekaas MC, Niehof SP, Van Velzen MHN, Galvin EM, Stolker RJ, Huygen FJPM. Comparison of bilateral pulse arrival time before and after induced vasodilation by axillary block. Physiol Meas. 2012;33(12):1993-2002.

7. Foo JYA, Wilson SJ, Williams G, Harris MA, Cooper D. Age-related factors that confound peripheral pulse timing characteristics in Caucasian children. J Hum Hypertens. 2005;19(6):463-466.

8. Babchenko A, Davidson E, Adler D, Ginosar Y, Kurz V, Nitzan M. Increase in pulse transit time to the foot after epidural anaesthesia treatment. Med Biol Eng Comput. 2000;38(6):674-679.

9. Chakrabarti B, Emegbo S, Craig S, Duffy N, O’Reilly J. Pulse transit time changes in subjects exhibiting sleep disordered breathing. Respir Med. 2017;122:18-22.

10. Smith LA, Dawes PJ, Galland BC. The use of pulse transit time in pediatric sleep studies: A systematic review. Sleep Med Rev. 2018;37:4-13.

11. Amirtharaj CR, Palmeri LC, Gradwohl G, Adar Y, Nitzan M, Gruber D, et al. Photoplethysmographic assessment of pulse transit time correlates with echocardiographic measurement of stroke volume in preterm infants with patent ductus arteriosus. J Perinatol. 2018;38(9):1220-1226.

12. Goudjil S, Imestouren F, Armougon A, Razafimanantsoa L, Mahmoudzadeh M, Wallois F, et al. Noninvasive Technique for the Diagnosis of Patent Ductus Arteriosus in Premature Infants by Analyzing Pulse Wave Phases on Photoplethysmography Signals Measured in the Right Hand and the Left Foot. PLoS One. 2014;9(6):e98763.

13. Foo JYA, Wilson SJ, Dakin C, Williams G, Harris M-A, Cooper D. Variability in time delay between two models of pulse oximeters for deriving the photoplethysmographic signals. Physiol Meas. 2005;26(4):531-544.

14. Kim YU, Kim DH, Cheong Y, Kong Y, Lee J, Park SK, et al. Pulse Transit Time as a Predictor of the Efficacy of a Celiac Plexus Block in Patients With Chronic Intractable Abdominal Pain. Clin J Pain. 2016;32(6):522-526.

15. Escobar-Restrepo B, Torres-Villa R, Kyriacou PA. Evaluation of the Linear Relationship Between Pulse Arrival Time and Blood Pressure in ICU Patients: Potential and Limitations. Front Physiol. 2018;9:1848. 



\section{Chapter 3}

Ratio of arterial blood pressures at borders of window surrounding systolic peak indicates patent ductus arteriosus in preterm infants

Frank C. Bennis, Peter Andriessen, Carola van Pul, Boris W. Kramer, Tammo Delhaas

In preparation 


\begin{abstract}

\section{Background}

Presence of a patent ductus arteriosus (PDA) in neonates is assessed by echocardiography. Echocardiographic assessment has disadvantages, primarily its discontinuous nature. We hypothesize that the continuously measured ratio of arterial blood pressures (ABP) at the borders of a window surrounding the systolic peak ratio discriminates non-PDA from PDA patients.
\end{abstract}

\title{
Methods
}

Preterm infants (gestational age $<32$ weeks) with and without PDA were included. Patients were divided into controls $(n=8)$, PDA patients $(n=22)$, and patients with closed PDA after three doses Ibuprofen $(n=10)$. For each patient, a six-hour ABP segment from 12 AM to 6 $\mathrm{AM}$ on the day of echocardiography assessing patency or closure of the DA was selected. The mean ratio of the $A B P$ values a samples before and p samples after the systolic peak $\left(R_{A B P}\right)$ was calculated for each segment. If $\mathrm{R}_{\mathrm{ABP}}<1$, the patient was predicted to have a PDA. The a and $\mathrm{p}$ with the least misclassifications were selected (-64 and $+104 \mathrm{~ms})$.

\section{Results}

$\mathrm{R}_{\mathrm{ABP}}$ was significantly lower in PDA patients (median 0.95, IQR 0.06) compared to controls (median 1.05, IQR 0.10; $\mathrm{p}=0.0024) . \mathrm{R}_{\mathrm{ABP}}$ correctly predicted 19 out of 22 patients $(86.4 \%$ ) and 6 out of 8 controls (75\%). $\mathrm{R}_{\mathrm{ABP}}$ increased after closure in 9 out of 10 patients (median 1.01, IQR 0.04; $\mathrm{p}=0.0182$ ).

\section{Conclusion}

$\mathrm{R}_{\mathrm{ABP}}$ can discriminate preterm PDA patients from non-PDA patients and can be calculated continuously from clinical data measured during standard of care. 


\subsection{Introduction}

Closure of the ductus arteriosus (DA) is necessary in postnatal circulation as it ensures a serial circulation with blood first passing through the lungs for oxygenation and subsequently through the body. Failure of closing the DA results in a patent DA (PDA). A PDA in the postnatal circulation may result in a hemodynamically significant left-to-right shunt with blood flowing from the aorta to the pulmonary circulation. This pattern of blood flow may lead to systemic hypoperfusion and pulmonary hypertension and is associated with major neonatal morbidities involving the gut, brain and lungs as well as with an increase in overall mortality [1-4]. In very low birth weight infants (below $1000 \mathrm{~g}$ ), PDA is present in $39 \%$ to $66 \%[5,6]$. This high incidence and the severe comorbidities of a hemodynamically significant PDA suggest the importance of timely detection and treatment of a hemodynamically significant PDA.

Though echocardiography is routinely performed to assess ductal patency a couple of days after birth, it has several disadvantages: 1) Ductal patency is assessed at a single discrete moment in time, whilst ductal patency and hemodynamic consequences may differ over time: a PDA may close spontaneously, but a non-significant PDA may also progress to a hemodynamically significant PDA [5,7,8]. 2) Echocardiography, a time- and recoursesconsuming procedure, has to be performed by a pediatric cardiologist or a well-trained neonatologist [9], who may not be available at any moment.

We therefore aim to identify a continuously measured parameter that can discriminate a PDA from a closed DA. This parameter should be based on clinical data obtained during standard of care. One such parameter may be extracted from the arterial blood pressure (ABP) waveform, as it has been shown that the ABP waveform is influenced by a PDA [10-13]. Blood flow from the aorta through the PDA to the pulmonary artery results in a faster decline of the ABP during diastole [12,13]. Furthermore, the PDA can be seen as a parallel artery, which may result in a slower build-up of pressure during systole (Figure 3.1). Therefore, we hypothesize that the ratio between the downward - and upward slope arterial blood pressure at the borders of a given window surrounding the systolic peak can discriminate patients with a PDA from patients with a closed DA. 


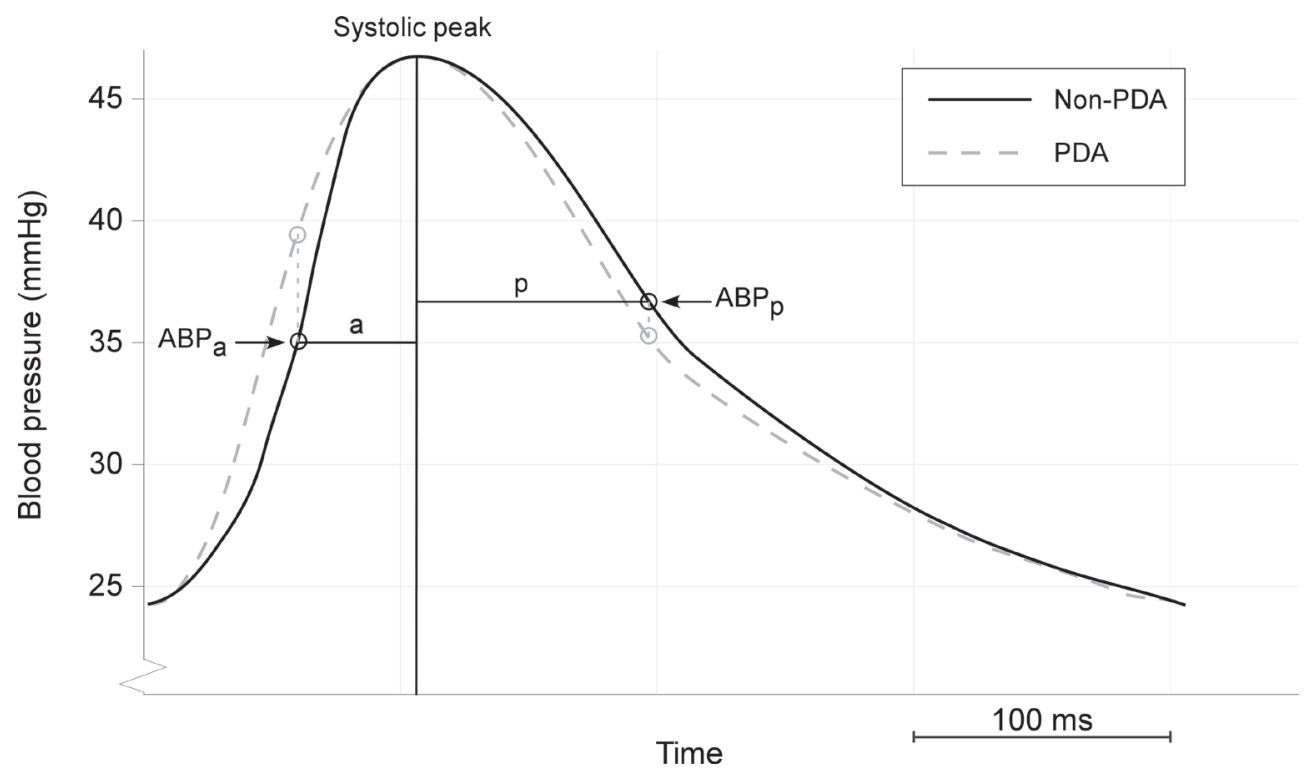

Figure 3.1: Hypothetical waveforms in patients without and with a PDA. Arterial blood pressure (ABP) is selected $\mathrm{p}$ samples after and a before the systolic peak. For PDA patients, $\mathrm{ABP}_{\mathrm{a}}$ is lower (gray dotted line) compared to non-PDA patients, while $\mathrm{ABP}_{\mathrm{p}}$ (gray dotted line) is higher for PDA patients.

\subsection{Methods}

\subsubsection{Design and subjects}

The patient population consisted of preterm infants without a PDA (controls) and of preterm infants with a PDA requiring treatment. All infants received standard of care at the neonatal intensive care unit (NICU) at the Máxima Medical Centre Veldhoven and were admitted between June 2016 and January 2019. The main inclusion criterium for the PDA group was a PDA that because of its hemodynamic significance was treated with a three day course of Ibuprofen. As controls served patients in which a closed ductus arteriosus was confirmed by echocardiography. Further inclusion criteria were a gestational age below or equal to 32 weeks, the first echo within two weeks after birth and invasive continuous ABP measurements from $12 \mathrm{AM}$ (midnight) of the day on which the first echo was performed until at least the time of the first echo. Exclusion criteria were treatment with inotropic drugs and clinical conditions related to overt patient instability, such as septic shock (positive blood culture), pneumothorax, intraventricular hemorrhage grade 3 or higher or necrotizing enterocolitis. As this was a retrospective study in which anonymized data corresponding to routine patient monitoring was used, the medical ethical committee provided a waiver in accordance with the Dutch law on medical research with humans. Note that none of the arterial catheters had to be introduced to the infants for this study; all used arterial catheters were part of standard of care. 


\subsubsection{Data collection}

Gestational age, birth weight, gender, location of the arterial line, ABP waveforms, patency of the DA, date and dose of Ibuprofen as well as date of echocardiographic examinations were stored for all included patients. Blood pressure waveforms were measured continuously by a 3.5 French invasive arterial line via the umbilical artery or a 22 Gauge catheter in the radial artery and stored at $125 \mathrm{~Hz}$ in a local data warehouse (PIIC iX, Data Warehouse Connect, Philips Medical Systems). Blood pressure waveforms were exported from this data warehouse to a personal computer (Microsoft Windows 10 Pro, HP EliteBook 850 G3) on which further analysis was done using MATLAB (2018A, The MathWorks, Natick, MA, USA). Blood pressure waveforms were selected from the start of blood pressure measurements up to the echo confirming closure of the DA, if available.

Subjects were divided into three groups: 1) controls 2) all PDA patients 3) PDA patients in whom ABP signals were available until echocardiography confirmed closure after three doses of Ibuprofen, which is thus a subset of group 2. Group one and two were used to evaluate the ability of a parameter to discriminate a patient with a PDA from a patient with a closed DA. Group two and three were used to evaluate the ability of the same parameter to indicate closure of a PDA after treatment. A six-hour ABP period (subsequently referred to as one segment) ranging from $12 \mathrm{AM}$ midnight to $6 \mathrm{AM}$ on the day of the first echo assessing patency of the DA was selected for each subject. We chose to analyze ABP only in the segment between $12 \mathrm{AM}$ midnight and $6 \mathrm{AM}$ to prevent variation between patients induced by time of the day. In group three, an additional six-hour ABP segment was measured from $12 \mathrm{AM}$ midnight to $6 \mathrm{AM}$ on the day the second echocardiography confirmed closure of the DA.

\subsubsection{Pre-processing}

Blood pressure waveforms were corrected for gain and offset. Diastolic minima and systolic peaks were selected using the findpeaks algorithm in MATLAB, corresponding to the downward and upward peaks, respectively. The minimum distance between diastolic values was set as $0.24 \mathrm{~s}$, equal to a maximal heart rate of $250 \mathrm{bpm}$, to prevent local peaks and minima to be selected as a systolic peak or diastolic minimum. To ensure that each diastolic minimum was followed by a systolic peak and vice versa, data points were excluded if two subsequent peaks were both in diastolic phase or both in systolic phase. The ABP signal was subsequently segmented into separate beats, each beat ranging from diastolic to diastolic minimum. After beat segmentation, artifacts were selected and removed on a beat-to-beat basis using a custom-made algorithm (Supplementary material 3.7.1). If more than $10 \%$ of all waveforms in the selected six-hour segment consisted of artifacts, the subject was excluded for analysis as the signal was considered too unstable in general. 


\subsubsection{Parameter calculation}

For each waveform in the artifact-free six-hour $\mathrm{ABP}$ segment, the $\mathrm{ABP}$ value $\mathrm{p}$ ms after $\left(\mathrm{ABP}_{\mathrm{p}}\right)$ and a ms before $\left(\mathrm{ABP}_{\mathrm{a}}\right.$ ) the systolic peak was selected (Figure 3.1). The ratio $\mathrm{ABP}_{\mathrm{p}} / \mathrm{ABP}_{\mathrm{a}}$, which is thus the ABP at the downward (posterior) slope divided by the $\mathrm{ABP}$ at the upward (anterior) slope, was calculated for each waveform. The mean over all ratios in the six-hour segment was subsequently determined (we call this $\mathrm{R}_{\mathrm{ABP}}$ ). An $\mathrm{R}_{\mathrm{ABP}}$ higher than 1 indicates that the ABP level p samples after the systolic peak is in general higher than the ABP level a samples before the systolic peak.

\subsubsection{Statistical analysis}

The time period after (post) and before (ante) the systolic peak (p and a) was varied in steps of $8 \mathrm{~ms}$ from 8 to $160 \mathrm{~ms}$ for $\mathrm{p}$ and -8 to $-80 \mathrm{~ms}$ for a, respectively. Assuming that $\mathrm{R}_{\mathrm{ABP}}<1$ indicates the presence of a PDA, the number of patients classified incorrectly was calculated for every combination of $\mathrm{p}$ and $\mathrm{a}$. The combinations with the lowest number of incorrectly classified patients were defined as optimal. If multiple combinations were selected as optimal, the combinations with the lowest number of incorrectly classified control patients were defined optimal. If still multiple combinations were present, the combination with the lowest p-value (Students T-test, controls vs all PDA patients) was selected as optimal. A paired T-test was performed to test for a change in $\mathrm{R}_{\mathrm{ABP}}$ in patients with a PDA before and after closure (group three) using the previously selected optimal values for $\mathrm{p}$ and $\mathrm{a}$.

\subsection{Results}

In the study period 36 patients were eligible for inclusion. Six out of these 36 patients were excluded because more than $10 \%$ of the waveforms in the analyzed time segment contained artifacts. The remaining 30 patients consisted of 8 controls (group one, 3 male) and 22 patients with a PDA (group two, 10 male) (Table 3.1). Ten out of the 22 patients with a PDA showed closure of the DA after three doses of Ibuprofen and had ABP measurements during the whole treatment period (group three, 3 male). In 20 infants ABP was measured via the umbilical artery with the tip of the catheter just above the diaphragm. In 10 infants ABP was measured via a catheter in the radial artery.

\subsubsection{Optimal window selection}

The total amount of misclassified patients $\left(\mathrm{R}_{\mathrm{ABP}}<1\right.$ is classified as a PDA patient) was calculated for every combination of $\mathrm{p}$ and $\mathrm{a}$. A surface plot of the number of misclassifications for each combination of $\mathrm{p}$ and $\mathrm{a}$ is given in Figure 3.2. With increasing $\mathrm{p}$, a should also increase to obtain a significant p-value, showing a diagonal line in Figure 3.2. If a is taken too small compared to $\mathrm{p}$, the ABP level is selected too close to the systolic peak and is therefore almost always lower at the end than at the begin of the selected window, resulting in low $R_{A B P}$ for every patient. The opposite is true if $\mathrm{p}$ is taken too small compared to a. The optimal $\mathrm{p}$ and a are found to be +104 and $-64 \mathrm{~ms}$, respectively, with five misclassified subjects, of which two are control subjects. 


\subsubsection{Detection of a PDA before the first echocardiography}

The $\mathrm{R}_{\mathrm{ABP}}$ of patients with a closed DA (group one, median 1.05, IQR 0.10) was significantly higher than the $\mathrm{R}_{\mathrm{ABP}}$ of PDA patients (group two, median 0.95, IQR 0.06; $\mathrm{p}=0.0024$ ) (Figure 3.3, first vs. second boxplot). Two PDA patients (both with an umbilical catheter) showed a remarkable high $\mathrm{R}_{\mathrm{ABP}}$. However, patient data and $\mathrm{ABP}$ data did not provide any reason to exclude those patients. In the current outcome, 19 out of 22 PDA patients (86.4\%) and 6 out of 8 control patients (75\%) are classified correctly using an $\mathrm{R}_{\mathrm{ABP}}$ cutoff value of 1 . We did not find a correlation between heart rate and $\mathrm{R}_{\mathrm{ABP}}$ (data not shown).

\subsubsection{Evaluation of PDA closure after Ibuprofen treatment}

The $\mathrm{R}_{\mathrm{ABP}}$ of PDA patients in group 2 on the day of the first echocardiography confirming a PDA was compared to the $\mathrm{R}_{\mathrm{ABP}}$ within the same patients on the day of the second echocardiography confirming closure of the DA. A significant increase in $\mathrm{R}_{\mathrm{ABP}}$ after PDA closure was observed (median 1.01, IQR 0.04, $\mathrm{p}=0.0182$, Figure 3.3, second vs. third boxplot). $\mathrm{R}_{\mathrm{ABP}}$ increased in 9 out of 10 patients. No reason was found to exclude the single patient in whom $\mathrm{R}_{\mathrm{ABP}}$ decreased. $\mathrm{R}_{\mathrm{ABP}}$ after closure lies in 6 out of 10 patients above 1 .

\begin{tabular}{|c|c|c|c|c|}
\hline & $\begin{array}{l}\text { Controls } \\
(n=8)\end{array}$ & $\begin{array}{l}\text { All PDA } \\
\text { patients } \\
(\mathbf{n}=\mathbf{2 2})\end{array}$ & $\begin{array}{l}\text { Patients with } \\
\text { closure of PDA } \\
(n=10)\end{array}$ & P-value \\
\hline $\begin{array}{l}\text { Gestational age (weeks + } \\
\text { days, (median (IQR)) }\end{array}$ & $\begin{array}{l}26+2.5(24+2 \\
-28+2.5)\end{array}$ & $\begin{array}{l}26+1(25+6 \\
-26+4)\end{array}$ & $\begin{array}{l}26+1(25+6- \\
26+4)\end{array}$ & 0.58 \\
\hline Birth weight (g, mean (SD)) & $854(256)$ & $855(150)$ & $777(147)$ & 0.48 \\
\hline Male (n (\%)) & $3(37.5)$ & $10(45)$ & $3(30)$ & 0.70 \\
\hline Umbilical arterial line (n, \%) & $4(50)$ & $16(72,7)$ & $6(60)$ & 0.48 \\
\hline $\begin{array}{l}\text { Mean arterial pressure over } \\
\text { six-hour segment }(\mathrm{mmHg} \text {, } \\
\text { mean (SD)) }\end{array}$ & $40.1(4.4)$ & $36.3(4.7)$ & $35.0(4.2)$ & 0.06 \\
\hline $\begin{array}{l}\text { Mean systolic arterial } \\
\text { pressure over six-hour } \\
\text { segment (mmHg, mean (SD)) }\end{array}$ & $54.8(8.8)$ & $49.9(6.1)$ & $49.2(7.0)$ & 0.18 \\
\hline $\begin{array}{l}\text { Mean diastolic arterial } \\
\text { pressure over six-hour } \\
\text { segment (mmHg, mean (SD)) }\end{array}$ & $29.9(3.0)$ & $26.5(4.6)$ & $25.1(3.6)$ & 0.048 \\
\hline $\begin{array}{l}\text { Mean heart rate over six- } \\
\text { hour segment (BPM, mean } \\
\text { (SD)) }\end{array}$ & $151.8(10.2)$ & $152.1(10.7)$ & $149.4(9.1)$ & 0.78 \\
\hline
\end{tabular}

Table 3.1: Demographics and hemodynamic variables of the patients used. Demographics are split into controls (group 1), all PDA patients (group 2) and patients in whom the PDA closed after Ibuprofen treatment (group 3). P values are calculated by a Chi-Square test or a one-way-Anova. 


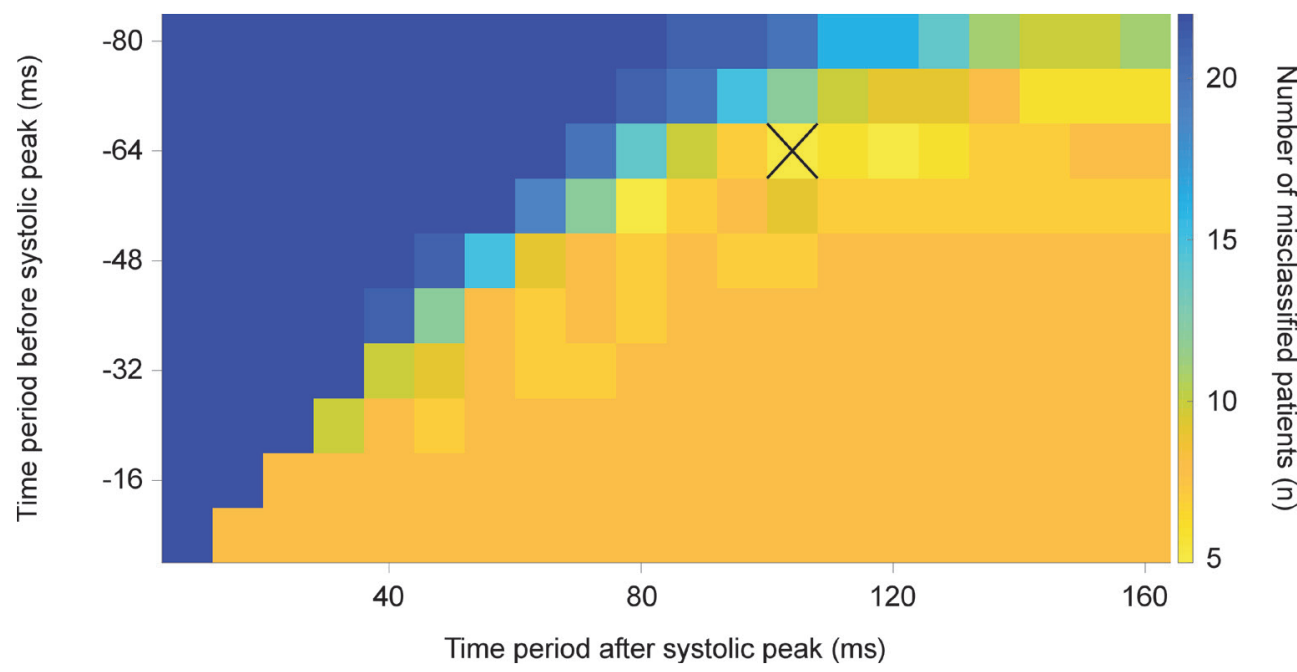

Figure 3.2: Surface plot showing the number of misclassified patients using arterial blood pressure ratios for each possible window (combination of the time period before and time period after the systolic peak). With an increasing time period after the systolic peak, the time period before the peak also has to increase to obtain significant differences. The optimal window is $64 \mathrm{~ms}$ before to $104 \mathrm{~ms}$ after the systolic peak (black cross).

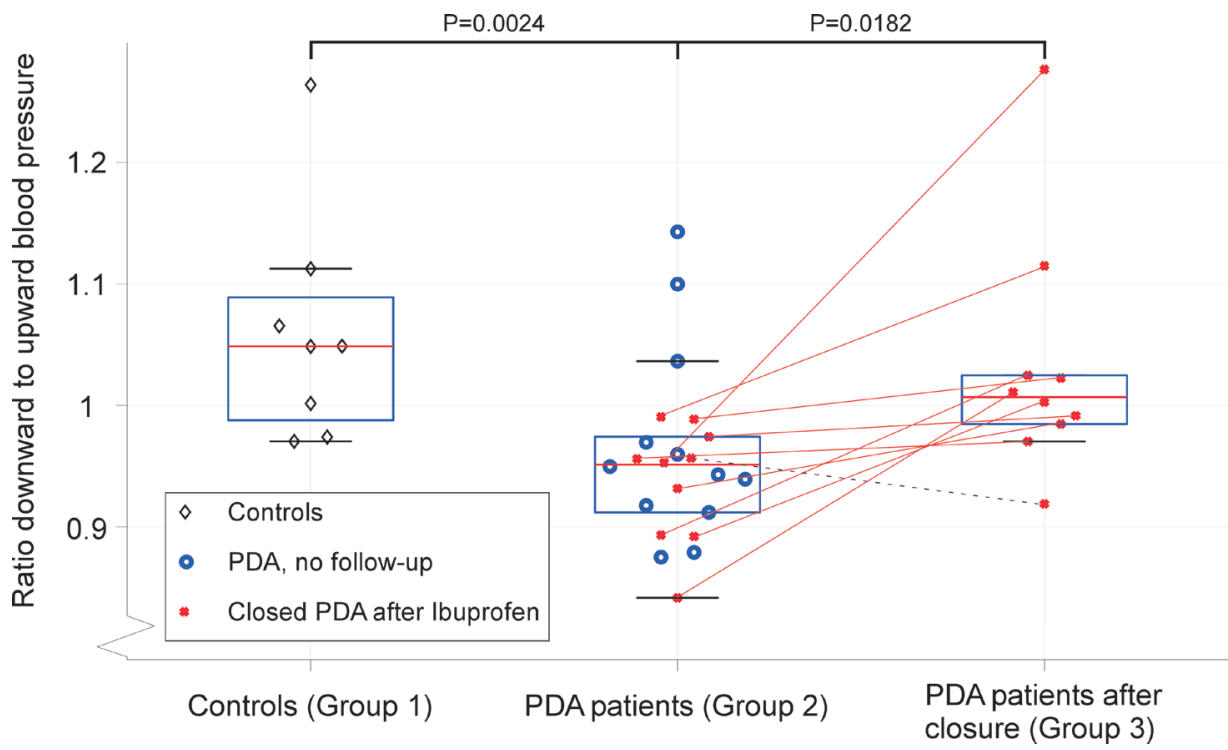

Figure 3.3: $\mathrm{R}_{\mathrm{ABP}}$, i.e. the ratio of $\mathrm{ABP}_{\mathrm{p}}$ (104 ms after the systolic peak) divided by $\mathrm{ABP}_{\mathrm{a}}$ ( $64 \mathrm{~ms}$ before the systolic peak) for controls ( $n=8,3$ male), all PDA patients $(n=22,10$ male) and a subset of PDA patients that showed a closed ductus after buprofen treatment ( $n=10,3$ male)). A significant lower $R_{A B P}$ is seen for patients where a PDA is present compared to controls as tested by the Students T-test. A significant increase in the ratio is observed after closure of the PDA as tested by a paired T-test. Red lines indicate an increase in $\mathrm{R}_{\mathrm{ABP}}$ after closure, the black dotted line indicates a decrease. 


\subsection{Discussion}

This study showed that the ratio between the down- and upward slope ABP pressure at the borders of a given window surrounding the systolic peak can discriminate a patient with a PDA from a patient with a closed DA with a correct classification rate of $83.3 \%$. Furthermore, $\mathrm{R}_{\mathrm{ABP}}$ significantly increased after closure in patients with a PDA. Because ABP can be assessed continuously shortly after birth and is based on clinical data measured during standard of care, this study indicates that $\mathrm{R}_{\mathrm{ABP}}$ is useful to assess ductal patency at a single point in time as well as ductal patency after Ibuprofen treatment.

The lower $\mathrm{R}_{\mathrm{ABP}}$ in patients with a PDA was due to a smaller increase in ABP before the systolic peak and a larger decrease in $\mathrm{ABP}$ after the systolic peak compared to controls. The larger decrease in ABP after the systolic peak in patients with a PDA may be explained by ductal steal, which accelerates the decrease in pressure during diastole and therefore lowers the diastolic blood pressure in the presence of a PDA $[12,14,15]$. The smaller increase in ABP in the time window before the systolic peak in patients with a PDA is harder to grasp. In theory, a PDA results in a higher preload of the left ventricle, resulting in increased contractility due to the Frank-Starling effect and therefore a larger increase in ABP [16]. However, it has been shown in preterm infants that although a higher preload leads to an increased ejection fraction, it does not lead to an increase in contractility [17]. Therefore, the total increase in stroke volume following a larger left ventricular end-diastolic volume is smaller in preterm infants than in term infants [18]. The combination of these notions may explain why the effect of an increased preload on the increase in ABP within the selected window is small. In addition, it is possible that ABP increases less steeply in PDA patients due to a larger combined crosssectional area of the systemic arteries. This may result in a broader distribution of the cardiac output, dampening the waveform and reducing the increase in ABP before the systolic peak. We expect a difference in heart rate to mostly affect the latter part of the diastolic phase and thus not the selected windows surrounding the systolic peak, which is in line with the findings that we did not find a correlation between heart rate and $\mathrm{R}_{\mathrm{ABP}}$.

Our finding that the ABP decreases more in the selected window after the systolic peak in patients with a PDA corresponds to literature $[12,13]$. Our finding that the increase in ABP in the selected window before the systolic peak is larger in patients without a PDA differs from literature. Gevers et al. found that PDA patients often show a pulsus bisferiens [11,19]. The initial peak of a pulsus bisferiens is significantly higher in patients with a PDA than in patients without a PDA and corresponds to a larger increase in ABP. However, our study did not find a pulsus bisferiens in most patients with a PDA. This may be because we used a fluid-filled catheter, whereas Gevers et al. used a tip-manometer. The fluid-filled catheter may dampen the waveform, canceling out the pulsus bisferiens. Interestingly, Gevers et al. did find a significantly shorter time period to systolic peak in patients without a PDA. In our study, the pulsus bisferiens was suppressed, which would make that a shorter time to systolic peak results in a larger increase in ABP for non-PDA patients compared to PDA patients since the same window length is selected. These findings suggest that the increase in ABP before the systolic peak may indeed be larger in a given time window for non-PDA patients compared to PDA patients. 
Currently, treatment of a PDA is under debate [20-22]. Some authors suggest to treat very conservatively or even keep a PDA untreated, as it may close on its own over time, thereby avoiding unnecessary treatment [23-26]. In this current study, an $\mathrm{R}_{\mathrm{ABP}}$ cutoff value of 1 was used, which led to a sensitivity of $86.4 \%$ and a specificity of $75 \%$. Using this cutoff value without additional echocardiography, three out of 22 PDA patients would not receive treatment, whilst two out of eight controls would receive treatment. The use of $\mathrm{R}_{\mathrm{ABP}}$ with a cutoff value of 1 may therefore accurately indicate the presence of a PDA. However, it is unlikely that such a measure will fully replace echocardiography for the detection of a PDA, since misclassifications still exist. A different approach is to shift focus from prediction of PDA to avoiding treatment in patients without a PDA. This shifted focus results in a $100 \%$ specificity rate while still detecting a PDA in 16 out of 22 patients. A more conservative way of implementation is to interpret $\mathrm{R}_{\mathrm{ABP}}$ as an indicator how likely a patient has a PDA. In this manner, the clinician can decide which patients should be screened for a PDA first. Although the choice depends on the clinician's preference, each method may reduce the number of echocardiographies needed. Since our method uses data that is already available and processes the data automatically up to the value of $\mathrm{R}_{\mathrm{ABP}}$, our method is an easy to use and costeffective addition to echocardiography. Another application of our method is early detection of a potential PDA, which can subsequently be confirmed by echocardiography and can thus be treated in an early phase. This possibility has to be evaluated further in a prospective study. Furthermore, our method classifies a high number of PDA patients correctly, enabling adequate treatment in this patient group.

A significant difference was found in the $\mathrm{R}_{\mathrm{ABP}}$ before closure of the PDA compared to the $\mathrm{R}_{\mathrm{ABP}}$ after closure of the PDA in the same patient. This indicates that $\mathrm{R}_{\mathrm{ABP}}$ may also be used to evaluate the progress of ductal closure over time. It has to be noted that the $\mathrm{R}_{\mathrm{ABP}}$ was still below 1 in four out of ten patients after ductal closure, indicating that focus has to be given to the progression of $\mathrm{R}_{\mathrm{ABP}}$ over time instead of the absolute value to evaluate ductal closure. Another possible application is the use of $\mathrm{R}_{\mathrm{ABP}}$ to observe progression of ductal closure shortly after birth. However, verification in a larger dataset is necessary to test the hypothesis that $\mathrm{R}_{\mathrm{ABP}}$ is indicative of ductal closure over time.

Our study has several limitations. First, ABP measurements via the umbilical artery were in general discontinued after the first week. The limited period of invasive ABP measurements constrained the possibility to follow-up PDA closure. Therefore, we were not able to include sufficient patients to correct the increase in $\mathrm{R}_{\mathrm{ABP}}$ after closure following treatment for possibly influencing factors such as increasing postnatal age. Second, this study defined the optimal window surrounding the systolic peak in the waveform to discriminate patients with and without a PDA in a retrospective dataset. However, the window is specific for this dataset and it is not known what the effect of gestational age is upon the optimal window length. Therefore, the outcome has to be validated in a larger independent dataset gathered during a prospective study and stratified according to gestational age. Third, although exclusion of severe comorbidities implied that none of our patients received any inotropic drug, medication other than Ibuprofen was not taken into account. It is possible that co-medication (e.g. caffeine) influenced contractility and therefore the speed of the upstroke. Since our study shows that the $\mathrm{R}_{\mathrm{ABP}}$ is a promising parameter to identify a PDA in preterm infants, the next step for further studies and analyses would be to include the sickest of patients and the 
influence of medication. Fourth, we included patients with umbilical as well as radial arterial lines. However, Gevers et al., have shown that the peripheral waveform resembles the central waveform in neonates [27]. Therefore, we do not expect the location of the arterial line to influence our results.

\subsection{Conclusion}

This study identified $\mathrm{R}_{\mathrm{ABP}}$, the ratio between $\mathrm{ABP}$ values at the borders of a given window surrounding the systolic peak, as a parameter able to discriminate preterm patients with a PDA from patients without a PDA. This ratio can be calculated from clinical data measured during standard of care. Furthermore, $\mathrm{R}_{\mathrm{ABP}}$ increased after closure of the PDA following treatment with Ibuprofen. We suggest validating $\mathrm{R}_{\mathrm{ABP}}$ in an external dataset over time with more patients in whom $\mathrm{R}_{\mathrm{ABP}}$ is measured prospectively and correlated to ductal closure and medication. 


\subsection{References}

1. Noori S, McCoy M, Friedlich P, Bright B, Gottipati V, Seri I, et al. Failure of Ductus Arteriosus Closure Is Associated With Increased Mortality in Preterm Infants. Pediatrics. 2009;123(1):e138-44.

2. Schena F, Francescato G, Cappelleri A, Picciolli I, Mayer A, Mosca F, et al. Association between Hemodynamically Significant Patent Ductus Arteriosus and Bronchopulmonary Dysplasia. J Pediatr. 2015;166(6):1488-92.

3. Evans N, Kluckow M. Early ductal shunting and intraventricular haemorrhage in ventilated preterm infants. Arch Dis Child Fetal Neonatal Ed. 1996;75(3):183-6.

4. Dollberg S, Lusky A, Reichman B. Patent Ductus Arteriosus, Indomethacin and Necrotizing Enterocolitis in Very Low Birth Weight Infants: A Population-Based Study. J Pediatr Gastroenterol Nutr. 2005;40(2):184-8.

5. Koch J, Hensley G, Roy L, Brown S, Ramaciotti C, Rosenfeld CR. Prevalence of spontaneous closure of the ductus arteriosus in neonates at a birth weight of 1000 grams or less. Pediatrics. 2006;117(4):1113-21.

6. Lemons JA, Bauer CR, Oh W, Korones SB, Papile L-A, Stoll BJ, et al. Very Low Birth Weight Outcomes of the National Institute of Child Health and Human Development Neonatal Research Network, January 1995 Through December 1996. Pediatrics. 2001;107(1):e1-e1.

7. Fink D, Nitzan I, Bin-Nun A, Mimouni F, Hammerman C. Ductus arteriosus outcome with focus on the initially patent but hemodynamically insignificant ductus in preterm neonates. J Perinatol. 2018;38(11):1526-31.

8. Semberova J, Sirc J, Miletin J, Kucera J, Berka I, Sebkova S, et al. Spontaneous Closure of Patent Ductus Arteriosus in Infants $\leq 1500$ g. Pediatrics. 2017;140(2):e20164258.

9. De Boode WP, Singh Y, Gupta S, Austin T, Bohlin K, Dempsey E, et al. Recommendations for neonatologist performed echocardiography in Europe: Consensus Statement endorsed by European Society for Paediatric Research (ESPR) and European Society for Neonatology (ESN). Pediatr Res. 2016;80(4):465-71.

10. Beuchée A, Pladys P, Senhadji L, Bétrémieux P, Carré F. Beat-to-beat blood pressure variability and patent ductus arteriosus in ventilated, premature infants. Pflügers Arch Eur J Physiol. 2003;446(2):154-60.

11. Gevers M, Van Der Mooren K, Stergiopulos N, Van Genderingen HR, Lafeber HN, Hack WW, et al. Bisferiens Peaks in the Radial Artery Pressure Wave during Patent Ductus Arteriosus in Newborn Infants: Relationship with Ascending Aortic Flow. Pediatr Res. 1996;40(1):163-8.

12. Lundell BPW. Pulse wave patterns in patent ductus arteriosus. Arch Dis Child. 1983;58:682-5.

13. Milstein JM, Riemenschneider TA, Goetzman BW, George L, Wennberg RP. Assessment of patent ductus arteriosus shunting using diastolic pressure analysis. J Pediatr. 1979;94(1):122-6.

14. Evans N, Moorcraft J. Effect of patency of the ductus arteriosus on blood pressure in very preterm infants. Arch Dis Child. 1992;67(10):1169-73. 
15. Han UJ, Cho HJ, Cho YK, Choi YY, Ma JS. Change in blood pressure and pulse pressure in preterm infants after treatment of patent ductus arteriosus with indomethacin. Korean Circ J. 2011;41(4):203-8.

16. Patterson SW, Starling EH. On the mechanical factors which determine the output of the ventricles. J Physiol. 1914;48(5):357-79.

17. de Waal K, Phad N, Collins N, Boyle A. Cardiac remodeling in preterm infants with prolonged exposure to a patent ductus arteriosus. Congenit Heart Dis. 2017;12(3):36472.

18. Takahashi Y, Harada K, Ishida A, Tamura M, Takada G. Left ventricular preload reserve in preterm infants with patent ductus arteriosus. Arch Dis Child - Fetal Neonatal Ed. 1994;71(2):F118-21.

19. Gevers M, Van Genderingen HR, Der Mooren K Van, Lafeber HN, Hack WWM, Westerhof N. Bisferiens Peaks in the Radial Artery Pressure Wave in Newborn Infants: A Sign of Patent Ductus Arteriosus. Pediatr Res. 1995;37(6):800-5.

20. Evans N. Preterm patent ductus arteriosus: A continuing conundrum for the neonatologist? Semin Fetal Neonatal Med. 2015;20(4):272-7.

21. Bose CL, Laughon M. Treatment to prevent patency of the ductus arteriosus: Beneficial or harmful? J Pediatr. 2006;148(6):713-4.

22. Benitz WE. Patent Ductus Arteriosus in Preterm Infants. Pediatrics. 2016;137(1):e20153730.

23. Chock V, Goel V, Palma J, Luh T, Wang N, Gaskari S, et al. Changing Management of the Patent Ductus Arteriosus: Effect on Neonatal Outcomes and Resource Utilization. Am J Perinatol. 2017;34(10):0990-5.

24. Benitz WE. Patent ductus arteriosus: to treat or not to treat? Arch Dis Child Fetal Neonatal Ed. 2012;97(2):F80-2.

25. Nemerofsky S, Parravicini E, Bateman D, Kleinman C, Polin R, Lorenz J. The Ductus Arteriosus Rarely Requires Treatment in Infants > 1000 Grams. Am J Perinatol. 2008;25(10):661-6.

26. Romagnoli V, Pedini A, Santoni M, Scutti G, Colaneri M, Pozzi M, et al. Patent ductus arteriosus in preterm infants born before 30 weeks' gestation: high rate of spontaneous closure after hospital discharge. Cardiol Young. 2018;28(08):995-1000.

27. Gevers M, Hack WW, Ree EF, Lafeber HN, Westerhof N. Arterial blood pressure wave forms in radial and posterior tibial arteries in critically ill newborn infants. J Dev Physiol. 1993;19(4):179-85.

28. Sun JX, Reisner AT, Mark RG. A signal abnormality index for arterial blood pressure waveforms. Comput Cardiol. 2006;33:13-6. 


\subsection{Supplementary materials}

\subsubsection{Artifact removal}

Artifacts were detected on a beat-to-beat basis per segment of 1000 beats. Two algorithms were used to detect artifacts, i.e. an altered signal abnormality index (SAI) [28] and a nearest neighbor waveform analysis. The altered version of the SAI focusses on large beat-to-beat changes that indicate severe artifacts. The nearest neighbor waveform analysis was used compare similarity between waveforms and can detect more subtle artifacts. If a beat was marked as an artifact, the beat previous to and following the artifact were also selected as an artifact.

\section{Altered signal abnormality index}

The SAI algorithm detects abnormal ABP waveforms using nine different features which indicate the waveform as an artifact if one of them exceeds a defined threshold. Five features are based on general waveform characteristics, namely the systolic, diastolic, mean and pulse arterial pressure and the heart rate. Three features calculate the difference of the current beat compared with the previous beat for systolic pressure, diastolic pressure and RR interval. The last feature calculates the mean of all downward slopes in the ABP waveform, which increases with high-frequency noise. In this study, this mean is divided by the pulse pressure, as the amplitude of the beat affects the downward slope.

Features were extracted per beat. The systolic and diastolic values were determined as described in the Methods section. The mean arterial pressure was the mean over one beat (diastolic to diastolic peak), while the pulse pressure was the difference between the diastolic and the subsequent systolic arterial pressure peak. If one of the features exceeded a set corresponding threshold, the beat was marked as an artifact. In case the threshold for a feature regarding the difference between the previous and the current beat was exceeded, the current beat was selected as an artifact. Thresholds were based on expert opinion. Thresholds were set to a systolic BP larger than $90 \mathrm{mmHg}$, a diastolic BP smaller than $10 \mathrm{mmHg}$, a mean $\mathrm{BP}$ smaller than $20 \mathrm{mmHg}$ or larger than $70 \mathrm{mmHg}$, a pulse pressure smaller than $2 \mathrm{mmHg}$ and a beat-to-beat heart rate smaller than $90 \mathrm{bpm}$ or larger than $220 \mathrm{bpm}$. The thresholds for the differences in systolic BP, diastolic BP and RR interval were respectively $15 \mathrm{mmHg}, 10 \mathrm{mmHg}$ and 0.5 seconds. The threshold for the summation of downward slopes was empirically determined at $-0.06 \mathrm{mmHg} / 8 \mathrm{~ms}$.

\section{Nearest neighbor waveform analysis}

Correctly measured waveforms have multiple similar beats in a segment of 1000 beats, corresponding to a small Euclidian distance to the most similar beat. Artifacts however do not or seldom have a similar waveform, resulting in a large Euclidian distance to the most similar beat. This study used a dynamic time warping based distance as an alternative to the Euclidian distance. Dynamic time warping is able to compare two waveforms correcting for non-linear differences in time such as a slowing of the waveform during the diastole. The corrected difference can accurately compare waveform shapes, independent of accelerations or decelerations. An artifact was defined as a beat that did not have five beats with a distance smaller than a certain threshold, in this case empirically set as 0.7 




\section{Chapter 4}

Improving prediction of favourable outcome after six months in patients with severe traumatic brain injury using physiological cerebral parameters in a multivariable logistic regression model

Frank C. Bennis, Bibi Teeuwen, Frederick A. Zeiler, Jan Willem Elting, Joukje van der Naalt, Pietro Bonizzi, Tammo Delhaas, Marcel J. Aries

Neurocrit Care. 2020;10.1007/s12028-020-00930-6 


\section{Abstract}

\section{Background}

Current severe traumatic brain injury (TBI) outcome prediction models calculate the chance of unfavourable outcome after six months based on parameters measured at admission. We aimed to improve current models with the addition of continuously measured neuromonitoring data within the first $24 \mathrm{~h}$ after intensive care unit neuromonitoring.

\section{Methods}

Forty-five severe TBI patients with intracranial pressure/cerebral perfusion pressure monitoring from two teaching hospitals covering the period May 2012 to January 2019 were analysed. Fourteen high-frequency physiological parameters were selected over multiple time periods after the start of neuromonitoring (o-6 h, o-12 h, o-18 h, o-24 h). Besides systemic physiological parameters and extended Corticosteroid Randomisation after Significant Head Injury (CRASH) score, we added estimates of (dynamic) cerebral volume, cerebral compliance and cerebrovascular pressure reactivity indices to the model. A logistic regression model was trained for each time period on selected parameters to predict outcome after six months. The parameters were selected using forward feature selection. Each model was validated by leave-one-out cross-validation.

\section{Results}

A logistic regression model using CRASH as the sole parameter resulted in an area under the curve (AUC) of 0.76 . For each time period, an increased AUC was found using up to five additional parameters. The highest AUC (0.90) was found for the $0-6 \mathrm{~h}$ period using 5 fiveparameters that describe mean arterial blood pressure and physiological cerebral indices.

\section{Conclusion}

Current TBI outcome prediction models can be improved by the addition of neuromonitoring bedside parameters measured continuously within the first $24 \mathrm{~h}$ after the start of neuromonitoring. As these factors might be modifiable by treatment during the admission, testing in a larger (multicenter) data set is warranted. 


\subsection{Introduction}

Severe traumatic brain injury (TBI) is defined as severe trauma to the brain and skull due to an external force. In Europe, 57.000 TBI-related deaths are reported each year [1]. TBI is the leading cause of death and severe disability in young adults [2]. The external force to the brain may result in ischaemia, contusions and haematomas. These processes lead to swelling, rise in intracranial pressure (ICP), decrease in cerebral perfusion pressure (CPP) and finally cerebral ischaemia [2]. Intensive care unit (ICU) admission with organ support is necessary in comatose TBI patients to overcome secondary damage. Severe or moderate disability is common in surviving patients, which makes TBI a large burden for patients, families and society [3]. An accurate prediction of outcome would be helpful, as it would support the clinical team in decision-making and discussions with the family during ICU admission.

Models such as the (extended) model based on data from the Corticosteroid Randomisation after Significant Head Injury (CRASH) study are developed to predict the six-month individual outcome [4,5]. These models primarily use baseline demographics and factors related to the primary injury to predict outcome. However, confounding factors, consequences of the initial trauma (like brain swelling, metabolic crises and inflammation) and the individual response to therapy during ICU admission are not included. Because full supportive care for a certain amount of time from initial presentation is recommended to maximize the potential for recovery from primary and secondary damage [6], extending prognostic models with early physiological monitoring data might improve the outcome prediction accuracy as has been shown in studies on the ICU for pathologies other than TBI [7-9].

Commonly used parameters for continuous hemodynamic monitoring in the ICU are heart rate (HR) and mean arterial blood pressure (MAP). For cerebral monitoring, the guidelines recommend ICP and CPP monitoring [10,11]. These parameters depend heavily on the treatment given and are associated with mortality $[12,13]$ but are limited in their correlation with unfavourable outcome. Therefore, the use of additional cerebral parameters such as the cerebral compliance and autoregulation has been suggested for therapy guidance [14]. Dynamic cerebral autoregulation parameters such as the cerebrovascular pressure reactivity index (PRx) are gaining more interest because these parameters are correlated independently with TBI outcome [14-17].

In this retrospective study, we aim to develop a model that combines the prediction of the CRASH model with continuously measured general and brain-specific monitoring parameters in severe TBI patients on day one after the start of neuromonitoring. We hypothesize that extending the prediction model improves outcome prediction and may assist decisionmaking during the ICU stay. 


\subsection{Methods}

\subsubsection{Design and subjects}

Patients from the ICU of the University Medical Centre Groningen (UMCG) and Maastricht University Medical Centre (MUMC), both in the Netherlands, were retrospectively analysed. Data were included from two centres to increase the number of patients. In both the centres, medical ethical committees approved anonymized physiological, diagnostic, clinical and outcome data collection. The need for informed consent was waived in Groningen. In Maastricht, informed consent was obtained from the closest relative. Inclusion occurred between May 2012 and March 2015 in the UMCG and between April 2017 and January 2019 in the MUMC. Inclusion criteria were (1) severe TBI and (2) ICP/CPP monitoring. Exclusion criteria were (1) moribund at admission, (2) pregnancy, (3) monitoring started $>24 \mathrm{~h}$ after trauma, (4) loss to follow-up or (5) incomplete baseline data for the extended CRASH score.

\subsubsection{Data collection}

Outcome after six months was obtained by consultation over the phone by a clinician. The outcome of patients was scored on the five-point Glasgow Outcome Scale (GOS). The GOS was subsequently dichotomized to unfavourable (dead, vegetative state or severe disability (GOS 1-3)) or favourable (mild disability or full recovery (GOS 4-5)) outcome. Mortality after 14 days was not evaluated as this was not standardly collected. The patients were treated by the same neuro-intensivist in both hospitals (MJ Aries).

Electrocardiogram, arterial blood pressure (ABP) and ICP were recorded at $250 \mathrm{~Hz}$ using $\mathrm{ICM}+$ software (www.icmplus.neurosurg.cam.ac.uk) running on a bedside computer. In Groningen, an external ventricular drain was used with an electronic (ICP) sensor in the tip for intraventricular pressure monitoring and optional cerebral spinal fluid drainage capacity (Neurovent, RAUMEDIC AG, Helmbrechts, Germany). In Maastricht, a parenchymal sensor was used. ABP was zeroed at heart level in Groningen. In Maastricht, ABP was zeroed at heart level up to 2018, after which $\mathrm{ABP}$ was measured at the brain level. Corresponding HR, ABP and ICP were down-sampled to one sample/min. PRx was calculated as the moving Pearson correlation of 30 consecutive 10-s non-overlapping moving window averages of ABP and ICP, updated every minute, resulting in one averaged correlation value per minute. The PRx index indicates the intactness of the cerebrovascular reactivity. With an intact cerebrovascular reactivity, a slow increase in $\mathrm{ABP}$ will be counteracted by cerebral vasoconstriction, leading to a decrease in cerebral volume and subsequent in ICP. If the cerebrovascular reactivity is impaired, the cerebral volume and ICP will rise as a result of an increase in ABP. Therefore, the correlation coefficient will be negative or around zero in the case of an intact cerebrovascular reactivity, and positive when the cerebrovascular reactivity is impaired. Similar correlationbased parameters calculate the correlation coefficient in the same way, but by using different input and output parameters. These correlation-based parameters are the correlation coefficient between: ABP and pulse amplitude of ICP (AMP) (pressure amplitude index (PAx)), which describes the cerebrovascular reactivity; moving correlation coefficient between AMP and CPP (RAC), which includes information about the cerebral compensatory 
reserve and cerebrovascular reactivity; moving correlation coefficient between AMP and ICP (RAP), which describes the intracranial compliance. For more in-depth information about these dynamic parameters, we refer to literature $[16,18]$. The extended CRASH score was calculated with basic clinical parameters obtained at admission [4].

\subsubsection{Physiological parameters and methodology}

MATLAB (2018A, The MathWorks, Natick, MA, USA) was used for all analyses. Outliers were replaced in HR, MAP and ICP with the filloutliers function. The algorithm replaced data points more than six median absolute deviations above or below the median by a linearly interpolated value using the previous and the next data point. No outliers were removed in parameters describing the trends in cerebral compliance, compensatory reserve and autoregulation, as individual data points are known to be noisy [19].

We investigated which of four data periods after the start of neuromonitoring were most informative to predict six-month outcome. These periods contained data from $\mathrm{o}-6 \mathrm{~h}, \mathrm{o}-12 \mathrm{~h}$, $\mathrm{o}-18 \mathrm{~h}$ and $\mathrm{o}-24 \mathrm{~h}$ after the start of monitoring. Missing data were not replaced. If a period contained less data than $50 \%$ of the total length or less than three hours additional to the previous period, the period was excluded. We chose to exclude only time periods and not the entire patient, in order to represent the clinical setting. In total, 15 parameters for each period were selected representing different physiological domains: (1) average ICP, ABP and HR; (2) average PRx, PAx, RAP and RAC; (3) the slope of a linear line fitted on the PRx, PAx and RAC (as an indicator how these parameters progress over time); (4) the amount of impairment of autoregulatory parameters, defined as the area of the signal above a set threshold divided by the total amount of samples: for the PRx (threshold > 0.35), PAx (> 0.25), RAC (>- 0.05) and ICP (> $2 \mathrm{mmHg}$ ) [12]; and (5) individual unfavourable outcome risk score (CRASH, \%). This resulted in 15 values per time segment.

\subsubsection{Logistic regression modelling}

A logistic regression model was trained based on the above parameters, called the 'combined' model. To train the model with those parameters that are most predictive of outcome, the algorithm will first rank the parameters on their respective predictive power (Figure 4.1).

\section{Ranking of parameters}

To rank the parameters, leave-one-out cross-validation (LOOCV) [20] was combined with forward feature selection (FFS) (Figure 4.1A) [21]. LOOCV splits the data set 45 times (called folds) in a training set containing all but one and a test set containing the remaining patient. For each fold, the training set was used to determine the optimal order of parameters by FFS, from best to worst. The CRASH risk score was fixed to be the first ranked parameter. This results in an optimal order of parameters for each patient when not including that patient in the FFS. To define the overall optimal order of parameters, the $\mathrm{k}^{\text {th }}$ optimal parameter is selected as the most occurring parameter at position $\mathrm{k}$ from all $45 \mathrm{LOOCV}$ models. This is continued until all parameters are included, resulting in an overall ranking of the 15 parameters. 


\section{Selection of variables}

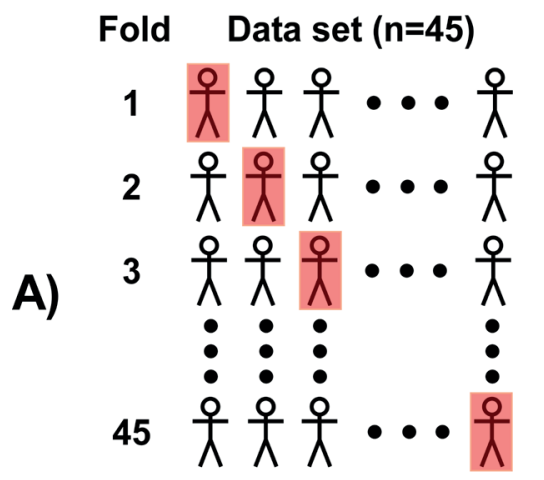

Feature order

$11513 \bullet \bullet 6$

$11013 \bullet \bullet 7$

$11513 \bullet \bullet 6$

$:::$ :

$1154 \bullet \bullet 6$

$11513 \bullet \bullet 6$

\section{Model training}

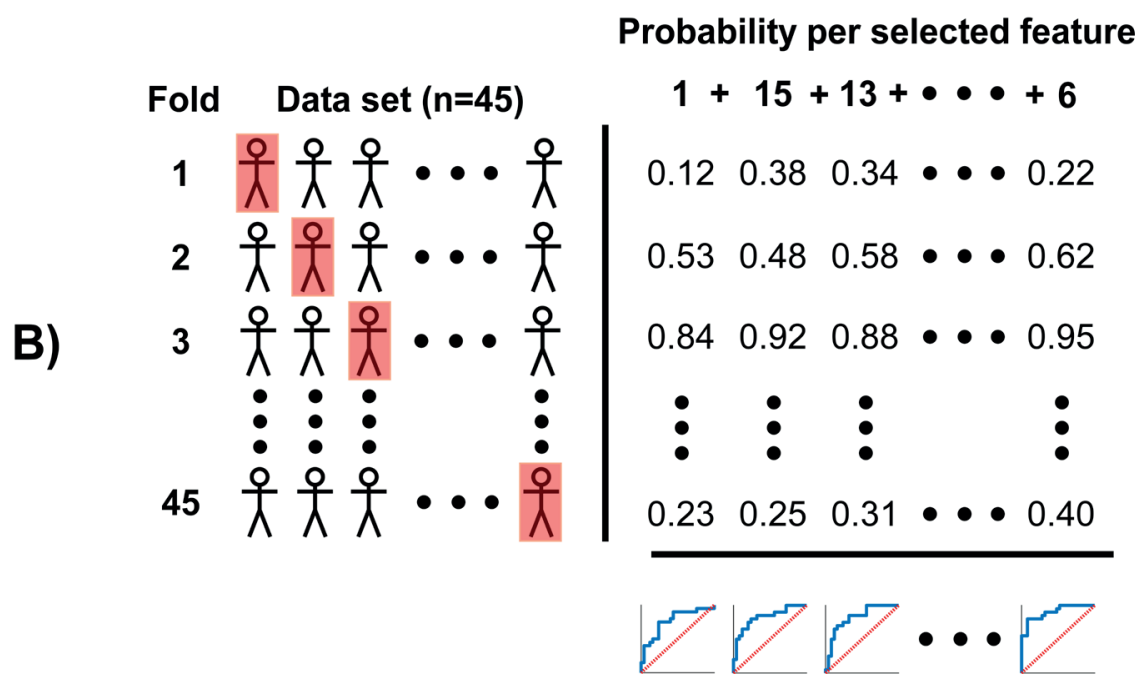

Figure 4.1: A. Selection of the ideal order of parameters for the selected time period (for instance o-6 h) using leave-one-out cross-validation and forward feature selection. The data set selects a different test set (red square) for each fold. Parameters are ordered subsequently for each fold. The parameters which are seen most often for each column are selected as the order for the final model. B. Model training using the previous selected parameters, each time adding the next best parameter. The data again selects a different test set for each fold. A logistic regression model is trained and tested for each fold, resulting in the probability of an unfavourable outcome for each subject. An ROC curve is created using this probability for all subjects over a single number of features included. The ROC with the best AUC is selected as the final model for this time segment. 


\section{Training the model}

To train the model, the data set is again divided by LOOCV (Figure 4.1B) and training and testing are performed for each fold. Starting with the neuromonitoring-derived parameter that was ranked highest as single input, a logistic regression model is trained on the training set and tested on the test set for each split. The test set output is the probability that the patient has an unfavourable outcome. As this is repeated for each fold, the probability of an unfavourable outcome is predicted for each patient. Thereafter, a model that included the two highest ranked parameters was trained and tested, again resulting in an individual probability of unfavourable outcome. This is repeated until all parameters are added. The number of parameters with the highest area under the curve (AUC) in the corresponding ROC curve is selected as the best model for that time period. A common problem in machine learning is overfitting, which occurs when the number of parameters is too large compared to sample size. Since the number of patients is limited, a maximum of six parameters were included. The selection of parameters and model training was performed for each time period.

The accuracy of the model, expressed in correctly predicted patients, is based on the optimal cut-off value determined by the Youden's index [22]. The performance of the CRASH model and the different combined models will be compared using ROC curves, the AUC values and the prediction accuracy. We decided not to statistically compare AUC curves due to the limited sample size, and hence, results should be interpreted at a qualitative level. Model calibration is assessed by visualization of the predicted probabilities versus the actual outcome.

\subsection{Results}

Sixty-two patients were eligible for inclusion. Two patients did not have any data. Eleven patients were excluded because monitoring started $>24 \mathrm{~h}$ after the trauma. The outcome was unknown in four of the remaining 49 patients. In total, 45 patients were available for analysis.

The patients had a median age of 41 years (interquartile range (IQR) 24-57) and a median admission Glasgow Coma Scale (GCS) of 7 (IQR 5-9). Thirty-three patients were male (73.3\%). In 11 patients, ABP was zeroed at the brain level. In three patients, a secondary decompressive craniectomy was performed. In 29 out of 45 patients, the mechanism of injury involved road traffic accidents. In 13 patients, the mechanism of injury was a fall of height. The remaining three patients involved an assault, metal against head and a hit by a tree. The patients had a median Marshall computed tomography score of 2, with the 25 and 75 percentile also at 2. Four patients had evacuated mass lesions. Dividing patients according to the GOS, 13 (28.9\%) died, 1 (2.2\%) was in a vegetative state, 6 (13.3\%) experienced severe disabilities, 10 (22.2\%) had moderate disabilities and 15 (33.3\%) made a good recovery. Twenty patients (44.4\%) had an unfavourable outcome. All 45 patients were included in the $\mathrm{o}-6 \mathrm{~h}$ and $\mathrm{o}-12 \mathrm{~h}$ group. We excluded one patient (GOS score 5 ) in the $\mathrm{o}-18 \mathrm{~h}$ group, whereas we had to exclude four patients (GOS score 4-5) in the $0-24 \mathrm{~h}$ group because data collection was stopped due to early removal of the ICP monitor as a result of low ICP values. The mean parameter values are given in Supplementary Table S4.1. 


\subsubsection{Performance of different models}

The CRASH model performs adequate for all periods (AUC 0.75-0.76, accuracy 75.0-75.6\%) (Table 4.1, Figure 4.2). Slight deviations in CRASH performance are due to the fact that not all time periods had an equal number of subjects. The combined model shows a high AUC and accuracy for each time period, especially in the early monitoring period (o-6 h, AUC 0.90, accuracy 86.7\%). The parameters included per time segment are shown in Table 4.2. The logistic regression model coefficients and thresholds for favourable vs unfavourable prediction are given in Supplementary Table S4.2. The calibration curves indicate a systematic underestimation for the lower predicted probabilities, whilst a systematic overestimation for the higher predicted probabilities is observed (Figure 4.3). However, as the data set used in this study is fairly small and the magnitude of error is not severe, calibration is deemed acceptable [23].

A)
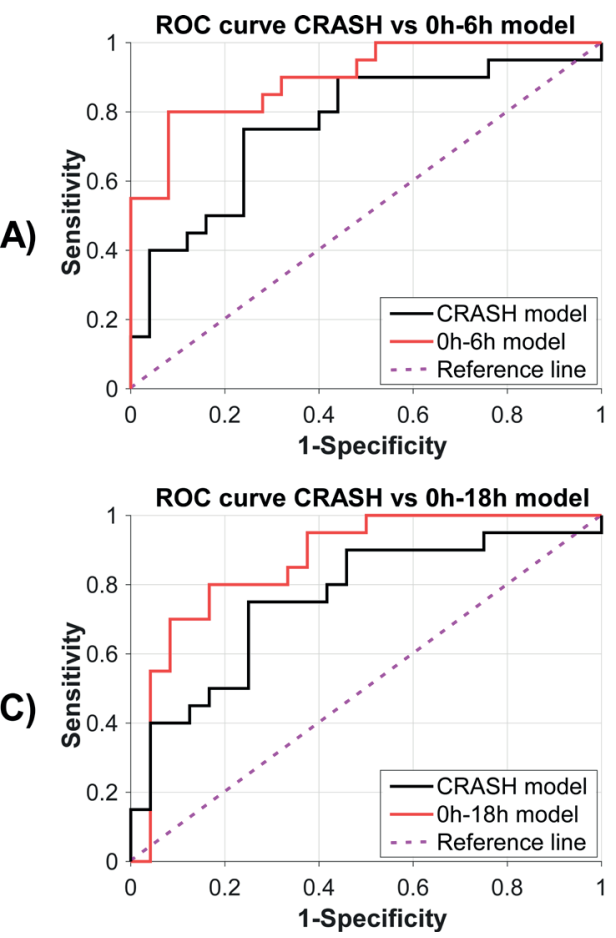

B)
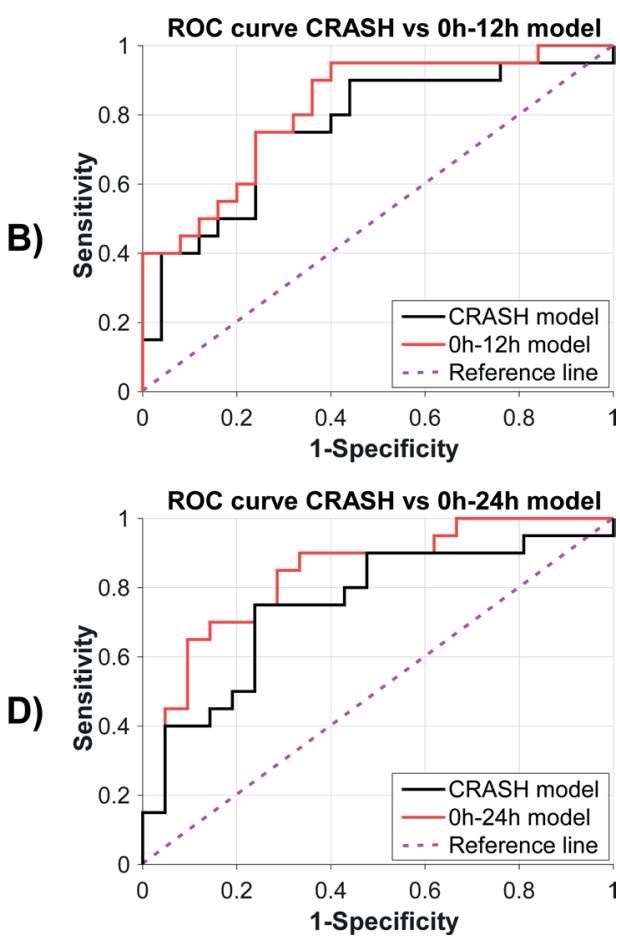

Figure 4.2: Models predicting unfavourable outcome in severe TBI patients and ICU admission. ROC curves of the CRASH model and the combined model for each time period. High AUC values are seen for the logistic model predicting unfavourable outcome, especially for the early monitoring period (Figure $4.2 \mathrm{~A})$ 


\begin{tabular}{|lllll|}
\hline \multirow{2}{*}{ Time period } & \multicolumn{2}{l}{ CRASH risk score } & \multicolumn{2}{l|}{ Combined models } \\
& AUC (CI) & $\begin{array}{l}\text { Prediction } \\
\text { accuracy (\%) }\end{array}$ & AUC (CI) & $\begin{array}{l}\text { Prediction } \\
\text { accuracy (\%) }\end{array}$ \\
\hline $\mathrm{o}-6 \mathrm{~h}(\mathrm{n}=45)$ & $0.76(0.62-0.91)$ & 75.6 & $0.9(0.8-1)$ & 86.7 \\
\hline $\mathrm{o}-12 \mathrm{~h}(\mathrm{n}=45)$ & $0.76(0.62-0.91)$ & 75.6 & $0.82(0.69-0.95)$ & 75.6 \\
\hline $\mathrm{o}-18 \mathrm{~h}(\mathrm{n}=44)$ & $0.76(0.61-0.9)$ & 75.0 & $0.87(0.76-0.98)$ & 81.8 \\
\hline $0-24 \mathrm{~h}(\mathrm{n}=41)$ & $0.75(0.6-0.9)$ & 75.6 & $0.84(0.71-0.96)$ & 78.0 \\
\hline
\end{tabular}

Table 4.1: The AUC and prediction accuracy for the best model using the CRASH and the combined model for each time period. Higher AUC values and prediction accuracies can be seen for the combined model for predicting unfavourable outcome after severe TBI and ICU admission
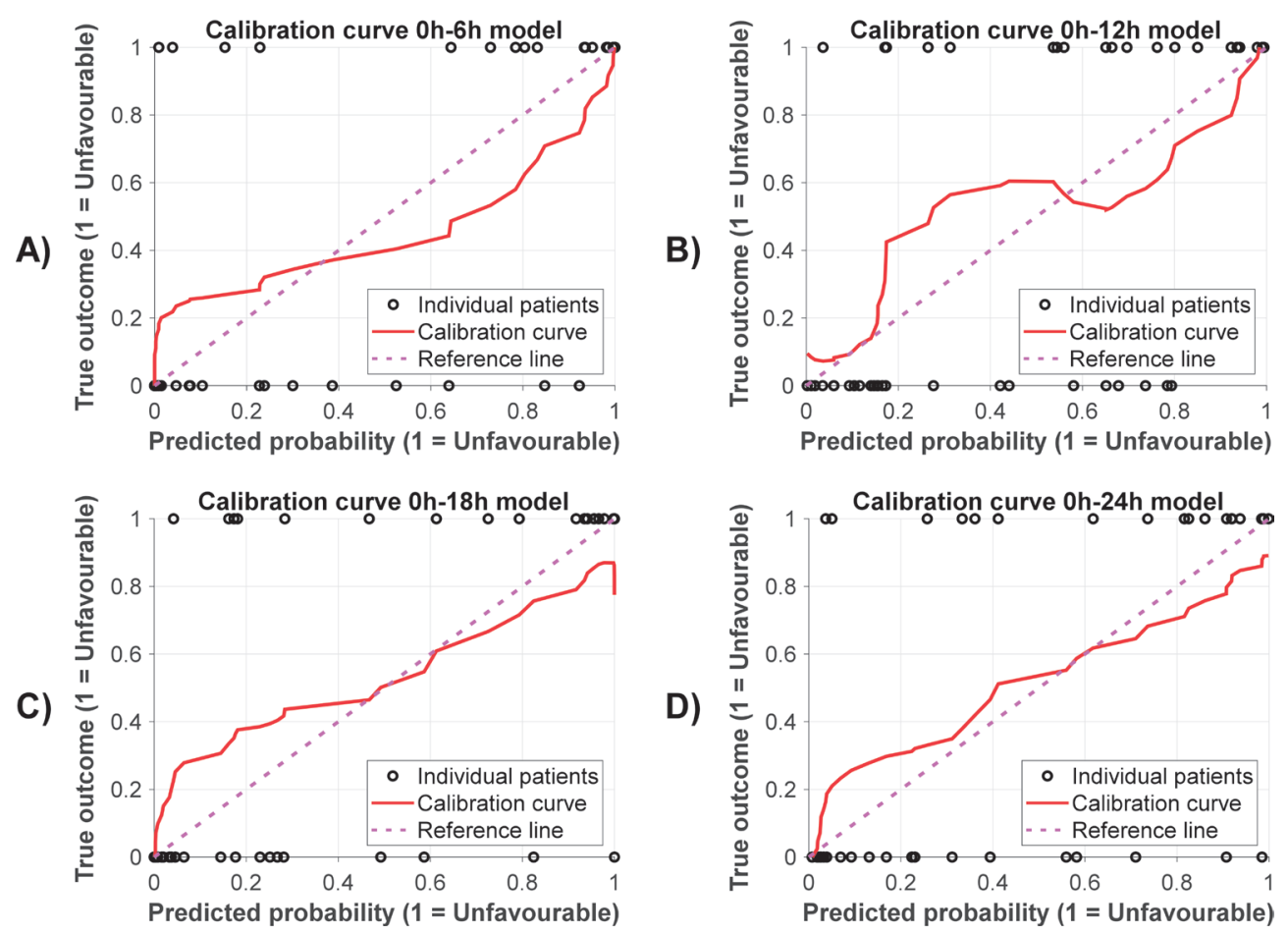

Figure 4.3: Calibration plots of the combined models predicting unfavourable outcome in severe traumatic brain injury patients. A systematic underestimation is seen for the lower predicted probabilities, whilst a systematic overestimation is seen for higher predicted probabilities. 


\begin{tabular}{lclllll}
\multicolumn{1}{c}{$\begin{array}{l}\text { Time } \\
\text { segment }\end{array}$} & \multicolumn{2}{c}{ Parameter included } & & & & \\
& First & Second & Third & Fourth & Fifth & Sixth \\
\hline CRASH & CRASH & & & & & \\
oh-6h & CRASH & Mean ABP & Slope PAx & Slope PRx & Slope RAC & \\
oh-12h & CRASH & $\begin{array}{l}\text { Impairment } \\
\text { PRx }\end{array}$ & Mean ABP & & & \\
oh-18h & CRASH & Mean PRx & Mean PAx & Mean ABP & Impairment & Slope RAC \\
& CRASH & $\begin{array}{l}\text { Impairment } \\
\text { PRx }\end{array}$ & Slope PAx & Slope PRx & Mean ABP & \\
\hline oh-24h & CRAC & & & & \\
& & & & & & \\
\hline
\end{tabular}

Table 4.2: Parameters included per time segment. Ordering is the sequence in which forward feature selection picked the parameters.

\begin{tabular}{lcccccc}
$\begin{array}{l}\text { Time } \\
\text { segment }\end{array}$ & \multicolumn{2}{c}{ True GOS score } & & & & \\
& $\mathbf{1}$ & $\mathbf{2}$ & $\mathbf{3}$ & $\mathbf{4}$ & $\mathbf{5}$ & Total \\
\hline CRASH & $2 / 13$ & $1 / 1$ & $2 / 6$ & $3 / 10$ & $3 / 15$ & $11 / 45$ \\
\hline oh-6h & $1 / 13$ & $0 / 1$ & $3 / 6$ & $0 / 10$ & $2 / 15$ & $6 / 45$ \\
\hline oh-12h & $3 / 13$ & $0 / 1$ & $2 / 6$ & $2 / 10$ & $4 / 15$ & $11 / 45$ \\
\hline oh-18h & $2 / 13$ & $0 / 1$ & $2 / 6$ & $2 / 10$ & $2 / 14$ & $8 / 44$ \\
\hline oh-24h & $6 / 13$ & $0 / 1$ & $1 / 6$ & $2 / 8$ & $0 / 13$ & $9 / 41$ \\
\hline
\end{tabular}

Table 4.3: Number of false favourable (true GOS score of $1,2,3$ ) and false unfavourable predictions (true GOS score of 4,5 ) vs total number of subjects per true GOS score

\subsubsection{False classification}

Both the CRASH and the combined models classified a few more patients to have a favourable than an unfavourable outcome (Table 4.3). The number of misclassified subjects was comparable over all GOS outcomes. The percentage of patients who were classified to have a favourable outcome but actually died ranged from $7.7 \%$ ( 1 out of 13 ) to $46.2 \%$ (6 out of 13 ). The percentage of patients who were predicted to have an unfavourable outcome but actually had a GOS score of 5 ranged from o to $26.7 \%$ (4 out of 15). The combined model using data of $0-6 \mathrm{~h}$ showed the highest AUC, the least mortality misclassifications and the second-best good recovery misclassifications. 


\subsection{Discussion}

This pilot study aimed to develop a model that combined baseline parameters with continuously measured general and brain-specific monitoring parameters in admitted severe TBI patients to improve the prediction accuracy for the six-month clinical outcome. The combined models showed high AUC values when using data from $\mathrm{o}-6 \mathrm{~h}$ up to $0-24 \mathrm{~h}$ period, with the highest performance when using data of the first $6 \mathrm{~h}$ (Figure 4.2, Table 4.1). Furthermore, an increase in prediction accuracy was found in three out of four time periods, with the best performance when neuromonitoring data of the first $6 \mathrm{~h}$ were used. Our results are in line with our hypothesis that current TBI outcome prediction models can be improved by the addition of early neuromonitoring data.

Of special interest are the parameters selected by the model. Mean ABP was included for each time period, with lower ABP resulting in a higher chance of unfavourable outcome. This is in line with current clinical practice, as ABP is part of all treatment protocols for critically ill patients. Lower systolic blood pressures before and during ICU admission are related to higher mortality rates in TBI patients $[13,24]$. The finding that $A B P$ was selected in every model-despite the fact that different ABP zeroing levels were applied in patients-is interesting because autoregulation-based parameters are more likely to be included by the model due to the fact that they do not rely on the absolute level of ABP. Additional analyses of models including CRASH and mean ABP as sole parameters show an AUC and prediction accuracy of 0.82 and $77.8 \%, 0.79$ and $75.6 \%, 0.76$ and $72.7 \%$ and 0.74 and $75.6 \%$ for the $\mathrm{o}-6 \mathrm{~h}, \mathrm{o}-12 \mathrm{~h}, \mathrm{o}-18 \mathrm{~h}$ and $\mathrm{o}-24 \mathrm{~h}$ time spans, respectively. These findings indicate that particular combinations of physiological derangements may be (prognostically) important (for example the combination of low ABP in a situation with impaired autoregulation). Subsequent parameters were all correlation-based parameters describing trends in cerebrovascular (autoregulation) reactivity or cerebral compensatory reserve. Of these correlation-based parameters, different characteristics were selected (slope, mean and amount of impairment). These findings indicate that not only a general ICU parameter (ABP) but also specific parameters representing the unique (global) cerebral homoeostasis and protection mechanisms contain prognostic information. We speculate that trends in these parameters can be used in addition to baseline prognostic parameters to aid decision-making and discussion between the clinical team and families during the ICU admission period. 
The finding that the effect of including neuromonitoring data in a prediction model for sixmonth outcome is the largest when using data from the 'early' $\mathrm{o}-6 \mathrm{~h}$ time period may be because we studied trauma patients in which treatment was focused on controlling ICP as the main goal. These treatment effects may have reduced the effect of the selected parameters in the combined model. However, in the early phase of admission, the patient may not yet be fully stabilized and thus the parameters may reflect the deranged physiological status of the patient better. Including treatment intensity level may account for treatment given in a later stage. The treatment effect may be most apparent in the fact that ICP was never selected, as current therapy protocols are primarily focused on controlling ICP. Recent literature has shown that cerebrovascular reactivity/autoregulation parameters, however, appear to remain relatively independent of the ICP-guided treatment [25-27]. As cerebrovascular reactivity or autoregulation status might be modifiable by directed (perfusion) therapies started as early as possible, our findings might add retrospective evidence for the call for prospective testing.

\subsubsection{Limitations}

This study has several limitations. First, the sample size of this study is small. Therefore, we did not have the possibility to use a separate test set. Instead, we performed feature selection in a separate cross-validation before training the model. Selection of parameters by FFS may vary between subjects in the case of correlation between parameters, as the information between parameters is quite similar. As some parameters are correlated in this study, the order of parameters in different patients as selected by FFS varied. Therefore, variability in feature selection and subsequent low performance would be seen if FFS was performed in the cross-validation splits together with prediction. Although the current method might introduce a slight bias, it ensures that each model uses the same features. To improve training and ensure correct generalization, we recommend increasing the sample size and validate the found models on an external data set. Second, the parameters used show correlation, especially parameters describing autoregulation such as PRx and PAx. The feature selection algorithm used in this method does not take correlation into account. Although this is partly solved due to the selection of parameters that perform best most often, it is recommended to use algorithms that are capable of handling correlation in parameters, such as lasso logistic regression. To test the influence of correlation between parameters, model performance was evaluated once without PRx and once without PAx. AUC was the highest using both PRx and PAx in three out of the four time segments and equal in the remaining one time segment (data not shown). Therefore, although the correlation is present, including both parameters improves prediction accuracy. We hypothesize that both might contain different cerebral hemodynamic information. Third, selection of the 14 monitoring-based parameters was based on availability and proven relationship to outcome in the literature. The parameters in this study are mainly perfusion related, whilst brain oxygenation or metabolism is not considered. Adding the latter to the model, for instance using near-infrared spectroscopy or parenchymal brain tissue oxygenation, may further increase the predictive value. Fourth, it is possible that events occur after the first $24 \mathrm{~h}$, such as a deterioration or improvement in the measured physiological parameters, complications or independent issues, such as unrelated death after discharge. Future models should consider incorporating such long-term deviations in addition to the early-phase parameters. Fifth, the combined model included ICU-admitted 
severe TBI patients, whilst the CRASH model was trained on all TBI patients with a GCS lower than 14 [4]. Therefore, the CRASH model is not optimized for our specific data set. If the initial CRASH model accuracy would be higher, the addition of early neuromonitoring data may result in even higher prediction accuracies than currently found. In future studies, we will include the model created on the International Mission for Prognosis and Analysis of Clinical Trials (IMPACT score) in TBI database [5], which may result in additional info and thus better outcome prediction. Sixth, the model did not directly take the influence of treatment into account. In future work, the (intracranial hypertension) treatment intensity level is worth adding as a separate parameter to the model. Last, this study used the time from the start of neuromonitoring to divide data in time segments. However, the start of neuromonitoring (data collection) may be postponed due to for example operator availability or delayed ICU arrival due to urgent (life-saving) surgery. Dividing data according to time after trauma and adding parameters describing time and procedures from trauma to ICU admission/data collection may improve outcome prediction.

Prognostication in ICU patients can be improved by physiological parameters. Meiring et al. [7] showed that common physiological parameters such as HR and MAP and treatment given can predict mortality on the ICU on subsequent days. Other applications are prediction of delayed cerebral ischaemia after subarachnoid haemorrhage [9], prediction of favourable neurological outcome among children on the ICU with critical illness [8] or prediction of impending sepsis in neonates [28]. Although it also has been attempted to use physiological parameters to predict outcome 6 to 12 months after TBI, data used are solely measured before admission or incorporate the whole ICU admission period, hampering (early) clinical assistance $[4,5,29-31]$.

\subsection{Conclusion}

This study showed that the inclusion of (complex) physiological data of the first $24 \mathrm{~h}$ after admission improves the prediction of the six-month outcome in TBI patients. The main perfusion-related parameters included are ABP and parameters describing cerebral compliance and autoregulation. As these parameters might be modifiable by treatment during the admission, testing in a larger (multicenter) data set is warranted. 


\subsection{References}

1. Majdan M, Plancikova D, Brazinova A, Rusnak M, Nieboer D, Feigin V, et al. Epidemiology of traumatic brain injuries in Europe: a cross-sectional analysis. Lancet Public Heal 2016;1(2):e76-83.

2. Maas AI, Stocchetti N, Bullock R. Moderate and severe traumatic brain injury in adults. Lancet Neurol 2008;7(8):728-41.

3. Stocchetti N, Zanier ER. Chronic impact of traumatic brain injury on outcome and quality of life: a narrative review. Crit Care 2016;20(1):148.

4. Perel P, Arango M, Clayton T, Edwards P, Komolafe E, Poccock S, et al. Predicting outcome after traumatic brain injury: practical prognostic models based on large cohort of international patients. BMJ 2008;336(7641):425-9.

5. Steyerberg EW, Mushkudiani N, Perel P, Butcher I, Lu J, McHugh GS, et al. Predicting Outcome after Traumatic Brain Injury: Development and International Validation of Prognostic Scores Based on Admission Characteristics. PLoS Med 2008;5(8):e165.

6. Pratt AK, Chang JJ, Sederstrom NO. A Fate Worse Than Death: Prognostication of Devastating Brain Injury. Crit Care Med 2019;47(4):591-8.

7. Meiring C, Dixit A, Harris S, MacCallum NS, Brealey DA, et al. Optimal intensive care outcome prediction over time using machine learning. PLoS One 2018;13(11):e0206862.

8. Gupta P, Rettiganti M, Gossett JM, Daufeldt J, Rice TB, Wetzel RC. Development and Validation of an Empiric Tool to Predict Favorable Neurologic Outcomes Among PICU Patients. Crit Care Med 2018;46(1):108-15.

9. Park S, Megjhani M, Frey HP, Grave E, Wiggins C, Terilli KL, et al. Predicting delayed cerebral ischemia after subarachnoid hemorrhage using physiological time series data. $\mathrm{J}$ Clin Monit Comput 2019;33(1):95-105.

10. Carney N, Totten AM, O’Reilly C, Ullman JS, Hawryluk GW, Bell MJ, et al. Guidelines for the Management of Severe Traumatic Brain Injury. Neurosurgery 2017;80(1):6-15.

11. Stocchetti N, Maas AIR. Traumatic Intracranial Hypertension. N Engl J Med 2014;370(22):2121-30.

12. Zeiler FA, Ercole A, Cabeleira M, Zoerle T, Stocchetti N, Menon DK, et al. Univariate comparison of performance of different cerebrovascular reactivity indices for outcome association in adult TBI: a CENTER-TBI study. Acta Neurochir 2019;161(6):1217-27.

13. Merck LH, Yeatts SD, Silbergleit R, Manley GT, Pauls Q, Palesch Y, et al. The Effect of Goal-Directed Therapy on Patient Morbidity and Mortality After Traumatic Brain Injury. Crit Care Med 2019;47(5):623-31.

14. Le Roux P, Menon DK, Citerio G, Vespa P, Bader MK, Brophy GM, et al. Consensus summary statement of the International Multidisciplinary Consensus Conference on Multimodality Monitoring in Neurocritical Care. Intensive Care Med 2014;40:1189-209.

15. Sorrentino E, Diedler J, Kasprowicz M, Budohoski KP, Haubrich C, Smielewski P, et al. Critical Thresholds for Cerebrovascular Reactivity After Traumatic Brain Injury. Neurocrit Care 2012;16(2):258-66.

16. Zeiler FA, Donnelly J, Smielewski P, Menon DK, Hutchinson PJ, Czosnyka M. Critical Thresholds of Intracranial Pressure-Derived Continuous Cerebrovascular Reactivity Indices for Outcome Prediction in Noncraniectomized Patients with Traumatic Brain Injury. J Neurotrauma 2018;35(10):1107-15. 
17. Donnelly J, Czosnyka M, Adams H, Robba C, Steiner LA, Cardim D, et al. Individualizing Thresholds of Cerebral Perfusion Pressure Using Estimated Limits of Autoregulation. Crit Care Med 2017;45(9):1464-71.

18. Zeiler FA, Donnelly J, Menon DK, Smielewski P, Hutchinson PJA, Czosnyka M. A Description of a New Continuous Physiological Index in Traumatic Brain Injury Using the Correlation between Pulse Amplitude of Intracranial Pressure and Cerebral Perfusion Pressure. J Neurotrauma 2018;35(7):963-74.

19. Czosnyka M, Smielewski P, Kirkpatrick P, Laing RJ, Menon D, Pickard JD. Continuous Assessment of the Cerebral Vasomotor Reactivity in Head Injury. Neurosurgery 1997;41(1):11-9.

20. Hastie T, Tibshirani R, Friedman J. Model Assessment and Selection. In: The Elements of Statistical Learning: Data Mining, Inference, and Prediction, 2d edn. 2009. p. 745.

21. Guyon I, Elisseeff A. An Introduction to Variable and Feature Selection. J Mach Learn Res 2003;3:1157-82.

22. Youden WJ. Index for rating diagnostic tests. Cancer 1950;3(1):32-5.

23. Austin PC, Steyerberg EW. Graphical assessment of internal and external calibration of logistic regression models by using loess smoothers. Stat Med. 2014;33:517-35

24. Spaite DW, Hu C, Bobrow BJ, Chikani V, Sherrill D, Barnhart B, et al. Mortality and Prehospital Blood Pressure in Patients With Major Traumatic Brain Injury. JAMA Surg 2017;152(4):360.

25. Zeiler FA, Ercole A, Beqiri E, et al. Cerebrovascular reactivity is not associated with therapeutic intensity in adult traumatic brain injury: a CENTER-TBI analysis. Acta Neurochir 2019;161(9):1955-64.

26. Donnelly J, Czosnyka M, Adams H, et al. Twenty-Five Years of Intracranial Pressure Monitoring After Severe Traumatic Brain Injury: A Retrospective, Single-Center Analysis. Neurosurgery 2019;85(1):E75-82.

27. Weersink CS, Aries MJ, Dias C, Liu MX, Kolias AG, Donnelly J, et al. Clinical and Physiological Events That Contribute to the Success Rate of Finding "Optimal” Cerebral Perfusion Pressure in Severe Brain Trauma Patients. Crit Care Med 2015;43(9):1952-63.

28. Moorman JR, Delos JB, Flower AA, Cao H, Kovatchev BP, Richman JS, et al. Cardiovascular oscillations at the bedside: early diagnosis of neonatal sepsis using heart rate characteristics monitoring. Physiol Meas 2011;32(11):1821-32.

29. Andrews PJ, Sleeman DH, Statham PF, McQuatt A, Corruble V, Jones PA, et al. Predicting recovery in patients suffering from traumatic brain injury by using admission variables and physiological data: a comparison between decision tree analysis and logistic regression. J Neurosurg 2002;97(2):326-36.

30. Walker WC, Stromberg KA, Marwitz JH, Sima AP, Agyemang AA, Graham KM, et al. Predicting Long-Term Global Outcome after Traumatic Brain Injury: Development of a Practical Prognostic Tool Using the Traumatic Brain Injury Model Systems National Database. J Neurotrauma 2018;35(14):1587-95.

31. Wartenberg KE, Hwang DY, Haeusler KG, Muehlschlegel S, Sakowitz OW, Madžar D, et al. Gap Analysis Regarding Prognostication in Neurocritical Care: A Joint Statement from the German Neurocritical Care Society and the Neurocritical Care Society. Neurocrit Care 2019;1-14. 


\subsection{Supplementary materials}

\section{Mean (SD)}

oh-6h oh-12h oh-18h oh-24h

$(n=45) \quad(n=45) \quad(n=44) \quad(n=41)$

CRASH risk $\quad 58.9(21.9) \quad 58.9(21.9) \quad 59.4(21.9) \quad 60.7(21.4)$ score (\%)

\begin{tabular}{|c|c|c|c|c|c|}
\hline \multirow{7}{*}{ Mean } & $\begin{array}{l}\text { ICP } \\
(\mathrm{mmHg})\end{array}$ & $11.1(5.4)$ & $11.6(5.4)$ & $12.2(5 \cdot 7)$ & $12.4(5.8)$ \\
\hline & $\begin{array}{l}\mathrm{ABP} \\
(\mathrm{mmHg})\end{array}$ & $81.0(7.6)$ & $80.6(7.0)$ & $81.2(7.1)$ & $81.1(6.6)$ \\
\hline & HR (BPM) & $78.2(18.2)$ & $77.8(17.1)$ & $77.2(16.7)$ & $78.2(16.0)$ \\
\hline & RAP & $0.57(0.26)$ & $0.61(0.22)$ & $0.65(0.20)$ & $0.66(0.19)$ \\
\hline & PRx & $0.13(0.24)$ & $0.11(0.23)$ & $0.09(0.24)$ & $0.06(0.23)$ \\
\hline & RAC & - 0.21 (0.29) & $-0.25(0.28)$ & - 0.28 (0.29) & $-0.29(0.28)$ \\
\hline & PAx & $0.00(0.25)$ & - $0.01(0.25)$ & - $0.02(0.26)$ & - $0.03(0.26)$ \\
\hline \multirow{4}{*}{ Impairement } & PRx & $0.07(0.08)$ & $0.07(0.07)$ & $0.07(0.06)$ & $0.06(0.06)$ \\
\hline & $\mathrm{PAx}$ & 0.07 (0.09) & $0.07(0.08)$ & $0.07(0.08)$ & $0.07(0.08)$ \\
\hline & RAC & $0.11(0.12)$ & $0.10(0.10)$ & $0.10(0.10)$ & $0.10(0.10)$ \\
\hline & ICP & $0.14(0.44)$ & $0.22(0.76)$ & $0.33(1.24)$ & $0.40(1.58)$ \\
\hline \multirow{3}{*}{ Slope } & $\begin{array}{l}\mathrm{PRx}(\mathrm{PRx} / \\
\min )\end{array}$ & $-0.18(1.20)$ & - $0.16(0.71)$ & - $0.20(0.47)$ & - $0.20(0.39)$ \\
\hline & $\begin{array}{l}\text { RAC (RAC/ } \\
\min )\end{array}$ & $-0.61(1.31)$ & - $0.29(0.65)$ & - $0.25(0.48)$ & $-0.22(0.36)$ \\
\hline & $\begin{array}{l}\mathrm{PAx}(\mathrm{PAx} / \\
\min )\end{array}$ & $-0.40(1.17)$ & - $0.12(0.55)$ & - $0.12(0.35)$ & $-0.14(0.30)$ \\
\hline
\end{tabular}

Table S.4.1: Average of the CRASH risk score and the mean of physiological values for each time segment. Noticeable is that the mean of cerebral autoregulatory parameters changes with different time periods and that the slope of RAC and PAx differs strongly in the oh- $6 \mathrm{~h}$ time period compared to the rest of the time periods. 


\subsubsection{Prediction of new patients}

To predict new patients as favourable or unfavourable, calculate the $\mathrm{Y}$ value as displayed in Equation 4.7.1 using the corresponding regression coefficients given in Table S4.2 and compare $\mathrm{Y}$ to the threshold given. If $\mathrm{Y}$ is equal to or higher than the corresponding threshold, the patient is predicted to have an unfavourable outcome. Otherwise, the patient is predicted to have a favourable outcome.

$Y=$ Intercept $+\beta_{1} * \operatorname{Par}_{1}+\beta_{2} * \operatorname{Par}_{2}+\cdots+\beta_{n} * \operatorname{Par}_{r_{1}}$

\begin{tabular}{lllll} 
& oh-6h & oh-12h & oh-18h & oh-24h \\
\hline Threshold & 1.259 & 1.166 & 0.136 & -1.506 \\
\hline Intercept & 19.3 & 2.78 & -6.96 & 4.66 \\
\hline $\begin{array}{l}\text { CRASH risk } \\
\text { score }\end{array}$ & 0.161 & 0.083 & 0.166 & 0.0885 \\
Mean ABP & -0.375 & -0.11 & -0.113 & -0.140 \\
\hline Mean PRx & & & 19.2 & \\
Mean PAx & & & -25.0 & \\
Slope of PRx & 2.13 & & & -4.16 \\
Slope of RAC & 2.46 & & 1.94 & 8.24 \\
$\begin{array}{l}\text { Slope of PAx } \\
\text { RAC }\end{array}$ & -4.96 & & & \\
impairment & & 15.2 & 44.4 & 25.2 \\
$\begin{array}{l}\text { PRx } \\
\text { impairement }\end{array}$ & & & &
\end{tabular}

Table S.4.2: Regression coefficients and threshold for each model. Only filled cells are used for calculation. A negative value indicates that an increase in that parameter corresponds to a lower probability of unfavourable outcome. 



\section{Chapter 5}

\section{A machine-learning based analysis for the recognition of progressive central hypovolemia}

Frank C. Bennis, Björn J. van der Ster, Johannes J. van Lieshout, Peter Andriessen, Tammo Delhaas

Physiol Meas. 2017;38(9):1791-1801. 


\begin{abstract}
Background

Traditional patient monitoring during surgery includes heart rate (HR), blood pressure (BP) and peripheral oxygen saturation. However, their use as predictors for central hypovolemia is limited, which may lead to cerebral hypoperfusion. The aim of this study was to develop a monitoring model that can indicate a decrease in central blood volume (CBV) at an early stage.
\end{abstract}

\title{
Methods
}

Twenty-eight healthy subjects (aged 18-50 years) were included. Lower body negative pressure $(-50 \mathrm{mmHg})$ was applied to induce central hypovolemia until the onset of presyncope. Ten beat-to-beat and four discrete parameters were measured, normalized, and filtered with a $30 \mathrm{~s}$ moving window. Time to pre-syncope was scaled from $100 \%-0 \%$. A total of 100 neural networks with $5,10,15,20$, or 25 neurons in their respective hidden layer were trained by $10,20,40,80,160$, or 320 iterations to predict time to pre-syncope for each subject. The network with the lowest average slope of a fitted line over all subjects was chosen as optimal.

\section{Results}

The optimal generalized model consisted of 10 hidden neurons, trained using 80 iterations. The slope of the fitted line on the average prediction was -0.64 (SD 0.35). The model recognizes in $75 \%$ of the subjects the need for intervention at $>200$ s before pre-syncope.

\section{Conclusion}

We developed a neural network based on a set of physiological variables, which indicates a decrease in CBV even in the absence of HR and BP changes. This should allow timely intervention and prevent the development of symptomatic cerebral hypoperfusion. 


\subsection{Introduction}

Anesthetized patients are prone to the development of undetected central hypovolemia, which is a shortage of blood volume directly available to the left ventricle of the heart. Central hypovolemia affects cardiac output (CO) and may jeopardize cerebral perfusion [1]. A decrease in central blood volume (CBV) may occur due to a variety of causes, ranging from bleeding and spinal anesthesia to preoperative fasting and the compression of major veins [2].

Traditional patient monitoring during surgery includes the monitoring of heart rate (HR), blood pressure (BP) and peripheral oxygen saturation. However, the value of these monitored parameters as an indicator for central hypovolemia is insufficient [3,4], as exemplified during World War II in which air raid victims suffered from major blood loss with relative bradycardia rather than the expected tachycardia [5]. Faced with central hypovolemia, cardiovascular control mechanisms maintain $\mathrm{BP}$ despite reductions in $\mathrm{CBV}$ of up to $30 \%$. A decrease beyond approximately $30 \%$ of the CBV marks the second phase of hypovolemic shock. In this phase, a Bezold-Jarish-like reflex ceases sympathetic activity together with the development of vagal activation [6-8]. As a result, HR and/or systemic vascular resistance (SVR) decline, followed by a decrease in BP [9-12]. The late development of changes in HR and BP explains the clinical delay in recognizing the occurrence of central hypovolemia. As a result, the development of cerebral hypoperfusion during surgery may pass unnoticed, resulting in brain hypoxemia with the risk of developing postoperative neurological impairment [13].

Although cardiovascular parameters such as thorax impedance (TI) and variation in pulse pressure $\left(\mathrm{P}_{\text {pulse }}\right)$ relate to $\mathrm{CBV}$ [14-18] and thus may contain valuable information related to central hypovolemia in an early stage, each is unable to indicate central hypovolemia by itself. However, combining several of these parameters may provide a better prediction. To interpret the complex relationship between numerous continuous cardiovascular parameters for early or even hidden information, machine learning may offer new opportunities for complex biomedical signal analysis to support perioperative monitoring. Among machine learning models, neural networks are used to create a prediction based on the complex and non-linear relationships between parameters [19]. We evaluated whether a neural network approach using a large set of biomedical parameters can add in diagnosing a decrease in CBV at an early stage. Therefore, the aim of this study was to develop a neural network using beatto-beat physiological parameters and static parameters (age, gender, weight, and height) to diagnose a decrease in CBV at an early stage. 


\subsection{Methods}

\subsubsection{Subject population}

Healthy, non-smoking subjects aged between 18 and 50 years were recruited by advertisement in the Academic Medical Centre in Amsterdam (AMC), excluding any subject with a history of and/or treatment for cardiovascular disease, diabetes, and fainting. The measurements were performed between 6 January 2015 and 11 June 2015. Prior to inclusion, a full medical history was obtained, followed by physical examination and oscillometric BP as well as ECG measurements to assess the presence of cardiovascular contra-indications for the procedure. The study was approved by the Medical Ethics Committee of the AMC. Written informed consent was obtained from all subjects before any measurement.

\subsubsection{Study design}

Measurements were performed in a quiet room. Subjects were informed about the procedures involved and instructed to empty their bladder prior to the start of testing. They abstained from coffee, tea, sports, and fatty foods the day before measurement. With the subject in the supine resting position a lower-body negative pressure (LBNP) box was placed over the lower body. The LBNP box sealed the subject from hip to toes, enabling the buildup of a pressure difference between the upper and lower body. It was verified whether the box created and maintained a subatmospheric pressure of $-50 \mathrm{mmHg}$. A baseline of $5 \mathrm{~min}$, consisting of the same parameters obtained during LBNP, was measured after $25 \mathrm{~min}$ of supine rest. A LBNP of $-50 \mathrm{mmHg}$ was induced after the baseline and maintained for $30 \mathrm{~min}$ or terminated earlier in the case of the development of signs or symptoms of pre-syncope such as lightheadedness, nausea, or blurry vision. Pre-syncope was additionally defined as a sudden consistent reduction of $>25 \mathrm{mmHg}$ in systolic blood pressure $\left(\mathrm{P}_{\text {sys }}\right)$, of $>15 \mathrm{mmHg}$ in diastolic blood pressure $\left(\mathrm{P}_{\text {dia }}\right)$, or of $>15$ beats/min in HR, as these parameters will change with a large ( $>30 \%$ ) decrease of $\mathrm{CBV}[7]$. The start and end of the baseline and LBNP was indicated by markers. Data from the subjects not experiencing pre-syncope within $30 \mathrm{~min}$ were excluded from the analysis.

\subsubsection{Measurement parameters}

BP was measured by plethysmography at the middle finger of the left hand (Nexfin, Edwards Lifesciences BMEYE, Amsterdam, The Netherlands) during the entire experiment. The signal was stored at $200 \mathrm{~Hz}$. TI (Model AI-601G, Nihon Kohden, Japan) was measured by placing electrodes in the neck and on the upper body at the level of the xiphoid process. TI measures conduction through the chest as a gauge of CBV. The analog TI signal and a marker device were sampled at $200 \mathrm{~Hz}$ and stored on disk. The raw BP curve was extracted from the Nexfin. All signals were resampled at $100 \mathrm{~Hz}$ using MATLAB (2016A, The MathWorks, Natick, MA, USA). Nine beat-to-beat parameters were extracted directly from the Nexfin. These parameters consist of $\mathrm{P}_{\text {sys }}, \mathrm{P}_{\text {dia }}$, mean arterial pressure $\left(\mathrm{P}_{\text {mean }}\right), \mathrm{P}_{\text {pulse }}, \mathrm{HR}, \mathrm{SV}, \mathrm{CO}, \mathrm{SVR}$, and left ventricular ejection time (LVET). Corresponding beat-to-beat values of TI were added as a parameter. Age, gender, height, and weight were entered as static parameters. 


\subsubsection{Analysis}

Analysis was performed off-line using a custom-made MATLAB algorithm combined with the neural network toolbox in MATLAB. The LBNP period was selected based on the manually defined markers. A $30 \mathrm{~s}$ baseline period containing few or no artifacts was selected out of the recorded baseline to accurately reflect the resting state of the subject. Recordings during the first $120 \mathrm{~s}$ of LBNP were removed to discard the sudden hemodynamic changes that occur at the start of LBNP. Data was visually inspected for artifacts in the raw BP curve and in the beat-to-beat parameters HR, SV, SVR, and TI. Artifacts were removed with subsequent interpolation of the remaining data.

All beat-to-beat parameters were normalized to their median value within the selected baseline period. Thereafter, these were smoothed using an averaging filter with a moving $30 \mathrm{~s}$ window. Because time to pre-syncope and HR varied between subjects, the amount of beatto-beat parameter values estimated for each subject differed between subjects. To ensure that each subject contributed an equal number of samples to the model, subject data were resampled to the median number of beat-to-beat parameter values over all subjects.

\section{Model design and statistics}

The input to the neural network for the prediction at the time point of each heartbeat contained ten beat-to-beat parameters measured at that single heartbeat, as well as static data on age, sex, height, and weight (Figure 5.1). Time to pre-syncope was scaled from 100\%o\%, with $100 \%$ defined as 120 s after initiation of LBNP and $0 \%$ as the onset of pre-syncope. Time to pre-syncope was the desired output of the model for each training instance.

Data was divided into a training set and test set. The training set consisted of all but one subject. The test set consisted of the remaining subject. Each subject served once as a test set. For each test set, 100 neural networks were trained using the training set, as more neural networks did not increase performance, while it did increase computation time. All neural networks were initialized randomly. Thereafter, models were trained using the LevenbergMarquardt algorithm for backpropagation. Each of the 100 neural networks predicted the time to pre-syncope for the selected test subject. These 100 predictions were averaged to create a single average prediction of the selected test subject. We assumed normovolemia at the initiation of LBNP and therefore the average predicted time to pre-syncope was set equal to $100 \%$ at the start. Each of the tested subjects served once as a test set (leave-one-out cross-validation) in the above-described procedure, finally resulting in an average prediction for each subject. These predictions ideally start at $100 \%$ when the true time remaining is also $100 \%$, and indicate $0 \%$ when the true time remaining is also $0 \%$. Therefore, when plotting the true time remaining versus the predicted time remaining, the ideal slope would be -1 .

The above-mentioned model was created with a variable number of nodes in the hidden layer and a variable number of iterations during the training phase (Figure 5.1), influencing the complexity of the model and the amount of training on the data. The number of hidden nodes were $5,10,15,20$, or 25 (Figure $5.1 \mathrm{~A}$ ) and the number of iterations 10, 20, 40, 80, 160 , or 320 (Figure 5.1B). This resulted in a total of 30 combinations of hidden neurons 

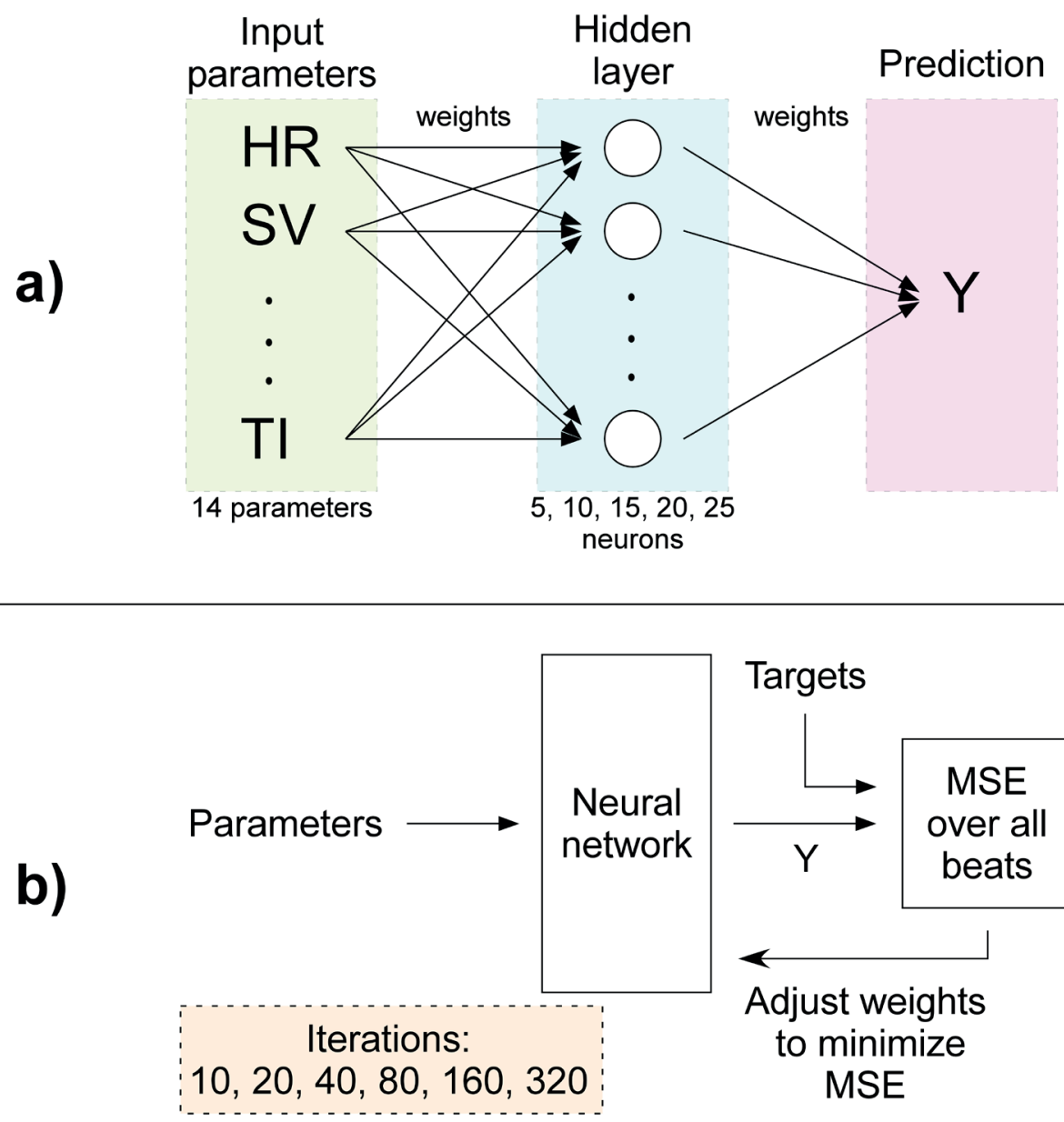

Targets

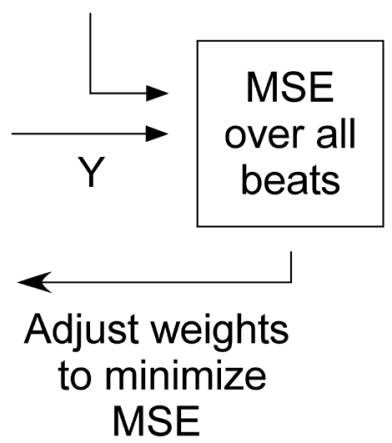

Figure 5.1: A Overview of the neural network. A total of 14 input parameters are connected by weights to the hidden layer with a variable number of neurons. The hidden layer neurons are connected by weights with the output (Y). B Overview of a single iteration of the training model. Parameters enter the neural network, which generate a certain output for each beat. The mean squared error (MSE) of all predicted outputs compared with the actual output is generated. After this, the weights are altered to minimize the MSE. 
and training iterations. Because each combination leads to an average prediction over 100 neural networks, the result was a grid of average predictions for each subject. A straight line was fitted to each of the 30 average predictions, the slope of which should be ideally -1 , i.e. equal to the expected target slope. For each combination of hidden nodes and number of iterations, the slopes were subsequently averaged over all subjects. Each averaged slope can be interpreted as the expected slope of the prediction on a new subject. The least complex combination of hidden nodes and iterations that led to a slope closest to -1 with the lowest standard deviation (SD) was considered the optimal model. Using the outcome on the test set to select the optimal model may introduce an optimistic outlook. However, no contamination between the training and test set is expected, because the data are already trained and tested at the moment of optimal model selection, thus reducing the possibility of an overly optimistic outlook. The fitted line on a prediction is used to evaluate the quality of the neural network, while the actual predictions by the model assess the quality of the model-prediction in an individual subject.

\subsection{Results}

A total of 28 subjects (12 male, aged $25 \pm 5$ years, weight $71 \pm 10 \mathrm{~kg}$, height $174 \pm 11 \mathrm{~cm}$ ) were included and experienced pre-syncope. The procedure was stopped because of a decline in BP $(n=2)$, symptoms such as dizziness, lightheaded and impaired vision $(n=10)$, or a combination of symptoms and a decline in BP or HR $(n=14)$. The reason for discontinuation of the procedure was not documented for two subjects.

The model with 80 iterations and ten hidden neurons was found to be the least complex model where the slope was closest to -1 with the lowest SD (Table 5.1). The average prediction with the SD of this model shows how the model would predict on average on new subjects (Figure 5.2). The median SD of the residuals of the prediction of each subject minus the corresponding fitted line is 7.2 , indicating that the predictions differ up to plus minus $7.2 \%$ from the fitted line in two-thirds of the predictions.

\begin{tabular}{|llllll}
\hline \multicolumn{7}{c}{ Neurons in the hidden layer } \\
Iterations & $\mathbf{5}$ & $\mathbf{1 0}$ & $\mathbf{1 5}$ & $\mathbf{2 0}$ & $\mathbf{2 5}$ \\
\hline 10 & $-0.49(0.24)$ & $-0.58(0.30)$ & $-0.60(0.32)$ & $-0.60(0.34)$ & $-0.61(0.36)$ \\
\hline 20 & $-0.55(0.28)$ & $-0.62(0.34)$ & $-0.61(0.37)$ & $-0.62(0.38)$ & $-0.62(0.41)$ \\
\hline 40 & $-0.56(0.29)$ & $-0.62(0.36)$ & $-0.62(0.37)$ & $-0.64(0.40)$ & $-0.63(0.44)$ \\
\hline 80 & $-0.57(0.28)$ & $-0.64(0.35)$ & $-0.61(0.35)$ & $-0.62(0.37)$ & $-0.62(0.44)$ \\
\hline 160 & $-0.57(0.28)$ & $-0.64(0.35)$ & $-0.59(0.36)$ & $-0.58(0.34)$ & $-0.57(0.41)$ \\
\hline 320 & $-0.57(0.28)$ & $-0.64(0.35)$ & $-0.59(0.35)$ & $-0.59(0.34)$ & $-0.57(0.37)$ \\
\hline
\end{tabular}

Table 5.1: Mean slopes with standard deviation for each combination of iterations and number of neurons in the hidden layer. Four models have a slope of -0.64, which is the closest to the target slope of -1. The least complex model with the lowest standard deviation is displayed in bold. 


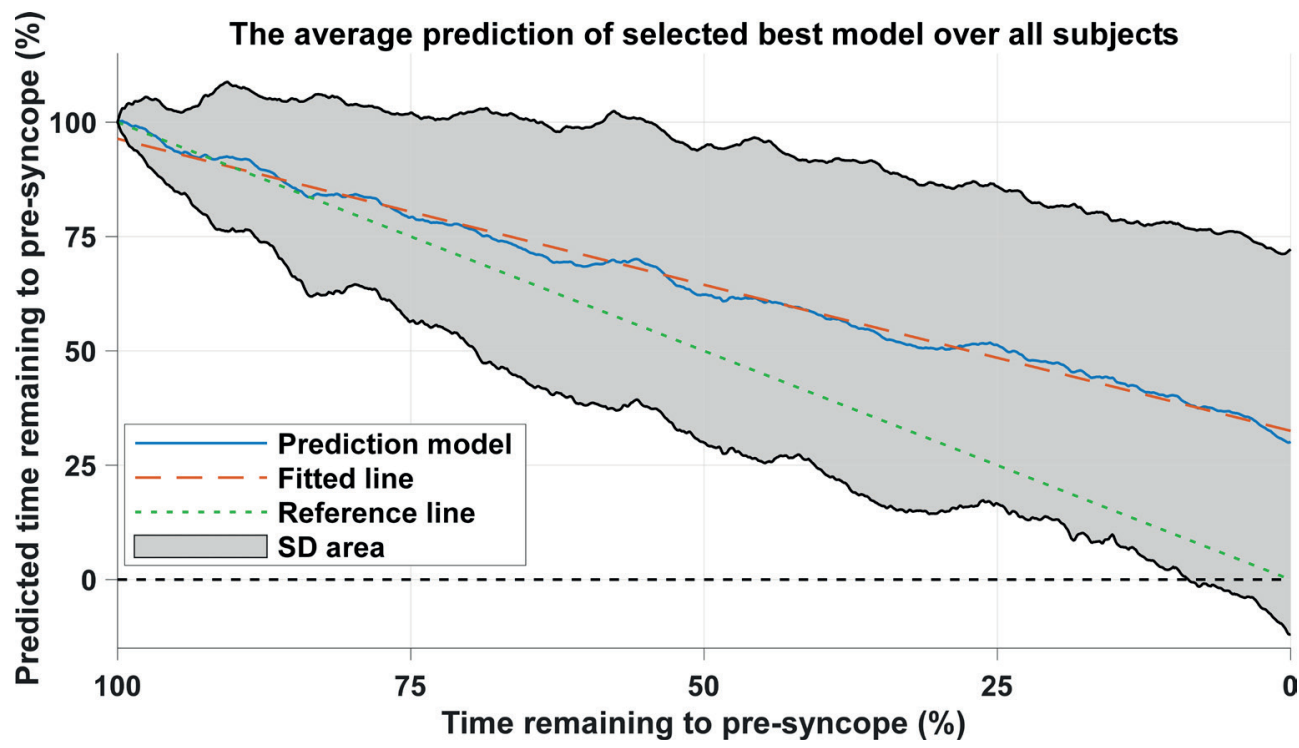

Figure 5.2: The average prediction of the selected best model on the test subjects. At $100 \%$ the test starts, at $0 \%$ pre-syncope occurs. This prediction shows how the model would predict on average on new subjects. The average prediction shows a stable decline with a slope less steep than the actual slope.
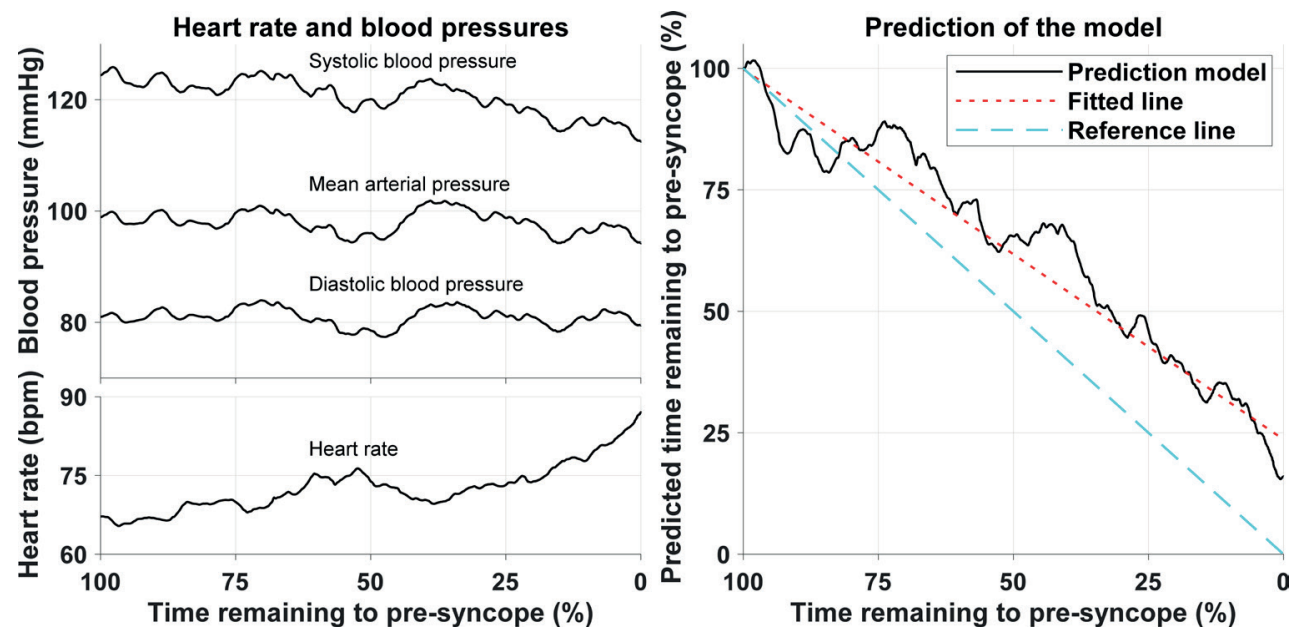

Figure 5.3: Left: Commonly measured parameters $\mathrm{P}_{\mathrm{syy}}, \mathrm{P}_{\text {dia }}$, Pmean and HR. Blood pressures remain stable, while HR shows a clear increase the moment less than $25 \%$ of time is remaining. Right: The predicted time remaining to pre-syncope in \% for this subject. A decreasing trend for CBV can be seen from the beginning of the experiment. 
The prediction of the model was compared to the evolution of BP and HR level of an individual (Figure 5.3). The model indicated a decrease in time remaining to pre-syncope from the start of LBNP with a slope of 0.76 for this particular subject, whereas the parameters BP and $\mathrm{HR}$, respectively, did not show any change or changed in a later phase. A decrease in time remaining to pre-syncope indicated by the model was not just seen for this subject but for each subject, as the fitted line on the prediction displayed a negative slope for each subject (not shown).

Ideally (slope $=-1$ ), the model predicts $0 \%$ time remaining to pre-syncope with o s remaining. If the model-predicted slope is steeper than in reality $(<-1)$, the model predicts $0 \%$ time remaining to pre-syncope, while in reality there is time remaining to pre-syncope. If the model-predicted slope is less steep than in reality $(>-1)$, the model does not reach $0 \%$ before pre-syncope occurs, resulting in an overestimation of the remaining part. In this study, the model predicted $0 \%$ time remaining to pre-syncope for six subjects, with on average $253 \mathrm{~s}$ remaining (Figure 5.4). For a total of 14 subjects, the model predicted $25 \%$ time remaining to pre-syncope, with on average $225 \mathrm{~s}$ remaining. The $50 \%$ time remaining to pre-syncope level is predicted for 21 subjects, with on average $342 \mathrm{~s}$ remaining, while for all but one subject the model predicted $75 \%$ time remaining to pre-syncope with on average $439 \mathrm{~s}$ remaining. If we assume intervention is needed when predicted time remaining is $\leq 75 \%$, the model recognizes the need for intervention more than $200 \mathrm{~s}$ before pre-syncope in $75 \%(n=21)$ of the subjects.

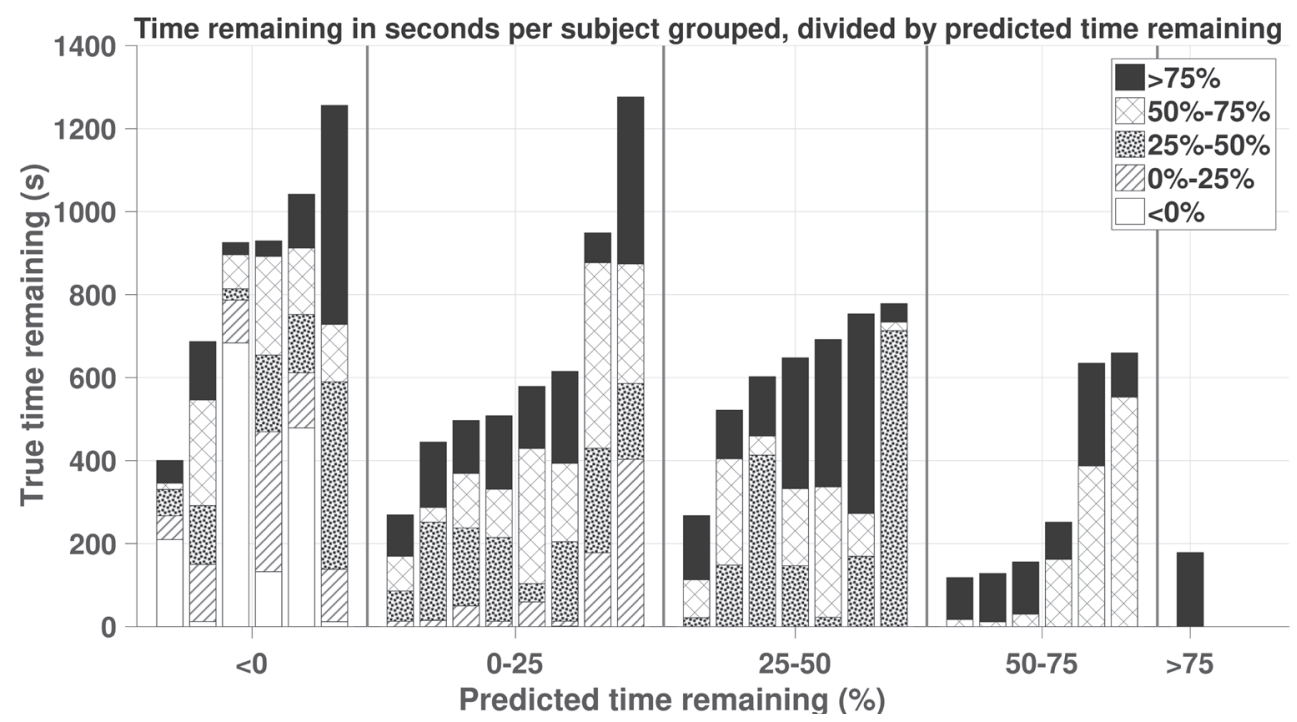

Figure 5.4: Total time in seconds remaining to pre-syncope vs the predicted time remaining per subject. Subjects are divided into groups based on the lowest value of time remaining predicted by the model. Lower predicted times remaining at o seconds remaining reflect a steeper slope, with a predicted time remaining of $0 \%$ as ideal. Each bar represents one subject, of which the top corresponds to a predicted time of $100 \%$ with the amount of seconds remaining as displayed on the y-axis. As the subject progresses in time, the true time remaining in seconds decreases, while the predicted time remaining also decreases. This is visualized by the different segments in one bar. 


\subsection{Discussion}

The created neural network recognized a decrease in CBV as evoked by LBNP in an early stage using beat-to-beat cardiovascular parameters and information on age, weight, height, and sex. In individual patients, the measured downward trend may be seen before BP and HR show any noticeable changes (Figure 5.3). As the procedure was discontinued in ten subjects, due to symptoms indicated by the subject, while during anesthesia the subject is not able to indicate these symptoms, pre-syncope as a serious expression of central hypovolemia will not be recognized until in a later stage.

As soon as a decrease in BP or HR was recognized, the procedure was discontinued. We showed that our model indicated a decrease well before discontinuation of the procedure (Figure 5.4) and thus before a decrease in BP or HR (Figure 5.3), with $>200$ s remaining in $75 \%$ of the subjects at the $75 \%$ level. The SD of the residuals shows that while fluctuations are present, the prediction is on average relatively stable compared to the fitted line. It has to be noted that of the seven subjects in which prediction did not reach $75 \%$ with more than $200 \mathrm{~s}$ remaining, the time spent in LBNP without the initial $120 \mathrm{~s}$ was $<200 \mathrm{~s}$ for four persons and between 200 and $300 \mathrm{~s}$ for the remaining three. It is possible that the procedure was stopped too early in these cases, due to false symptom recognition. If we exclude subjects in whom pre-syncope occurred before $300 \mathrm{~s}$, the model reaches the $75 \%$ threshold for every subject and $50 \%$ for 19 out of 21 subjects. These results indicate that because the model can recognize a decrease in CBV in an early stage, it may assist the anesthesiologist in decision-making. Starting treatment for central hypovolemia at low values of predicted time remaining results in treating few subjects, but may prevent pulmonary and/or peripheral edema due to overfilling. The model was able to detect pending pre-syncope and subsequent cerebral hypoperfusion, while none of the parameters used measured cerebral perfusion. Incorporating parameters that measure cerebral perfusion may further improve the recognition of decreased CBV. The two commonly used parameters to measure cerebral perfusion, near-infrared spectroscopy and transcranial Doppler ultrasound, may both be able to show changes in cerebral perfusion before other hemodynamic parameters [20,21].

\subsubsection{Comparison with literature}

Machine learning techniques were also used to develop the compensatory reserve index (CRI), indicating the amount of reserve before pre-syncope will occur in a subject on a scale from one to zero [22,23]. The CRI is shown to be more predictive for hypovolemia than the commonly used shock index, which is the ratio of HR to $\mathrm{P}_{\text {sys }}$ [24]. Furthermore, a decrease in CRI is seen when a small amount of blood $(450 \mathrm{ml})$ is withdrawn from a subject $[25,26]$. However, when withdrawing an average of $1.2 \mathrm{l}$ in 20 subjects, the CRI decreased on average by $33 \%$. This small decrease in CRI suggests that the CRI underestimates larger amounts of blood loss [27].

An important difference between our model and the CRI is the collected data on which to train the respective models. The CRI model is trained on subjects who underwent a stepwise LBNP protocol in which LBNP is lowered after every $5 \mathrm{~min}$ by $5 \mathrm{mmHg}$, until pre-syncope occurs. The CRI is based on the prediction of the current level of LBNP and the predicted level 
of LBNP at which pre-syncope will occur. During a 5 min period at a certain level of LBNP, both the predicted current and final level of LBNP will be constant if the algorithm is correct. Therefore, during this $5 \mathrm{~min}$ period, the CRI will be stable. This is also seen in the outcome, as the predicted levels of LBNP show a stepwise fashion, with high correlation to the actual level of LBNP $(r=0.94)$ [22]. When estimating the CRI out of these levels of LBNP, the result is a stepwise CRI [23]. However, even though LBNP is constant between two steps, there is still a change in CBV, which is not taken into account by the CRI. In our study, a constant level of LBNP was used, resulting in a continuous change in CBV during the procedure. Therefore, our model is trained to detect a change in CBV without the effect of the change in LBNP, which may be closer to the real-life situation of central hypovolemia. Furthermore, our study is to be applied in the operating room (OR). As the CRI is developed for the battlefield, our model can take more parameters into account. The difference in the model as well as the target group makes this a good addition for use in the OR.

\subsubsection{Limitations}

First, the number of subjects in our neural network is relatively small. Although this drawback is partially compensated for by the low number of parameters, increasing the number of subjects may increase the accuracy of the prediction. Furthermore, increasing the number of subjects opens the possibility of creating more diversity in the models by subset selection of the subjects, leading to possible better predictions. Due to the low number of subjects, no validation set was used in this study. This may introduce an overly optimistic outlook for the selected optimal model. In this study, however, a clear downward trend was found for all possible combinations of hidden neurons and iterations (Table 5.1). This suggests that a good prediction model would have been found for all possible combinations tested. For future studies, the inclusion of a validation set together with more subjects is recommended. Second, measurements were discontinued if the vasovagal response developed or if a subject indicated symptoms of pre-syncope. Because the onset of symptom occurrence is not by definition equal to the moment of pre-syncope, some measurements might have been interrupted early. Although this fact warrants subgroup analysis based on stopping criteria, this cannot be performed due to the low number of subjects. Third, we set the average prediction at the start equal to $100 \%$, because we assumed normovolemia at the initiation of LBNP. It is realistic that the subjects were slightly hypovolemic, setting the true initial value below $100 \%$. This decreases the slope of the reference line and may indicate that the calculated slope for the subjects may be closer to the reference slope than stated previously. Finally, the model requires measurement of a baseline period, which may not be available in acute cases. In these cases, baseline values may be estimated based on patient characteristics. A different approach would be to abandon normalization of parameters to the baseline, though possibly at the expense of prediction accuracy. The possibly lower prediction accuracy when using non-normalized parameters might be counteracted by the inclusion of trend parameters.

\subsection{Conclusion}

We developed a neural network based on a set of physiological variables that can diagnose decreases in CBV at an early stage even in the absence of HR and BP changes. This should allow for more timely intervention and may prevent cerebral hypoperfusion. 


\subsection{References}

1. Van Lieshout JJ, Wieling W, Karemaker JM, Secher NH. Syncope, cerebral perfusion, and oxygenation. J Appl Physiol. 2003;94(3):833-48.

2. Joshi G. Intraoperative fluid management. 2016 [cited 2017 Jan 12]. Available from: https://www.uptodate.com/contents/intraoperative-fluid-management

3. Hamilton-Davies C, Mythen MG, Salmon JB, Jacobson D, Shukla A, Webb AR. Comparison of commonly used clinical indicators of hypovolaemia with gastrointestinal tonometry. Intensive Care Med. 1997;23(3):276-81.

4. Orlinsky M, Shoemaker W, Reis ED, Kerstein MD. Current controversies in shock and resuscitation. Surg Clin North Am. 2001;81(6):1217-62.

5. Grant RT, Reeve EB. Clinical Observations on Air-raid Casualties. Br Med J. 1941;2(4208):293-7.

6. Kinsella SM, Tuckey JP. Perioperative bradycardia and asystole: relationship to vasovagal syncope and the Bezold-Jarisch reflex. Br J Anaesth. 2001;86(6):859-68.

7. Secher NH, van Lieshout JJ. Hypovolemic Shock. In: Clinical Fluid Therapy in the Perioperative Setting. Cambridge: Cambridge University Press; 2011. p. 166-76.

8. Jarisch A, Richter H. Der Bezold-Effekt - Eine Vergessene Kreislaufreaktion. Klin Wochenschr 1939;18(6):185-7.

9. Schadt JC, Ludbrook J. Hemodynamic and neurohumoral responses to acute hypovolemia in conscious mammals. Am J Physiol. 1991;260(2):H305-18.

10. Barcroft H, Edholm OG. On the vasodilatation in human skeletal muscle during posthaemorrhagic fainting. J Physiol. 1945;104(2):161-75.

11. Warren J V, Brannon ES, Stead EA, Merrill AJ. The effect of venesection and the pooling of blood in the extremities on the atrial pressure and cardiac output in normal subjects with observations on acute circulatory collapse in three instances. J Clin Invest. 1945;24(3):337-44.

12. van Lieshout JJ, Wieling W, Karemaker JM, Eckberg DL. The vasovagal response. Clin Sci (Lond). 1991;81(5):575-86.

13. Casati A, Fanelli G, Pietropaoli P, Proietti R, Tufano R, Danelli G, et al. Continuous monitoring of cerebral oxygen saturation in elderly patients undergoing major abdominal surgery minimizes brain exposure to potential hypoxia. Anesth Analg. 2005;101(3):740-7.

14. van Lieshout JJ, Harms MPM, Pott F, Jenstrup M, Secher NH. Stroke volume of the heart and thoracic fluid content during head-up and head-down tilt in humans. Acta Anaesthesiol Scand. 2005;49(9):1287-92.

15. Cai Y, Holm S, Jenstrup M, Strømstad M, Eigtved A, Warberg J, et al. Electrical admittance for filling of the heart during lower body negative pressure in humans. $\mathrm{J}$ Appl Physiol. 2000;89(4):1569-76.

16. Convertino VA, Cooke WH, Holcomb JB. Arterial pulse pressure and its association with reduced stroke volume during progressive central hypovolemia. J Trauma. 2006;61(3):629-34.

17. Bronzwaer A-SGT, Ouweneel DM, Stok WJ, Westerhof BE, van Lieshout JJ. Arterial Pressure Variation as a Biomarker of Preload Dependency in Spontaneously Breathing Subjects - A Proof of Principle. PLoS One. 2015;10(9):e0137364. 
18. Bronzwaer A-SGT, Stok WJ, Westerhof BE, van Lieshout JJ. Arterial pressure variations as parameters of brain perfusion in response to central blood volume depletion and repletion. Front Physiol. 2014;5:157.

19. Mitchell T. Artificial Neural Networks. In: Machine Learning. New York: McGraw-Hill; 1997. p. 81-127.

20. Plachky J, Hofer S, Volkmann M, Martin E, Bardenheuer HJ, Weigand MA. Regional cerebral oxygen saturation is a sensitive marker of cerebral hypoperfusion during orthotopic liver transplantation. Anesth Analg. 2004;99(2):344-9.

21. Albina G, Fernandez Cisneros L, Laiño R, Nobo UL, Ortega D, Schwarz E, et al. Transcranial Doppler monitoring during head upright tilt table testing in patients with suspected neurocardiogenic syncope. EP Eur. 2004;6(1):63-9.

22. Convertino VA, Moulton SL, Grudic GZ, Rickards CA, Hinojosa-Laborde C, Gerhardt RT, et al. Use of advanced machine-learning techniques for noninvasive monitoring of hemorrhage. J Trauma. 2011;71(1):S25-32.

23. Moulton SL, Mulligan J, Grudic GZ, Convertino VA. Running on empty? The compensatory reserve index. J Trauma Acute Care Surg. 2013;75(6):1053-9.

24. Van Sickle C, Schafer K, Mulligan J, Grudic GZ, Moulton SL, Convertino VA. A sensitive shock index for real-time patient assessment during simulated hemorrhage. Aviat Space Environ Med. 2013;84(9):907-12.

25. Stewart CL, Mulligan J, Grudic GZ, Convertino VA, Moulton SL. Detection of lowvolume blood loss: compensatory reserve versus traditional vital signs. J Trauma Acute Care Surg. 2014;77(6):892-8.

26. Nadler R, Convertino VA, Gendler S, Lending G, Lipsky AM, Cardin S, et al. The value of noninvasive measurement of the compensatory reserve index in monitoring and triage of patients experiencing minimal blood loss. Shock. 2014;42(2):93-8.

27. Convertino VA, Howard JT, Hinojosa-Laborde C, Cardin S, Batchelder P, Mulligan J, et al. Individual-Specific, Beat-to-beat Trending of Significant Human Blood Loss: The Compensatory Reserve. Shock. 2015;44 Suppl 1:27-32. 



\section{Chapter 6}

\section{Support vector machine based monitoring of cardio-cerebrovascular reserve during simulated hemorrhage}

Björn J. van der Ster, Frank C. Bennis, Tammo Delhaas, Berend E. Westerhof, Wim J. Stok, Johannes J. van Lieshout

Front Physiol. 2018;8:1057 


\begin{abstract}

\section{Background}

In the initial phase of hypovolemic shock, mean blood pressure (BP) is maintained by sympathetically mediated vasoconstriction rendering BP monitoring insensitive to detect blood loss early. Late detection can result in reduced tissue oxygenation and eventually cellular death. We hypothesized that a machine learning algorithm that interprets currently used and new hemodynamic parameters could facilitate in the detection of impending hypovolemic shock.
\end{abstract}

\title{
Methods
}

In 42 (27 female) young (mean (SD): 24 (4) years), healthy subjects central blood volume (CBV) was progressively reduced by application of $-50 \mathrm{mmHg}$ lower body negative pressure until the onset of pre-syncope. A support vector machine was trained to classify samples into normovolemia (class o), initial phase of CBV reduction (class 1) or advanced CBV reduction (class 2). Nine models making use of different features were computed to compare sensitivity and specificity of different non-invasive hemodynamic derived signals. Model features included: volumetric hemodynamic parameters (stroke volume and cardiac output), BP curve dynamics, near-infrared spectroscopy determined cortical brain oxygenation, end-tidal carbon dioxide pressure, thoracic bio-impedance, and middle cerebral artery transcranial Doppler (TCD) blood flow velocity. Model performance was tested by quantifying the predictions with three methods: sensitivity and specificity, absolute error, and quantification of the log odds ratio of class 2 vs. class o probability estimates.

\section{Results}

The combination with maximal sensitivity and specificity for classes 1 and 2 was found for the model comprising volumetric features (class 1: $0.73-0.98$ and class 2: 0.56-0.96). Overall lowest model error was found for the models comprising TCD curve hemodynamics. Using probability estimates the best combination of sensitivity for class 1 (0.67) and specificity (o.87) was found for the model that contained the TCD cerebral blood flow velocity derived pulse height. The highest combination for class 2 was found for the model with the volumetric features (0.72 and 0.91).

\section{Conclusion}

The most sensitive models for the detection of advanced CBV reduction comprised data that describe features from volumetric parameters and from cerebral blood flow velocity hemodynamics. In a validated model of hemorrhage in humans these parameters provide the best indication of the progression of central hypovolemia. 


\subsection{Introduction}

Hypovolemic shock is the hemodynamic response to a critically reduced central blood volume (CBV) and its diagnosis has challenged clinicians since the Second World War [1,2]. The main treatment consists of intravenous volume administration [3] to raise cardiac output (CO) and improve microvascular blood flow $[2,4,5]$ and tissue oxygen delivery $[6,7]$. However, detection of a clinically relevant blood volume deficit remains difficult $[2,4,8,9]$ because the blood volume is not only characterized by its magnitude but also by its function as preload to the heart $[2,8,9]$. From that perspective, a functional definition of "normovolemia" is by its ability to provide the heart with an adequate $\mathrm{CBV}$ i.e. cardiac preload that maintains stroke volume, cardiac output, and oxygen delivery [10,11]. Direct measures of CBV are not routinely available in the clinical environments of intensive care and operating room. As a result, volume treatment during anesthesia is generally planned according to a somewhat arbitrary fixed volume regime [12] or guided by blood pressure (BP) and heart rate (HR). However, interpretation of $\mathrm{BP}$ and $\mathrm{HR}$ changes in response to a reduction in $\mathrm{CBV}$ is not straightforward since loss of one liter of blood or fluid is not reflected in changes in BP [13]. Therefore, optimization of tissue oxygen delivery cannot be conducted by monitoring arterial pressure alone [14-17]. It is problematic that present hemodynamic monitoring techniques do not allow detection and therefore early treatment of a volume deficit before worsening of the cardio-cerebrovascular condition compromising oxygenation of the brain [3].

We hypothesized that the arterial pressure and transcranial cerebral blood flow velocity waveforms contain subtle information on the actual cardio-cerebrovascular condition that is hard to interpret by human visual inspection. We set out to investigate whether a machine learning model [18] could be trained to detect hypovolemia using hemodynamic signals during progressive reduction of CBV. This would allow determination to what extent the cardiovascular system can compensate hypovolemia, i.e. its compensatory reserve prior to (impending) circulatory collapse [19], by classifying patients according to their actual need of fluid therapy [20] and allow timely clinical intervention. Given that the brain is highly susceptible to hypoperfusion and hypoxia we hypothesized that the cerebral flow velocity wave shape may disclose early alterations that can be alleged to the hypovolemia induced onset of cerebral hypoperfusion resulting in pre-syncope. Earlier machine learning approaches based on BP waveforms [21] and beat-to-beat parameters [22] showed that it can detect a reduction in $\mathrm{CBV}$. To that purpose, we parametrized both the $\mathrm{BP}$ and TCD waveforms to make information about curve dynamics available for statistical modeling during progressive hemorrhagic shock and compared the BP features to features from other non-invasive hemodynamic technologies. We trained a model to recognize progressive hypovolemia by means of supervised machine learning and tested it on a human model of progressive hemorrhagic shock (lower body negative pressure, LBNP). The goal was to create a model that picks up on changing physiology during the transitional phase from compensated to uncompensated circulatory shock by classifying each heartbeat based on its accompanying feature information and to check which non-invasive hemodynamic monitor contributes the most sensitive information to solve this problem. 


\subsection{Methods}

\subsubsection{Subjects}

Forty-two young, healthy volunteers (27 female; age: mean (SD): 24 (4) years) with no history of fainting and/or cardiac arrhythmia nor taking cardiovascular medication participated in the study. They abstained from heavy exercise and caffeinated beverages at least $12 \mathrm{~h}$ prior to the experiment. Before inclusion subjects underwent a medical screening prior to the experiment consisting of a medical interview, a physical examination and a 12-lead ECG. The experiments were conducted in a quiet, temperature-controlled laboratory $\left(20-22^{\circ} \mathrm{C}\right)$. This study was carried out in accordance with the recommendations of Academic Medical Centre Amsterdam medical ethical committee (\#2014_310) with written informed consent from all subjects. All subjects gave written informed consent in accordance with the Declaration of Helsinki. The protocol was approved by the medical ethical committee of the Academic Medical Centre, Amsterdam.

\subsubsection{Experimental protocol}

Measurementswereperformed with subjectsinthesupineposition. Followinginstrumentation, the lower body was positioned inside a LBNP box (Dr. Kaiser Medizintechnik, Bad Hersfeld, Germany) and sealed at the level of the iliac crest [23]. To prevent a downward shift of the body into the LBNP box disrupting the airtight sealing with loss of sub-atmospheric pressure, the LBNP box was equipped with a saddle [24]. Subjects rested for $30 \mathrm{~min}$ of which the final ten were designated as baseline segment, followed by application of a single step continuous negative pressure ( $50 \mathrm{mmHg}$ below atmospheric pressure) to the lower part of the body. The pressure inside the box was manually controlled and established within less than $20 \mathrm{~s}$.

During the experiment, subjects were instructed to breathe normally and to avoid movement and muscle flexing. In compliance with our laboratory safety guidelines LBNP was terminated in case of (pre-)syncopal symptoms including sweating, light-headedness, nausea, blurred vision, and/or signs meeting one or more of the following criteria: systolic arterial pressure $\left(\mathrm{P}_{\text {sys }}\right.$ below $80 \mathrm{mmHg}$, or rapid drop in $\mathrm{BP}\left(\mathrm{P}_{\text {sys }}\right.$ by $\geq 25 \mathrm{mmHg} \cdot \mathrm{min}-1$, diastolic arterial pressure $\left(\mathrm{P}_{\mathrm{dia}}\right)$ by $\left.\geq 15 \mathrm{mmHg} \cdot \mathrm{min}-1\right)$, and drop in $\mathrm{HR}$ by $\geq 15 \mathrm{bpm} \cdot \mathrm{min}-1$. If none of these criteria occurred within $30 \mathrm{~min}$, the protocol was ended. The subjects were continuously monitored by an investigator experienced in human studies and unoccupied by experimental obligations.

\subsubsection{Measurements}

Continuous arterial BP was measured non-invasively by volume-clamp finger plethysmography with the cuff placed around the middle phalanx of the left hand placed at heart level (Nexfin, Edwards Lifesciences BMEYE, Amsterdam, The Netherlands) and sampled at $200 \mathrm{~Hz}$. Left ventricular stroke volume (SV) was determined by a pulse contour method (Nexfin CO-trek, Edwards Lifesciences BMEYE, Amsterdam, the Netherlands). Cardiac output (CO) was calculated as the SV times HR and total peripheral resistance (TPR) 
was the ratio of mean arterial pressure $\left(\mathrm{P}_{\text {mean }}\right)$ to CO. Changes in CBV were monitored using thoracic impedance (TI) (Model AI-601G, Nihon Kohden, Japan) [25,26]. Cerebral blood flow velocity was measured in the proximal segment of the middle cerebral artery (MCA) by means of TCD (DWL Multidop X4, Sipplingen, Germany). The left MCA was insonated through the temporal window just above the zygomatic arch at a depth of 40-60 mm with a pulsed $2 \mathrm{MHz}$ probe. After signal optimization, the probe was fixed on a specially designed head-band (Marc 6oo, Spencer Technologies, Redmond, Washington, USA). Changes in oxygenated and deoxygenated hemoglobin $(\mathrm{Hb})$ as well as their summation were measured using continuous wave near-infrared spectroscopy (NIRS) (Oxymon, Artinis, Zetten, The Netherlands). NIRS tracks cortical cerebral oxygenation during manipulation of CBF in parallel with the brain capillary oxygen saturation [27]. A differential path length factor was computed according to Gersten et al. [28] to account for the scattering of light in the brain tissue. NIRS signals were recorded at $10 \mathrm{~Hz}$. Optodes were fixed just above the supraorbital ridge and below the hairline. Changes in cutaneous perfusion may interfere with the accuracy of cerebral oximetry, therefore the distance between the transmitter and the receivers was $5 \mathrm{~cm}$ to assure deep enough penetration of the near-infrared light into the brain to exclude substantial contamination from the extra-cerebral circulation [29].

End-tidal $\mathrm{CO}_{2}$ partial pressure $\left(\mathrm{ETCO}_{2}\right)$ was measured through a nasal cannula connected to a capnograph (Hewlett Packard 7834A, Wokingham, UK). All recorded signals were analyzed offline (MATLAB R2Oo7b, The MathWorks, Natick, MA, USA) and visually inspected for artifacts and noise. Invalid beats were manually removed and interpolated.

\subsubsection{Modeling approach}

Models were created by means of a support vector machine algorithm (libsum software package for MATLAB [30]). We used a supervised learning approach to detect worsening of the cardio-cerebrovascular condition from cardiovascular stability at rest toward instability when approaching pre-syncope. To this extent, we defined three distinct classes of the hemodynamic condition (see "class definition"). The algorithm then used one of nine designated feature sets (listed next) to detect patterns in an attempt to classify each heartbeat in one of the three classes. For each feature set a model was computed using a non-linear radial basis function (Gaussian) kernel [31]. To find the optimal model configuration for each respective feature set we used 64 combinations of values for both kernel width (gamma) and $\mathrm{C}$-value (8 values for each). Using a randomly selected 30 subjects train vs. 1 test subject approach, this analysis was deemed optimal once the sum of sensitivity and specificity was maximal on average for all tested subjects.

\section{Class definition}

Baseline rest as well as onset of LBNP and pre-syncope were marked. Time points originating from data during baseline were designated as class o, samples from data during the first $75 \%$ of LBNP as class 1 and samples belonging to the last 25\% of LBNP before onset of presyncope (i.e. end-stage LBNP) were defined as class 2 (Figure 6.1). Multiclass in libsvm is handled by a one-vs.-one approach [32]. The corresponding feature values at these time points were labeled with one of these three classes. Static features were extracted on a beat- 
to-beat basis whereas dynamic features (variation and trends over time) were extracted by a moving windowing function of fixed size (see model specifications) where each moved window was classified as one of three classes. Due to how the class definitions were created, class distribution was not homogenous. Around 33\% of the dataset was baseline data (class o); $50 \%$ was class 1 and $18 \%$ class 2

\section{Feature extraction}

To test the viability of different measured parameters from non-invasive measurement modalities we designed 7 models (named model \#1 through \#7). All shared the BP curve dynamics features (model \#1, Figure 6.2, Supplementary Table S6.1). Features were then appended for models \#2 through \#7 for each investigated measurement modality to evaluate predictive capability when adding features from $\mathrm{ETCO}_{2}$, TI, NIRS, or TCD in modeling impending pre-syncope. All extracted features were down sampled by a factor 10 to abridge calculation time. Two models (named models \#8 and \#9) stand on their own and do not include the BP curve dynamics feature set.

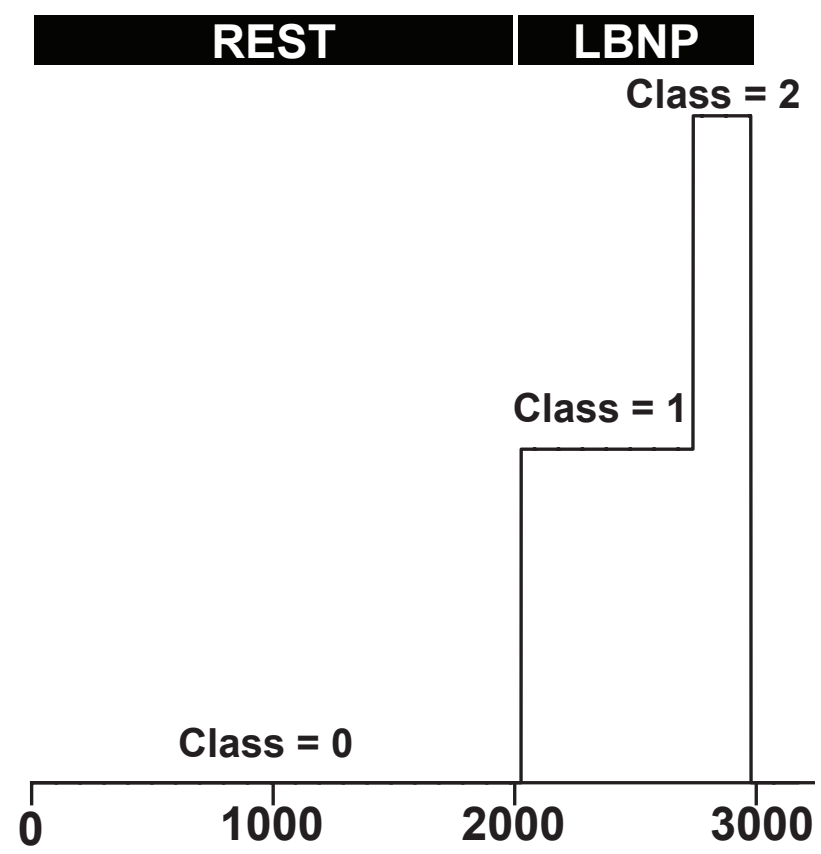

Figure 6.1: Class definitions. The first part of the measurement is defined as baseline rest (class o), LBNP is defined as class 1 , of which the last $25 \%$ is defined as end-stage LBNP before pre-syncope (class 2). 


\subsubsection{Default model: BP curve dynamics (model \#1)}

From the arterial BP wave, beat-to-beat systolic, diastolic, mean, pulse pressure $\left(\mathrm{P}_{\mathrm{sys}}, \mathrm{P}_{\text {dia }}\right.$, $\mathrm{P}_{\text {mean }}$, and $\mathrm{P}_{\text {pulse }}$ ), interbeat interval (IBI), HR, SV, CO, left ventricular ejection time (LVET), and TPR were extracted (10 features). Four incrementally sized intervals during LBNP (30, 60,90 , and $120 \mathrm{~s}$ ) were used for calculating trends and variances of $\mathrm{P}_{\text {sys }}, \mathrm{P}_{\text {dia }}, \mathrm{HR}, \mathrm{P}_{\text {pulse }}$, and SV (4 intervals times 5 parameters for 2 techniques (trend and variation) delivers 40 features). Additional information from the BP wave shape was extracted by wave segmentation and parametrization (Figure 6.2, Supplementary Table S6.1, 15 features) making a total of 65 parameters available for the BP curve dynamics model.

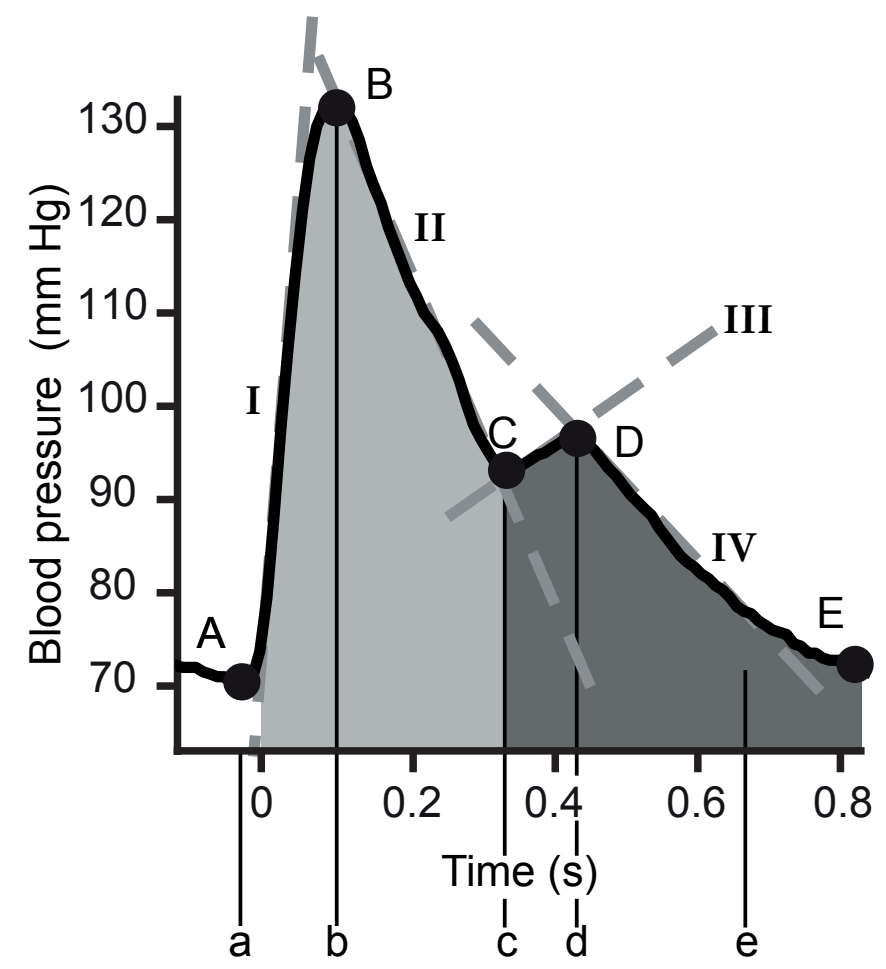

Figure 6.2: In depth analysis of the blood pressure curve. Five primary points are detected (A to E). From these points several parameters are estimated (Supplementary Table S6.1). Positions on the curve are indicated with capital letters A through E. Their accompanying time points are described with lower case letters. Tangent lines are described with roman numerals. Areas of interest are shaded. 


\subsubsection{Incremental models}

Either beat-to-beat interpolated $\mathrm{ETCO}_{2}$ partial pressure or TI were appended in models \#2 and \#3 respectively (each has 1 additional feature). Features extracted from the NIRS consisted of the three concentrations of $\mathrm{Hb}$ : oxygenated, deoxygenated, and their summation (total $\mathrm{Hb}$ ). Ratios of oxygenated and deoxygenated to total $\mathrm{Hb}$ were added as well to this model (model \#4, 5 additional features).

Similar to the BP wave parametrization, the same points, durations, tangents, and surface areas were derived from the cerebral blood flow velocity wave. Further features comprised systolic, diastolic, and mean flow velocity (MCAv) as well as the difference between systolic and diastolic flow velocity (flow velocity pulse height) and their variation and trends over the same intervals as described for model \#1. Also included were the cerebral autoregulatory computed gain and phase expressed as the transfer function between $\mathrm{P}_{\text {mean }}$ and MCAv over a 3-min moving window between $\mathrm{BP}$ and MCAv [33]. The low frequency band $(0.06-0.15 \mathrm{~Hz})$ where covariation in both signals was significant (coherence of at least 0.5) was averaged to get respective gain and phase. Model \# 5 will further be referred to as flow velocity curve dynamics model (FV curve dynamics). Model 6 and 7 had a single FV derived feature addition. Either the MCAv or flow velocity pulse height were appended to models \#6 and \#7, respectively.

\subsubsection{Further models (models \#8 and \#9)}

Two separate models were created to check model performance without newly introduced features. A model with the basic hemodynamic output from the Nexfin device $\left(\mathrm{P}_{\text {sys }}, \mathrm{P}_{\text {dia }}, \mathrm{P}_{\text {mean }}\right.$, $\mathrm{P}_{\text {pulse }}$, IBI, HR, SV, CO, TPR, and LVET, model \#8) was created to evaluate their additional value compared to $\mathrm{BP}$ and $\mathrm{HR}$. A model comprising of mere $\mathrm{BP}\left(\mathrm{P}_{\text {sys }}, \mathrm{P}_{\text {dia }}\right.$, and $\left.\mathrm{P}_{\text {mean }}\right)$ and $\mathrm{HR}$ was introduced as a basic model (\#9). The number of features in each model is summarized in Table 6.1.

Parameters were transposed into a feature matrix, normalized with respect to values during baseline and scaled so that all features ranged between o and 1. Alongside, a corresponding label vector that contained the appointed class per subject of each feature row was appended.

\section{Training and testing process}

Integral data sets of subjects were included in the modeling algorithm in order to prevent contaminating data from subjects in both training and testing set. Training data consisted of data from a subselection of 30 randomly chosen subjects which changed each iteration. The resulting model was then tested on a single subject who was not part of the training set. This process was repeated for all 42 subjects. The subset of 30 subjects was chosen to reduce total training time. 


\begin{tabular}{|c|c|}
\hline Model names and numbers & Amount of features (on top of base) \\
\hline \#1 BP curve dynamics & $\begin{array}{l}\text { 65: Basic hemodynamics (10 features), curve dynamics } \\
\text { (15 features) and trends and varations ( } 40 \text { features). }\end{array}$ \\
\hline$\# 2 \mathrm{ETCO}_{2}$ & $66\left(\right.$ model $\left.\# 1+\mathrm{ETCO}_{2}\right)$ \\
\hline \#3 TI & $66(\operatorname{model} \# 1+\mathrm{TI})$ \\
\hline \#4 NIRS & 70 (model\#1 +oxygenation parameters ( 5 features $)$ ) \\
\hline \# 5 TCD curve dynamics & $\begin{array}{l}125 \text { (model } \# 1+60 \text { : TCD trends and variation, cerebral } \\
\text { autoregulation) }\end{array}$ \\
\hline \#6 Mean MCAv & 66 (model\#1 +mean TCD MCAv) \\
\hline \#7 MCAv Pulse height & 66 (model\#1 +TCD pulse height) \\
\hline \#8 Volumetric & 10: Basic hemodynamics (10 features) \\
\hline \#9 HR and BP & 4: systolic, diastolic, mean pressures and heart rate. \\
\hline
\end{tabular}

Table 6.1: Model description, numbering and feature count. Models 2 through 7 contain the features from model \#1 with included device specific features. Models 8 and 9 are smaller models, containing features that are currently clinically used and/or available.

\section{Model selection}

Classification success was defined as to what extent a model correctly classifies individual samples. Each successive feature addition returned a unique classification outcome that inor decreased model performance. Each model estimated the probability of a new sample belonging to each of the three classes. Since the classes were defined arbitrarily it is unlikely that the trained models describe a relevant physiological paradigm. To select the best model, and thus its corresponding features, three methods were used to quantify model performance.

1. Actual model sensitivity and specificity.

Sensitivity and specificity per class were the numbers as classified by the trained models without taking into account additional detail of probability estimates of each class. Sensitivity and specificity were calculated on a 1-vs.-all manner.

2. Individual model error.

Model error was expressed as the difference between the predefined classes and the moving average of the prediction of each model.

3. Specificity and sensitivity by accounting for probability estimates.

Next to each model classifying every individual sample, all models return a probability for the sample belonging to each respective class. In method 1 the class with the highest probability is selected as the prediction of the model for that sample. To account for probability estimates we took the ratio of the probability of a sample belonging to class 2 over its probability belonging to class 0 . The logarithm of this (odds) ratio was taken and lower and upper cutoff values for this ratio were determined by using stepwise incremental thresholds to distinguish between classes 0,1 , and 2. The cutoffs were defined as optimal when the sum of both sensitivity and specificity was maximal. 


\subsection{Results}

The results of the search for the optimal $\mathrm{C}$ and gamma values per model are given in Table 6.2. These optimal models were chosen to compute both sensitivity and specificity (Table 6.3), the model errors (Table 6.4) and to detect optimal cutoffs for the probability estimate analysis (Table 6.5).

\subsubsection{Actual model sensitivity and specificity}

Regarding classes 1 and 2, the combination with highest sensitivity and specificity was found for the model comprising volumetric features (\#8) (class 1: sensitivity: 0.73; specificity: 0.98; class 2: sensitivity: 0.56; specificity: 0.96) (Table 6.3). Adding variation, trends and BP curve dynamics (model \#1, Figure 6.2) did not improve the performance of the model for classes 1 (sensitivity 0.63; specificity 0.98) and 2 (sensitivity 0.56; specificity 0.95). Sequentially adding features of $\mathrm{ETCO}_{2}$, TI, or from NIRS or TCD devices also did not improve classifying actual model sensitivity. Specificity was maintained.

\subsubsection{Individual model error}

The FV curve dynamics model (\#5) had the lowest error for all three classes combined (Table 6.4). The median error of the BP curve dynamics (\#1) vs. FV curve dynamics model (\#5) was greater for class 2. The largest fraction of subjects (12/42) benefited from the FV curve dynamics model (\#5) since it had the lowest overall error. Models with either mean MCAv (model \# 6) or pulse height of MCAv (model \#7) accounted for another 8/42 subjects. The BP curve dynamics model (\#1) had the lowest error for 10/42 subjects. Models including $\mathrm{ETCO}_{2}$ (\#2) or NIRS (\#4) both performed best 5/42 times. The TI model (\#3) came in last as the best model in 2 out of 42 subjects.

\begin{tabular}{|lll|}
\hline \multicolumn{1}{|c}{ Feature Set } & C & \multicolumn{1}{c|}{ Gamma } \\
\hline \#1 BP curve dynamics & 0.13895 & 0.002683 \\
\hline \#2 BP curve dynamics \& ETCO & & 0.051795 \\
\hline \#3 BP curve dynamics \&TI & 0.13895 & 0.051795 \\
\hline \#4 BP curve dynamics \&NIRS & 0.13895 & 0.007197 \\
\hline \#5 BP curve dynamics \&TCD & 0.517947 & 0.019307 \\
\hline \#6 BP curve dynamics \& MCAv mean & 100 & 0.13895 \\
\hline \#7 BP curve dynamics \& MCAv pulse height & 0.037276 & 0.001 \\
\hline \#8 Volumetric & 7.196857 & 0.001 \\
\hline \#9 HR and BP & 0.0100 & 0.0027
\end{tabular}

Table 6.2: Optimal model configuration. Optimal results following the 64 -fold optimization steps for different incremental values for regularization parameter C (misclassification penalty) and gamma (deviation of the radial basis Kernel) for each feature set. 


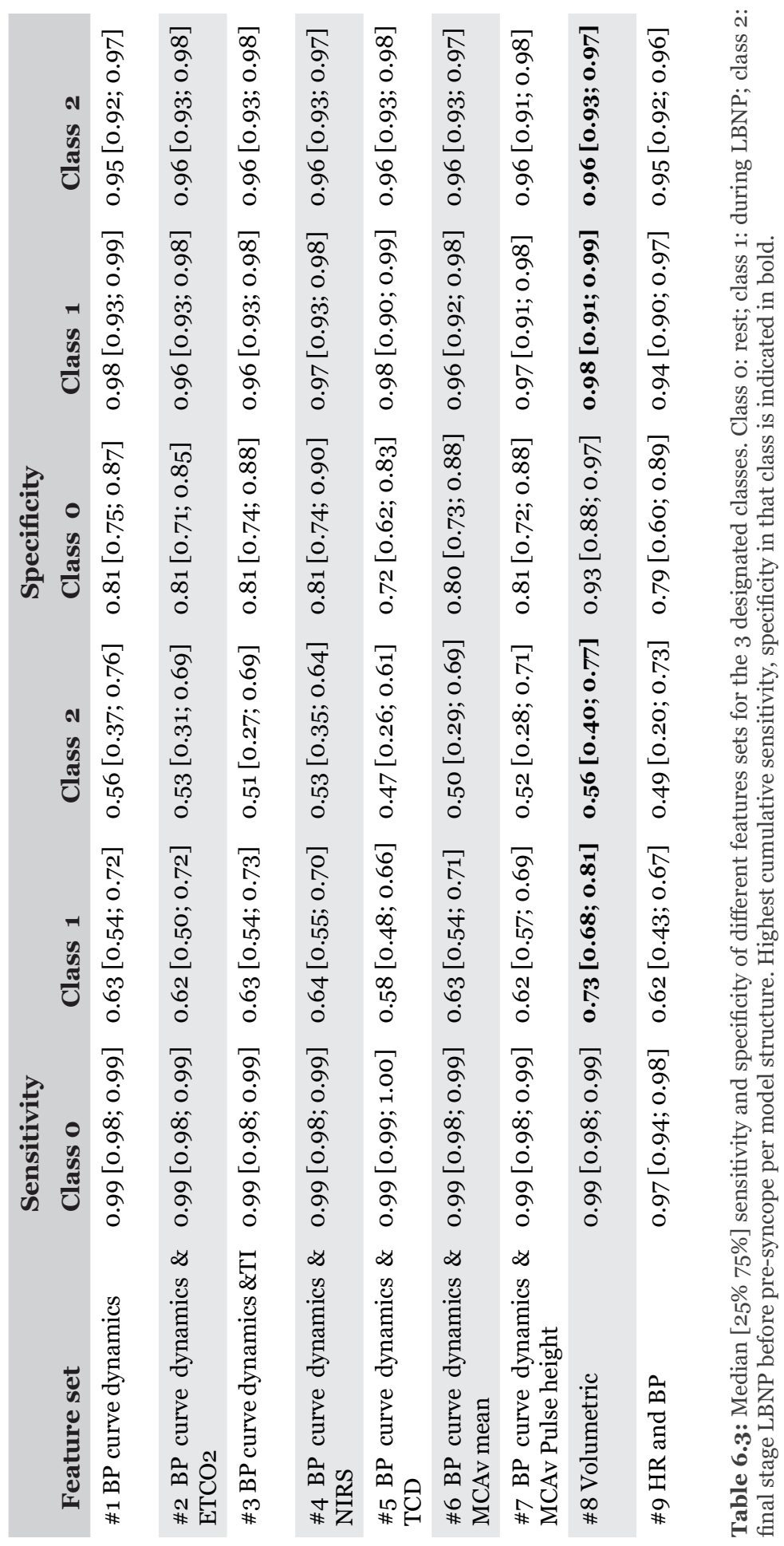




\begin{tabular}{|lllll|}
\hline Class/Model & Class o & Class 1 & Class 2 & $\begin{array}{c}\text { Total } \\
\text { error }\end{array}$ \\
\hline \#1 BP curve dynamics & 0.11 & $\mathbf{0 . 0 6}$ & 0.82 & 1 \\
\hline \#2 ETCO & & 0.12 & 0.65 & 0.89 \\
\hline \#3 TI & 0.13 & 0.11 & 0.67 & 0.89 \\
\hline \#4 NIRS & 0.11 & 0.11 & 0.74 & 0.95 \\
\hline \#5 FV curve dynamics & 0.1 & $\mathbf{0 . 0 6}$ & $\mathbf{0 . 5 3}$ & $\mathbf{0 . 7 8}$ \\
\hline \#6 MCAv mean & 0.11 & 0.1 & 0.7 & 0.91 \\
\hline \#7 MCAv P & 0.11 & 0.11 & 0.81 & 1.03 \\
\hline \#8 Volumetric & $\mathbf{0 . 0 3}$ & 0.07 & 0.71 & 0.82 \\
\hline \#9 HR and BP & 0.12 & 0.16 & 0.81 & 1.09 \\
\hline
\end{tabular}

Table 6.4: Median mean squared errors per model expressed as difference between moving averaged prediction and the predefined class line (Figure 6.3). Lowest error per class indicated in bold.

\subsubsection{Specificity and sensitivity by accounting for probability estimates}

In general, all models had similar sensitivity for baseline (class o) (range 0.89-0.95) and specificity (0.90-0.96) (Table 6.5). Regarding class 1, the best combination of sensitivity and specificity was found for the model that contained the FV derived pulse height (model \#7). The highest combination for class 2 was found for the model with the volumetric features (model \#8). This model also had the highest combination for both class 1 and class 2 together. An overview of all classification samples can be found in the confusion matrices [34] (Supplementary material 6.7.1) for both the actual models and after accounting for probability estimates. In general it can be seen that the models encounter most difficulty in the distinction between class 1 and class 2 while the distinction between class 0 and either class 1 or 2 is clearer.

\subsection{Discussion}

The new findings of the present study are, first, that distinguishing between normovolemia and considerable central hypovolemia in healthy young adults requires information from volumetric hemodynamic features beyond BP and HR, such as IBI, SV, CO, LVET, and TPR. Second, the cerebral blood flow velocity parameters reduced model error, possibly due to the creation of a more easily separable solution.

Features derived from the BP curve, $\mathrm{ETCO}_{2}$, TI, and from cerebral blood flow velocity and brain cortical oxygenation did not improve classification in terms of sensitivity to detect advanced class 2 hypovolemia. In contrast, cerebral blood flow velocity models (\#5-7) outperformed the other models in terms of absolute error from the predefined (artificially created) classes. Models 2-4 (ETCO ${ }_{2}$, central blood volume (TI), and cerebral cortical oxygenation) contributed to such an extent that they were the best discriminative model for fewer subjects and therefore in general seem less sensitive to the detection of CBV depletion. 


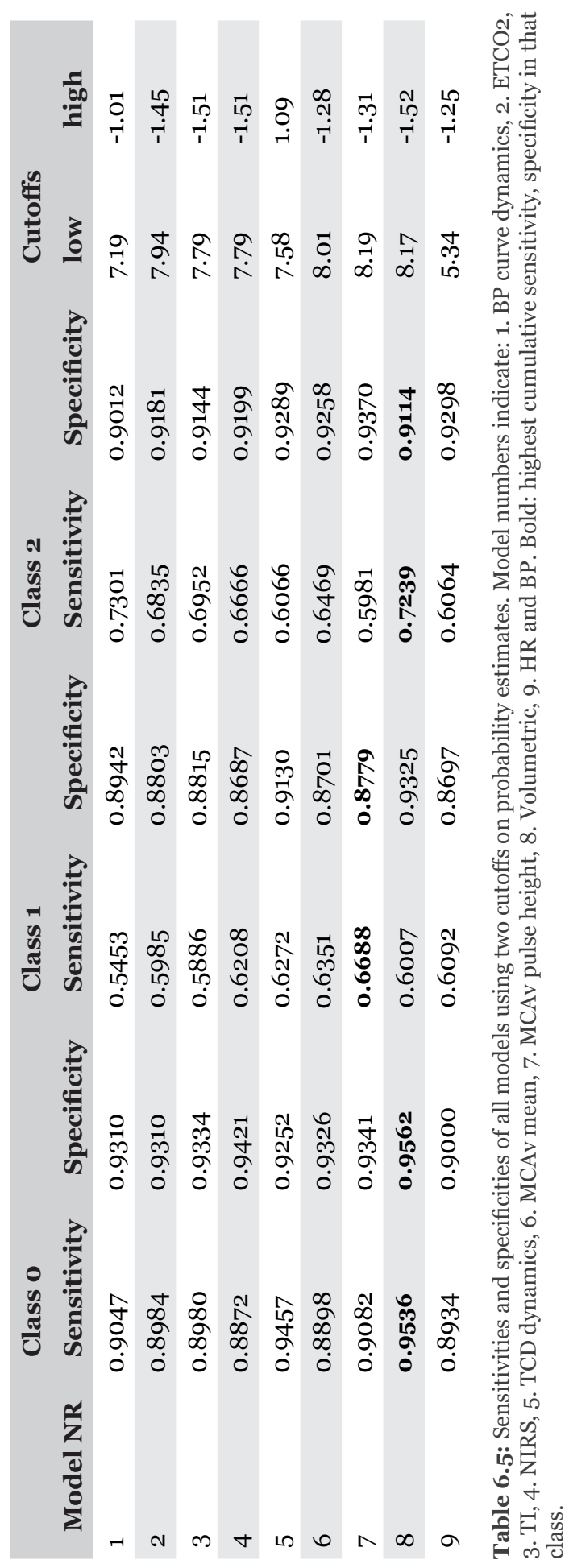



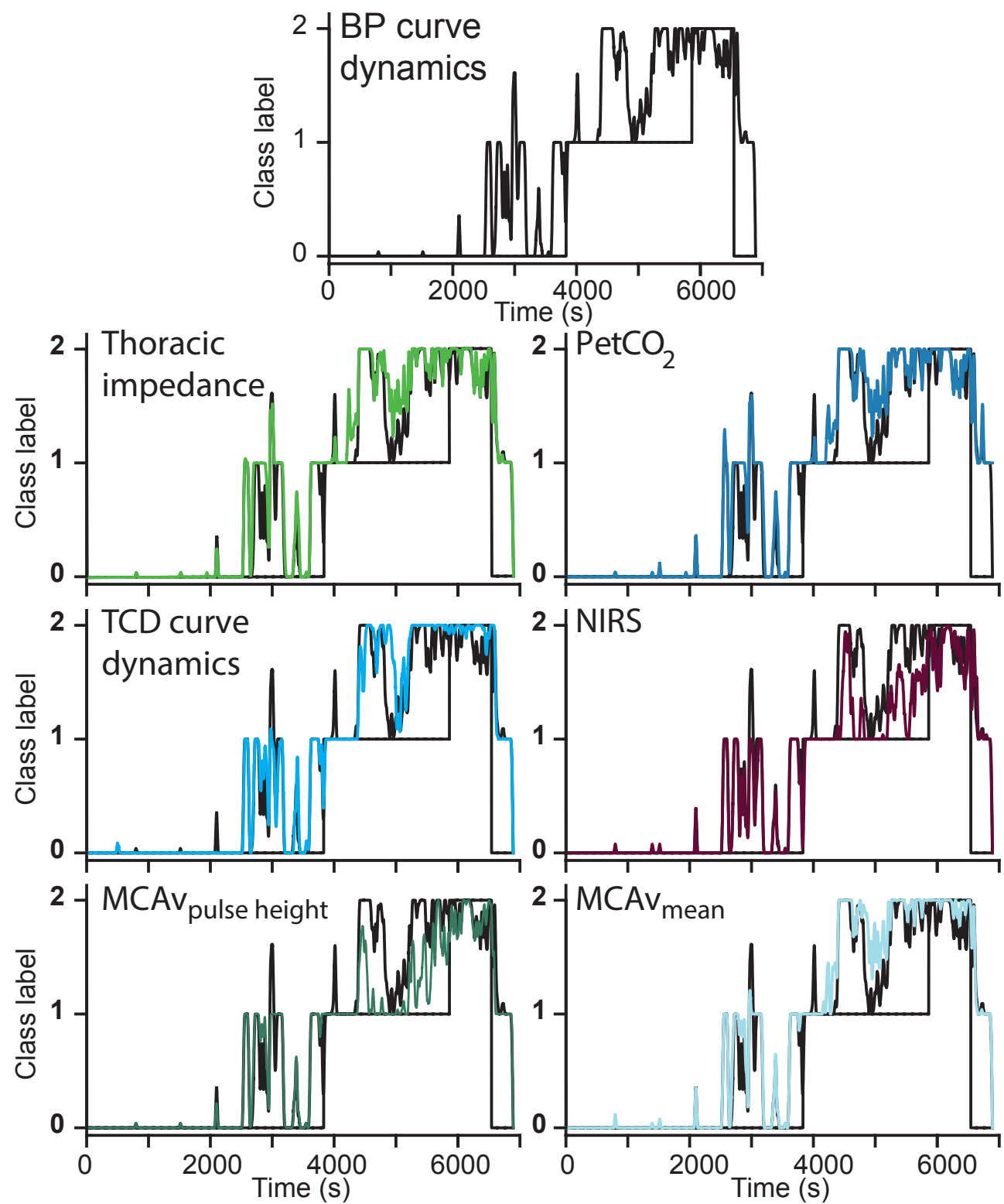

Figure 6.3: Output of six models compared to BP curve dynamics model (\#1, top) in a single subject. Each subsequent graph shows the modulation of the addition of the annotated feature(s). In this subject the model for MCAv pulse height (bottom left) had the lowest error. Note that all model outputs increase with increasing duration of lower body negative pressure. 
In machine learning or datamining approaches large datasets are investigated to determine whether these features together result in a better solution to the problem at hand. A mechanistic approach may not find such a solution in a multidimensional space. The underlying physiological mechanisms can ideally be described by such a mechanistic approach so that it can explain the wide variety of pathophysiology as is seen in different patients. Unfortunately, this is not easily achieved and assumptions would have to be made for many parameters, as they cannot be measured in real time (or at all) resulting in a model that is not very useful for individual cases. Due to the large natural variation between subjects, some individuals increase peripheral resistance to maintain adequate blood pressure, whereas others increase heart rate at onset of LBNP, yet another group responds in a mixed fashion. We do not think these subtleties can be grasped by a mechanistic approach, unless the responses of a patient would be assessed beforehand which is not feasible in clinical practice.

It is possible that a unique set of features exists from different devices that gives an even better solution. To assess this possibility would require a feature selection process which is cumbersome for this amount of models. We considered that these devices are either connected as monitors to patients or not. If so, they return a fixed array of features which was included in the models here. This study therefore aimed to describe which monitors deliver the most sensitive features and should therefore be connected as a monitor for detecting changing CBV.

\subsubsection{Limitations}

By design the subjects were healthy individuals exposed to simulated bleeding which restrains us from extrapolating the data to elderly subjects, considering that with healthy aging brain perfusion becomes increasingly dependent on cardiac output [35].

The current models require that its features are normalized to a reference baseline condition. This will be required as well for future use of the models. Future studies should therefore be directed at finding similar model accuracy without baseline normalization. We recognize that eliminating normalization will increase intersubject scatter, inevitably reducing classification performance.

We consider the possibility that adding a considerable number of features introduced the phenomenon known as overfitting. This would imply that the model is being too specifically trained on training data and may not function equally well on new data. Since the SVM method is a regularization model, the introduction of large amounts of features does not necessarily have to lead to worse performance due to overfitting. However, we selected optimal gamma and $\mathrm{C}$ on the held-out data, which could have led to a form of overfitting, but due to the newly random selection of 30 subjects in the testing step as well, this is expected to be marginal.

Classes were not distributed homogenously. Especially during training this could have had a significant effect on the outcome as the algorithm could have had relatively more examples of what is considered class 2 with respect to the other classes. 
Since the training was performed on a subset of subject data, the reported numbers for sensitivity and specificity are not absolute and will be different if the analysis is repeated. In healthy subjects, variation in cardiovascular responses to sympathetic stimulation evoked by submaximal LBNP is considerable [36]. Differences in resting HR between subjects suggest individually programmed reflex strategies of autonomic blood pressure control which may contribute to the hitherto unpredictable variance observed in cardiovascular reflex responses to central hypovolemia [36]. Due to this large natural variation in subject responses we considered that by using a random subset the models are not focused on a fixed set but will vary with each iteration. Also since not everyone experiences symptoms of pre-syncope in the exact same way there may be a bias toward the point that was defined as pre-syncope here. By using a random subset of individuals the models were never trained on the full set of this bias but included different subjects each training iteration.

\subsubsection{Classification and tracking}

The fact that feature sets from cerebral oxygenation, central blood volume, or cerebral blood flow velocity data do not qualify beats better than the volumetric features seems to suggest that their capability to predict pre-syncope may be low or at least not better than HR and BP combined with LVET, CO, TPR, and SV. However, the probability estimation of class 2 shows a notable increase indicating that in the large majority of subjects the developed models all recognized the process of moving from baseline, to CBV depleted, to pre-syncope.

One explanation for the limited difference in performance between models \#1 and \#8 may be that the Nexfin built-in algorithms in itself include a BP wave shape analysis (pulse contour).

Any attempt to produce a complete clinical classification of hemorrhagic shock for the individual patient can be only provisional due to the complex interrelations in physiological adaptive responses [5,14,37]. Similarly, between healthy subjects the variation in cardiovascular responses to sympathetic stimulation evoked by bleeding is considerable. Distinct cardiovascular response patterns of preferential autonomic blood pressure control appear consistent over time within one subject but with considerable inter-individual variance in tolerance to hypovolemia $[36,38,39]$. This explains the difference in time until pre-syncope and thus differences in the number of samples between subjects available to the models [40]. The models are nevertheless requested to assign one of the three classes to each individual subject through the whole trajectory from normo- to hypovolemia. Also, the large number of samples available for class o compared to class 1 and 2 creates an unequal distribution of samples between the three classes. This also explains the overall high specificity, since classification of a sample not belonging to the investigated class could mean either of two remaining classes.

The translation from model output to underlying physiological events is by no means straightforward. Defining the classes from normo- to hypovolemia served merely to create an artificial distinction between the ongoing circulatory adaptive responses to progressive central hypovolemia. As a consequence, the underlying physiological adaptive responses may not fit into the predefined classes and reported sensitivity does neither reflect direct classification 
of physiology. However, the actual sensitivity/specificity is amenable for improvement by using the certitude of the model by introducing a cut-off analysis on the probability estimates as proposed in order to quantify model performance. This better approaches a classification on a physiological response as changing probabilities of the classes could hint at progression toward cardiovascular instability respectively a return to normovolemia that can be tracked over time.

Ideally, model performance is described by the individual (moving averaged) prediction line as they tend to increase during progressive hypovolemia (Figure 6.3), as a visual manifestation of the increasing probability of impending circulatory collapse since it immediately visualizes into what direction the patient's hemodynamic condition is headed. We attempted to overcome the fact that this measure is difficult to express as a numeric error by implementing three different ways of model performance quantification. This probability estimate analysis increased model sensitivity and specificity by taking into account the complexity of the output of the model in the relative large variation of subject responses to hypovolemia.

\subsection{Conclusion}

Classification of heart beats belonging to either class o or class 1 and 2 is straightforward, and appeared linearly separable using only a few features. This may be due to the fact that this protocol was executed in a controlled setting and due to the fact that the data was normalized to a baseline value. Detecting whether a particular beat should be classified to either the class 1 or class 2 state of being hypovolemic is more challenging, hence the use of a nonlinear Gaussian kernel. Due to the large inter-individual variance and artificial nature of class creation, the data show a considerable overlap for the currently presented features, which hindered us into constructing models with a higher sensitivity. Rather, the moving average during the classification process in itself has the potential to function as a real-time visualization of progress toward hypovolemia induced cardiovascular instability. 


\subsection{References}

1. Grant RT, Reeve EB. Clinical Observations on Air-raid Casualties. $\mathrm{Br}$ Med J. 1941;2(4208):293-7.

2. Secher NH, van Lieshout JJ. Hypovolemic Shock. In: Clinical Fluid Therapy in the Perioperative Setting. 2nd ed. Cambridge: Cambridge University Press; 2016. p. 222-31.

3. Secher NH, Van Lieshout JJ. Normovolaemia defined by central blood volume and venous oxygen saturation. Clin Exp Pharmacol Physiol. 2005;32(11):901-10.

4. Vincent J-L, De Backer D. Circulatory Shock. N Engl J Med. 2013;369(18):1726-1734

5. Perner A, De Backer D. Understanding hypovolaemia. Intensive Care Med. 2014;40(4):613-5.

6. Zöllei É, Bertalan V, Németh A, Csábi P, László I, Kaszaki J, et al. Non-invasive detection of hypovolemia or fluid responsiveness in spontaneously breathing subjects. BMC Anesthesiol. 2013;13(1):40.

7. Simon J, Farkas T, Gingl Z, Csillik A, Korsós A, Rudas L, et al. Noninvasive continuous arterial pressure measurements in the assessment of acute, severe central hypovolemia. Acta Physiol Hung. 2015;102(1):43-50.

8. Marik PE, Monnet X, Teboul J-L. Hemodynamic parameters to guide fluid therapy. Ann Intensive Care. 2011;1(1):1.

9. Bronzwaer A-SGT, Ouweneel DM, Stok WJ, Westerhof BE, van Lieshout JJ. Arterial Pressure Variation as a Biomarker of Preload Dependency in Spontaneously Breathing Subjects - A Proof of Principle. PLoS One. 2015;10(9):e0137364.

10. Harms MPM, Secher NH, van Lieshout JJ. Monitoring of goal-directed fluid challenge. Crit Care Med. 2007;35(2):673-674.

11. Truijen J, Bundgaard-Nielsen M, van Lieshout JJ. A definition of normovolaemia and consequences for cardiovascular control during orthostatic and environmental stress. Eur J Appl Physiol. 2010;109(2):141-57.

12. Bundgaard-Nielsen M, Secher NH, Kehlet H. "Liberal" vs. "restrictive" perioperative fluid therapy--a critical assessment of the evidence. Acta Anaesthesiol Scand. 2009;53(7):843-51.

13. Harms MPM, van Lieshout JJ, Jenstrup M, Pott F, Secher NH. Postural effects on cardiac output and mixed venous oxygen saturation in humans. Exp Physiol. 2003 ;88(5):611-6.

14. Michard F, Teboul J-L. Predicting Fluid Responsiveness in ICU Patients. Chest. 2002;121(6):2000-8.

15. Convertino VA. Blood pressure measurement for accurate assessment of patient status in emergency medical settings. Aviat Space Environ Med. 2012;83(6):614-9.

16. Secher NH. Eat, drink and be merry - and protect the brain. Exp Physiol. 2015;100(9):991991.

17. Cannesson M. Non-invasive guidance of fluid therapy. In: Clinical Fluid Therapy in the Perioperative Setting. 2nd ed. Cambridge: Cambridge University Press; 2016. p. 120-7.

18. Deo RC. Machine Learning in Medicine. Circulation. 2015;132(20):1920-30.

19. Convertino VA, Wirt MD, Glenn JF, Lein BC. The Compensatory Reserve For Early and Accurate Prediction Of Hemodynamic Compromise: A Review of the Underlying Physiology. Shock. 2016;45(6):580-90.

20. Convertino VA, Sawka MN. Wearable technology for compensatory reserve to sense hypovolemia. J Appl Physiol. 2018;124(2):442-51. 
21. Moulton SL, Mulligan J, Grudic GZ, Convertino V a. Running on empty? The compensatory reserve index. J Trauma Acute Care Surg. 2013;75(6):1053-9.

22. Bennis FC, van der Ster BJ, van Lieshout JJ, Andriessen P, Delhaas T. A machinelearning based analysis for the recognition of progressive central hypovolemia. Physiol Meas. 2017;38(9):1791-801.

23. Goswami N, Grasser E, Roessler A, Sschneditz D, Hingofer-Szalkay H. The cardiovascular response to lower body negative pressure in humans depends on seal location. Physiol Res. 2009;58(3):311-8.

24. Bronzwaer A-SGT, Verbree J, Stok WJ, Daemen MJAP, van Buchem MA, van Osch MJP, et al. The cerebrovascular response to lower-body negative pressure vs. head-up tilt. J Appl Physiol. 2017;122(4):877-83.

25. Krantz T, Cai Y, Lauritsen T, Warberg J, Secher NH. Accurate monitoring of blood loss: thoracic electrical impedance during hemorrhage in the pig. Acta Anaesthesiol Scand. 2000;44(5):598-604.

26. van Lieshout JJ, Harms MPM, Pott F, Jenstrup M, Secher NH. Stroke volume of the heart and thoracic fluid content during head-up and head-down tilt in humans. Acta Anaesthesiol Scand. 2005;49(9):1287-92.

27. Rasmussen P, Dawson EA, Nybo L, van Lieshout JJ, Secher NH, Gjedde A. CapillaryOxygenation-Level-Dependent Near-Infrared Spectrometry in Frontal Lobe of Humans. J Cereb Blood Flow Metab. 2007;27(5):1082-93.

28. Gersten A. Probing Brain Oxygenation with Near Infrared Spectroscopy (NIRS) - The Role of Carbon Dioxide and Blood Pressure. In: Infrared Spectroscopy - Anharmonicity of Biomolecules, Crosslinking of Biopolymers, Food Quality and Medical Applications. InTech; 2015. p. 185-7.

29. Claassen JAHR, Colier WNJM, Jansen RWMM. Reproducibility of cerebral blood volume measurements by near infrared spectroscopy in 16 healthy elderly subjects. Physiol Meas. 2006;27(3):255-64.

30. Chang C-C, Lin C-J. LIBSVM: A Library for Support Vector Machines. ACM Trans Intell Syst Technol. 2011;2(3):21-7.

31. Bishop CM. Pattern Recognition and Machine Learning. Cambridge: Springer Science and Business Media LLC; 2006.

32. Chih-Wei Hsu, Chih-Jen Lin. A comparison of methods for multiclass support vector machines. IEEE Trans Neural Networks. 2002;13(2):415-25.

33. Zhang R, Zuckerman JH, Giller CA, Levine BD. Transfer function analysis of dynamic cerebral autoregulation in humans. Am J Physiol Circ Physiol. 1998;274(1):H233-41.

34. Stehman S V. Selecting and interpreting measures of thematic classification accuracy. Remote Sens Environ. 1997;62(1):77-89.

35. Bronzwaer A-SGT, Verbree J, Stok WJ, Daemen MJAP, van Buchem MA, van Osch MJP, et al. Aging modifies the effect of cardiac output on middle cerebral artery blood flow velocity. Physiol Rep. 2017;5(17).

36. Bronzwaer A-SGT, Verbree J, Stok WJ, van Buchem MA, Daemen MJAP, van Osch MJP, et al. Cardiovascular Response Patterns to Sympathetic Stimulation by Central Hypovolemia. Front Physiol. 2016;7:235.

37. McMichael J. Clinical aspects of shock. J Am Med Assoc. 1944;124(5):275. 
38. Convertino VA, Rickards CA, Ryan KL. Autonomic mechanisms associated with heart rate and vasoconstrictor reserves. Clin Auton Res Off J Clin Auton Res Soc. 2012;22(3):123-30.

39. Ryan KL, Rickards CA, Hinojosa-Laborde C, Cooke WH, Convertino VA. Sympathetic Responses to Central Hypovolemia: New Insights from Microneurographic Recordings. Front Physiol. 2012;3:110.

40. Jellema WT, Imholz BPM, Van Goudoever J, Wesseling KH, Van Lieshout JJ. Finger Arterial versus Intrabrachial Pressure and Continuous Cardiac output during Head-up Tilt Testing in Healthy Subjects. Clin Sci. 1996;91(2):193-200. 


\subsection{Supplementary materials}

\begin{tabular}{|c|c|c|}
\hline Feature name & $\begin{array}{l}\text { Feature description and } \\
\text { explanation of Figure } 6.2\end{array}$ & Equation \\
\hline A & Position of A relative to systole & - \\
\hline B & Systole & - \\
\hline $\mathrm{C}$ & Dicrotic notch; relative to systole & - \\
\hline $\mathrm{D}$ & Position of $\mathrm{D}$ relative to systole & - \\
\hline $\mathrm{E}$ & $\begin{array}{l}\text { Position of E relative to systole (red dot in } \\
\text { Figure 6.2) }\end{array}$ & - \\
\hline $\mathrm{a}$ & Time point of A relative to e & - \\
\hline $\mathrm{b}$ & Time point of $\mathrm{B}$ relative to e & - \\
\hline c & Time point of $\mathrm{C}$ relative to e & - \\
\hline $\mathrm{d}$ & Time point of $\mathrm{D}$ relative to e & - \\
\hline tangAB & $\begin{array}{l}\text { Slope between points A and B (dashed line } \\
\text { I in Figure 6.2) }\end{array}$ & $\frac{B-A}{b-a}$ \\
\hline tangBC & $\begin{array}{l}\text { Slope between points B and C (dashed line } \\
\text { II in Figure 6.2) }\end{array}$ & $\frac{C-B}{c-b}$ \\
\hline tangCD & $\begin{array}{l}\text { Slope between points } \mathrm{C} \text { and } \mathrm{D} \text { (dashed line } \\
\text { III in Figure 6.2) }\end{array}$ & $\frac{D-C}{d-c}$ \\
\hline tangDE & $\begin{array}{l}\text { Slope between points D and E (dashed line } \\
\text { IV in Figure 6.2) }\end{array}$ & $\frac{E-D}{e-d}$ \\
\hline ratBC & Ratio between points B and C & $\frac{B}{C}$ \\
\hline ratCD & Ratio between points B and C & $\frac{C}{D}$ \\
\hline ratBD & Ratio between points B and D & $\frac{D}{B}$ \\
\hline surAB & $\begin{array}{l}\text { Line integral of the BP curve for time } \\
\text { segment a to b (light area in Figure 6.2) }\end{array}$ & $\int_{a}^{b} f(t)$ \\
\hline surAE & $\begin{array}{l}\text { Line integral of the entire BP curve (dark } \\
\text { \& light area in Figure } 6.2 \text { ) }\end{array}$ & $\int_{a}^{e} f(t)$ \\
\hline surCE & $\begin{array}{l}\text { Line integral of the BP curve for time } \\
\text { segment c to e (dark area in Figure 6.2) }\end{array}$ & $\int_{a}^{c} f(t)$ \\
\hline
\end{tabular}

Table S6.1: Continued on next page. 


\begin{tabular}{|c|c|c|}
\hline Feature name & $\begin{array}{l}\text { Feature description and } \\
\text { explanation of Figure } 6.2\end{array}$ & Equation \\
\hline ratSurAB_AE & ratio of surface $A$ to $B$ to $A$ to $E$ & $\frac{\int_{a}^{b} f(t)}{\int_{a}^{e} f(t)}$ \\
\hline ratSurCE_AE & ratio of surface $\mathrm{C}$ to $\mathrm{E}$ to $\mathrm{A}$ to $\mathrm{E}$ & $\frac{\int_{c}^{e} f(t)}{\int_{a}^{e} f(t)}$ \\
\hline ratSurCE_AB & ratio of surface $\mathrm{C}$ to $\mathrm{E}$ to $\mathrm{A}$ to $\mathrm{B}$ & $\frac{\int_{c}^{e} f(t)}{\int_{n}^{b} f(t)}$ \\
\hline DPDTMax & $\begin{array}{l}\text { Max slope of the BP curve over segment } \\
\text { A to B }\end{array}$ & {$\left[\max \left(\frac{\Delta P}{d t}\right)\right]_{a}^{b}$} \\
\hline $\begin{array}{l}\text { Height of dicrotic } \\
\text { notch }\end{array}$ & $\begin{array}{l}\text { Local minimum surrounding LVET } \\
\text { normalized to systolic pressure. }\end{array}$ & - \\
\hline
\end{tabular}

Table S6.1: Continuation from previous page. Blood pressure wave shape is indicated with $\mathrm{F}(\mathrm{t})$. Detected points A through $\mathrm{E}$ and their corresponding times A through $\mathrm{E}$ are not included as features but only serve for explanation purposes. 


\subsubsection{Confusion matrices}

In this appendix the confusion matrix of both steps in error calculation are reported. On the left is the initial accuracy for each model and on the right each model accuracy after computing the optimal cut-off values for the probability estimates. These cut-offs were defined optimal once the distinction between class 1 and class 2 was the highest since this was deemed clinically the most important.

Each cell has two numbers. For the first 3 rows, the absolute numbers indicated the number of samples classified like this. The percentage in that cell indicates the proportion of the sample of this cell compared to the complete data set size it was tested on. Column and row 4 have two percentage numbers: the top one indicating the amount of correct classifications, the bottom number indicating the number of mistakes. Horizontally these indicate the amount of times a prediction is correct. Vertically this indicates the amount of times a target class is classified correctly.

The diagonal cells show the number and percentage of correct classifications.

Model 1. Blood pressure curve dynamics

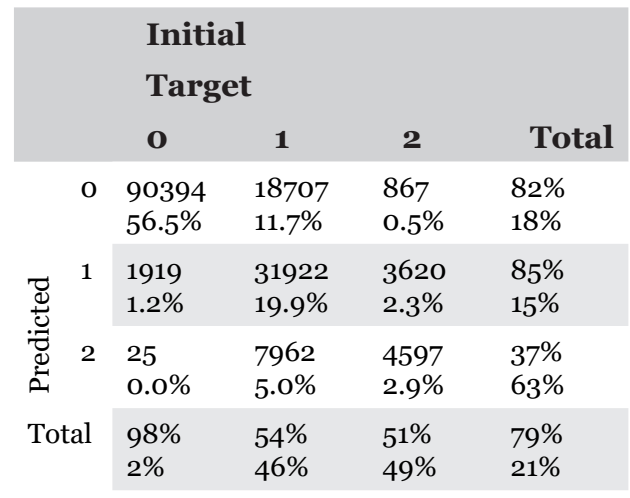

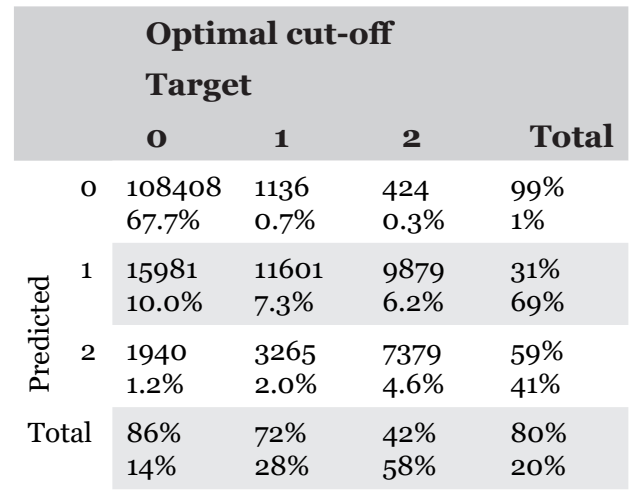


Model 2. $\mathrm{ETCO}_{2}$

\begin{tabular}{|c|c|c|c|c|}
\hline & \multicolumn{2}{|c|}{$\begin{array}{l}\text { Initial } \\
\text { Target }\end{array}$} & \multirow[b]{2}{*}{2} & \multirow[b]{2}{*}{ Total } \\
\hline & o & 1 & & \\
\hline o & $\begin{array}{l}88848 \\
55.5 \%\end{array}$ & $\begin{array}{l}20011 \\
12.5 \%\end{array}$ & $\begin{array}{l}1109 \\
0.7 \%\end{array}$ & $\begin{array}{l}81 \% \\
19 \%\end{array}$ \\
\hline$\sigma^{\circ} 1$ & $\begin{array}{l}1796 \\
1.1 \%\end{array}$ & $\begin{array}{l}29622 \\
18.5 \%\end{array}$ & $\begin{array}{l}6043 \\
3.8 \%\end{array}$ & $\begin{array}{l}79 \% \\
21 \%\end{array}$ \\
\hline 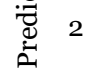 & $\begin{array}{l}28 \\
0.0 \%\end{array}$ & $\begin{array}{l}6737 \\
4.2 \%\end{array}$ & $\begin{array}{l}5819 \\
3.6 \%\end{array}$ & $\begin{array}{l}46 \% \\
54 \%\end{array}$ \\
\hline Total & $\begin{array}{l}98 \% \\
2 \%\end{array}$ & $\begin{array}{l}53 \% \\
47 \%\end{array}$ & $\begin{array}{l}45 \% \\
55 \%\end{array}$ & $\begin{array}{l}78 \% \\
22 \%\end{array}$ \\
\hline
\end{tabular}

\begin{tabular}{|c|c|c|c|c|}
\hline & \multicolumn{3}{|c|}{$\begin{array}{l}\text { Optimal cut-off } \\
\text { Target }\end{array}$} & \multirow[b]{2}{*}{ Total } \\
\hline & $\mathbf{o}$ & 1 & 2 & \\
\hline \multirow{3}{*}{ 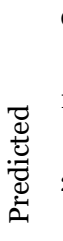 } & $\begin{array}{l}107537 \\
67.2 \%\end{array}$ & $\begin{array}{l}2104 \\
1.3 \%\end{array}$ & $\begin{array}{l}327 \\
0.2 \%\end{array}$ & $\begin{array}{l}98 \% \\
2 \%\end{array}$ \\
\hline & $\begin{array}{l}12443 \\
7.8 \%\end{array}$ & $\begin{array}{l}15690 \\
9.8 \%\end{array}$ & $\begin{array}{l}9328 \\
5.8 \%\end{array}$ & $\begin{array}{l}42 \% \\
58 \%\end{array}$ \\
\hline & $\begin{array}{l}1199 \\
0.7 \%\end{array}$ & $\begin{array}{l}3871 \\
2.4 \%\end{array}$ & $\begin{array}{l}7514 \\
4.7 \%\end{array}$ & $\begin{array}{l}60 \% \\
40 \%\end{array}$ \\
\hline Total & $\begin{array}{l}89 \% \\
11 \%\end{array}$ & $\begin{array}{l}72 \% \\
28 \%\end{array}$ & $\begin{array}{l}44 \% \\
56 \%\end{array}$ & $\begin{array}{l}82 \% \\
18 \%\end{array}$ \\
\hline
\end{tabular}

Model 3. Thoracic impedance

\begin{tabular}{|c|c|c|c|c|}
\hline & \multicolumn{2}{|c|}{$\begin{array}{l}\text { Initial } \\
\text { Target }\end{array}$} & \multirow[b]{2}{*}{2} & \multirow[b]{2}{*}{ Total } \\
\hline & $\mathbf{O}$ & 1 & & \\
\hline \multirow{3}{*}{ 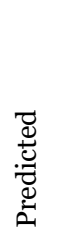 } & $\begin{array}{l}90137 \\
56.3 \%\end{array}$ & $\begin{array}{l}18634 \\
11.6 \%\end{array}$ & $\begin{array}{l}1197 \\
0.7 \%\end{array}$ & $\begin{array}{l}82 \% \\
18 \%\end{array}$ \\
\hline & $\begin{array}{l}1868 \\
1.2 \%\end{array}$ & $\begin{array}{l}29777 \\
18.6 \%\end{array}$ & $\begin{array}{l}5816 \\
3.6 \%\end{array}$ & $\begin{array}{l}79 \% \\
21 \%\end{array}$ \\
\hline & $\begin{array}{l}27 \\
0.0 \%\end{array}$ & $\begin{array}{l}7107 \\
4.4 \%\end{array}$ & $\begin{array}{l}5450 \\
3.4 \%\end{array}$ & $\begin{array}{l}43 \% \\
57 \%\end{array}$ \\
\hline Tota & $\begin{array}{l}98 \% \\
2 \%\end{array}$ & $\begin{array}{l}54 \% \\
46 \%\end{array}$ & $\begin{array}{l}44 \% \\
56 \%\end{array}$ & $\begin{array}{l}78 \% \\
22 \%\end{array}$ \\
\hline
\end{tabular}

\begin{tabular}{|c|c|c|c|c|}
\hline & \multicolumn{3}{|c|}{$\begin{array}{l}\text { Optimal cut-off } \\
\text { Target }\end{array}$} & \multirow[b]{2}{*}{ Total } \\
\hline & $\mathbf{O}$ & $\mathbf{1}$ & 2 & \\
\hline \multirow{3}{*}{ 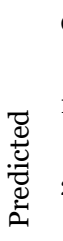 } & $\begin{array}{l}107690 \\
67.3 \%\end{array}$ & $\begin{array}{l}1967 \\
1.2 \%\end{array}$ & $\begin{array}{l}311 \\
0.2 \%\end{array}$ & $\begin{array}{l}98 \% \\
2 \%\end{array}$ \\
\hline & $\begin{array}{l}13246 \\
8.3 \%\end{array}$ & $\begin{array}{l}15329 \\
9.6 \%\end{array}$ & $\begin{array}{l}8886 \\
5.6 \%\end{array}$ & $\begin{array}{l}41 \% \\
59 \%\end{array}$ \\
\hline & $\begin{array}{l}1253 \\
0.8 \%\end{array}$ & $\begin{array}{l}4089 \\
2.6 \%\end{array}$ & $\begin{array}{l}7242 \\
4.5 \%\end{array}$ & $\begin{array}{l}58 \% \\
42 \%\end{array}$ \\
\hline Total & $\begin{array}{l}88 \% \\
12 \%\end{array}$ & $\begin{array}{l}72 \% \\
28 \%\end{array}$ & $\begin{array}{l}44 \% \\
56 \%\end{array}$ & $\begin{array}{l}81 \% \\
19 \%\end{array}$ \\
\hline
\end{tabular}

Model 4. NIRS

\begin{tabular}{|c|c|c|c|c|}
\hline & \multicolumn{2}{|c|}{$\begin{array}{l}\text { Initial } \\
\text { Target }\end{array}$} & \multirow[b]{2}{*}{2} & \multirow[b]{2}{*}{ Total } \\
\hline & $\mathbf{O}$ & 1 & & \\
\hline \multirow{3}{*}{ 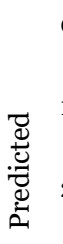 } & $\begin{array}{l}90262 \\
56.4 \%\end{array}$ & $\begin{array}{l}18688 \\
11.7 \%\end{array}$ & $\begin{array}{l}1018 \\
0.6 \%\end{array}$ & $\begin{array}{l}82 \% \\
18 \%\end{array}$ \\
\hline & $\begin{array}{l}1889 \\
1.2 \%\end{array}$ & $\begin{array}{l}31544 \\
19.7 \%\end{array}$ & $\begin{array}{l}4028 \\
2.5 \%\end{array}$ & $\begin{array}{l}84 \% \\
16 \%\end{array}$ \\
\hline & $\begin{array}{l}26 \\
0.0 \%\end{array}$ & $\begin{array}{l}7236 \\
4.5 \%\end{array}$ & $\begin{array}{l}5322 \\
3.3 \%\end{array}$ & $\begin{array}{l}42 \% \\
58 \%\end{array}$ \\
\hline Total & $\begin{array}{l}98 \% \\
2 \%\end{array}$ & $\begin{array}{l}55 \% \\
45 \%\end{array}$ & $\begin{array}{l}51 \% \\
49 \%\end{array}$ & $\begin{array}{l}79 \% \\
21 \%\end{array}$ \\
\hline
\end{tabular}

\begin{tabular}{|c|c|c|c|c|}
\hline & \multicolumn{3}{|c|}{$\begin{array}{l}\text { Optimal cut-off } \\
\text { Target }\end{array}$} & \multirow[b]{2}{*}{ Total } \\
\hline & o & 1 & 2 & \\
\hline \multirow{3}{*}{ 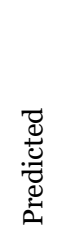 } & $\begin{array}{l}108645 \\
67.9 \%\end{array}$ & $\begin{array}{l}1002 \\
0.6 \%\end{array}$ & $\begin{array}{l}321 \\
0.2 \%\end{array}$ & $\begin{array}{l}99 \% \\
1 \%\end{array}$ \\
\hline & $\begin{array}{l}16705 \\
10.4 \%\end{array}$ & $\begin{array}{l}11812 \\
7.4 \%\end{array}$ & $\begin{array}{l}8944 \\
5.6 \%\end{array}$ & $\begin{array}{l}32 \% \\
68 \%\end{array}$ \\
\hline & $\begin{array}{l}1844 \\
1.2 \%\end{array}$ & $\begin{array}{l}3416 \\
2.1 \%\end{array}$ & $\begin{array}{l}7324 \\
4.6 \%\end{array}$ & $\begin{array}{l}58 \% \\
42 \%\end{array}$ \\
\hline Total & $\begin{array}{l}85 \% \\
15 \%\end{array}$ & $\begin{array}{l}73 \% \\
27 \%\end{array}$ & $\begin{array}{l}44 \% \\
56 \%\end{array}$ & $\begin{array}{l}80 \% \\
20 \%\end{array}$ \\
\hline
\end{tabular}


Model 5. TCD curve dynamics

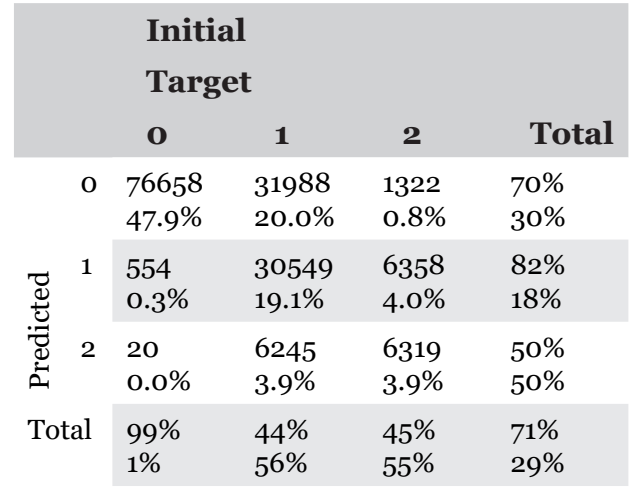

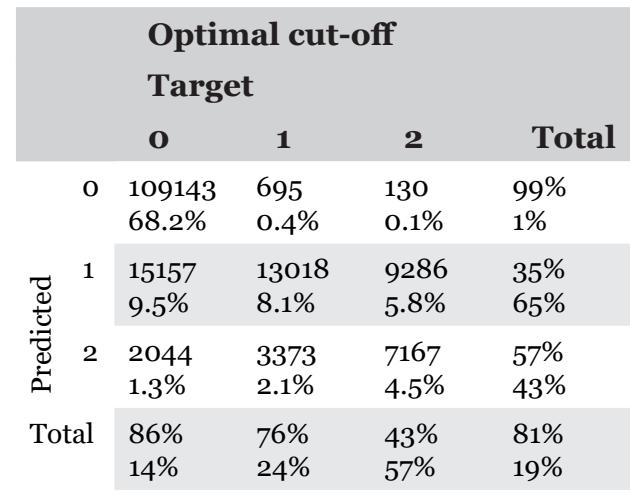

\section{Model 6. Mean MCAv}

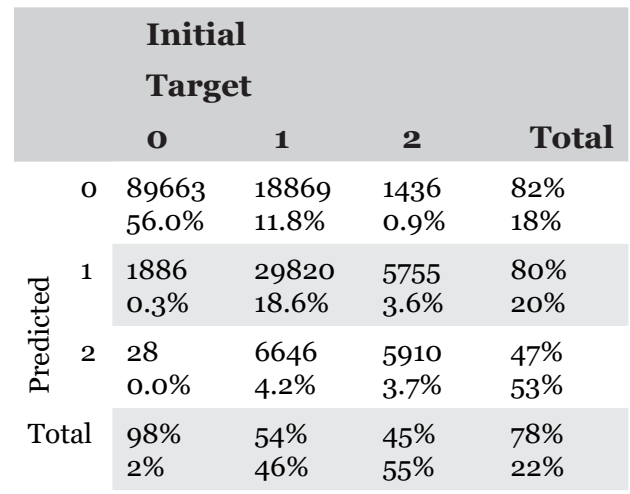

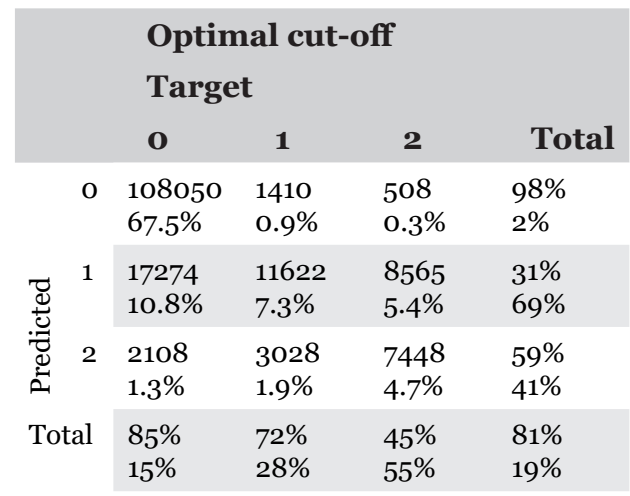

\section{Model 7. MCAv pulse height}

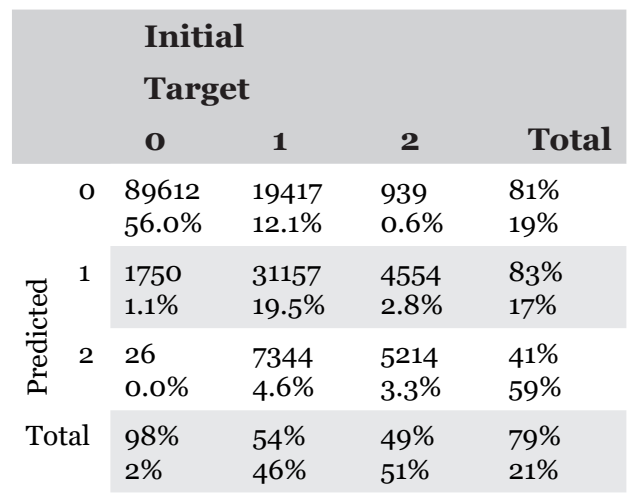

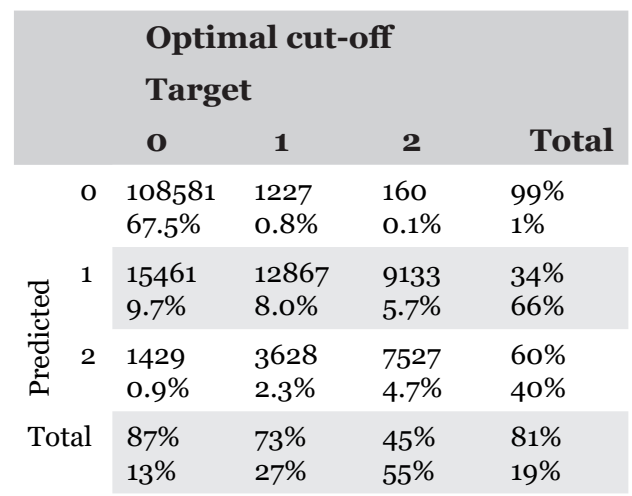


100 | Chapter 6

Model 8. Volumetric

\begin{tabular}{|c|c|c|c|c|c|}
\hline & \multicolumn{2}{|c|}{$\begin{array}{l}\text { Initial } \\
\text { Target }\end{array}$} & \multirow[b]{2}{*}{2} & \multirow[b]{2}{*}{ Total } \\
\hline & & $\mathbf{0}$ & 1 & & \\
\hline \multirow{3}{*}{ 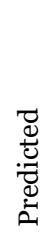 } & O & $\begin{array}{l}103189 \\
64.5 \%\end{array}$ & $\begin{array}{l}6076 \\
3.8 \%\end{array}$ & $\begin{array}{l}703 \\
0.4 \%\end{array}$ & $\begin{array}{l}94 \% \\
6 \%\end{array}$ \\
\hline & 1 & $\begin{array}{l}2090 \\
1.3 \%\end{array}$ & $\begin{array}{l}29189 \\
18.2 \%\end{array}$ & $\begin{array}{l}6182 \\
3.9 \%\end{array}$ & $\begin{array}{l}78 \% \\
22 \%\end{array}$ \\
\hline & 2 & $\begin{array}{l}22 \\
0.0 \%\end{array}$ & $\begin{array}{l}7093 \\
4.4 \%\end{array}$ & $\begin{array}{l}5469 \\
3.4 \%\end{array}$ & $\begin{array}{l}43 \% \\
57 \%\end{array}$ \\
\hline \multicolumn{2}{|c|}{ Total } & $\begin{array}{l}98 \% \\
2 \%\end{array}$ & $\begin{array}{l}69 \% \\
31 \%\end{array}$ & $\begin{array}{l}44 \% \\
56 \%\end{array}$ & $\begin{array}{l}86 \% \\
14 \%\end{array}$ \\
\hline
\end{tabular}

\begin{tabular}{|c|c|c|c|c|}
\hline & \multicolumn{3}{|c|}{$\begin{array}{l}\text { Optimal cut-off } \\
\text { Target }\end{array}$} & \multirow[b]{2}{*}{ Total } \\
\hline & $\mathbf{0}$ & 1 & 2 & \\
\hline o & $\begin{array}{l}108765 \\
68.0 \%\end{array}$ & $\begin{array}{l}944 \\
0.6 \%\end{array}$ & $\begin{array}{l}259 \\
0.2 \%\end{array}$ & $\begin{array}{l}99 \% \\
1 \%\end{array}$ \\
\hline 总 1 & $\begin{array}{l}8880 \\
5.5 \%\end{array}$ & $\begin{array}{l}17139 \\
10.7 \%\end{array}$ & $\begin{array}{l}11442 \\
7.2 \%\end{array}$ & $\begin{array}{l}46 \% \\
54 \%\end{array}$ \\
\hline 总 2 & $\begin{array}{l}361 \\
0.2 \%\end{array}$ & $\begin{array}{l}3697 \\
2.3 \%\end{array}$ & $\begin{array}{l}8526 \\
5 \cdot 3 \%\end{array}$ & $\begin{array}{l}68 \% \\
32 \%\end{array}$ \\
\hline Total & $\begin{array}{l}92 \% \\
8 \%\end{array}$ & $\begin{array}{l}79 \% \\
21 \%\end{array}$ & $\begin{array}{l}42 \% \\
58 \%\end{array}$ & $\begin{array}{l}84 \% \\
16 \%\end{array}$ \\
\hline
\end{tabular}

Model 9. Heart rate and blood pressure

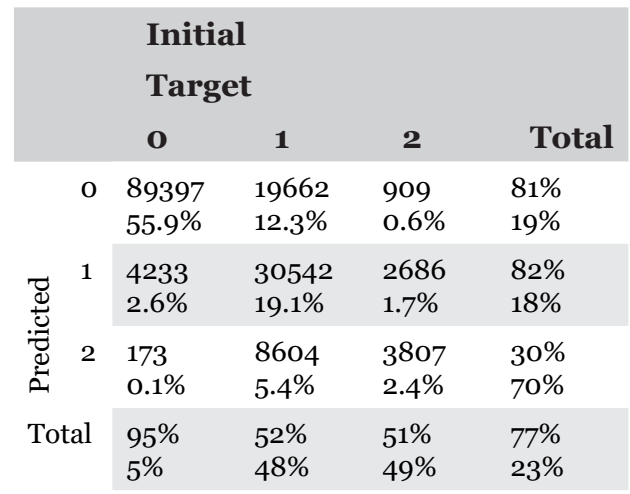

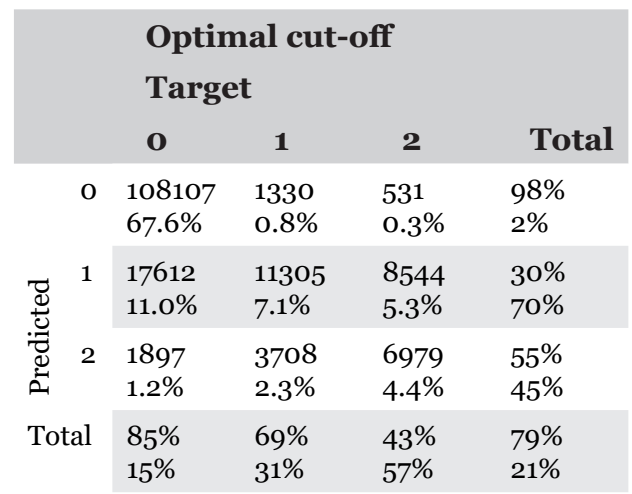






\section{Chapter 7}

General Discussion 
104 | Chapter 7 
The approach for model creation in medicine is not "plug and play". Numerous key aspects must be taken into consideration during model creation. In this thesis we showed the importance of data quality (Chapter 2) and parameter calculation (Chapter 3) and we provided three examples of the implementation of a machine learning model for a clinical problem (Chapter 4-6).

\subsection{Data quality}

Processing and cleaning of data to ensure good data quality is of utmost importance prior to all steps during model creation. In Chapter 2, we investigated the effect of a small synchronization error in the plethysmograph signal on the pulse transit time, i.e. the time difference between the R peak in the ECG and the upslope of the photoplethysmogram. The pulse transit time has been shown to be a possible indicator for changes in the cardiovascular system and corresponding diseases [1-3]. Due to the synchronization error, the plethysmograph waveform was each beat stretched with a very small time period, which was compensated by a compressed beat after a constant time interval. Although this error had no effect on the oxygenation level displayed, it did influence the time difference between the $\mathrm{R}$ peak in the ECG and the upslope of the photoplethysmogram. This resulted in a sawtooth artifact and an additional constant bias in the pulse transit time. These artifacts were large enough to render the thus-derived pulse transit time useless as a parameter for clinical evaluation.

Although studies regarding the pulse transit time do use artifact-free data they often don't report their definition of artifact-free data neither do they check the synchronization between the plethysmogram and the ECG [4,5]. It is worrisome that some clinical models are based on parameters that are not checked for their accuracy, such as the use of the pulse transit time as a parameter to predict hypotensive events without checking for a sawtooth artifact or even mentioning the used plethysmogram sensor [6]. Using erroneous parameters as input in clinical models decreases the accuracy since the accuracy of a model depends on the quality of the data provided $[7,8]$. To prevent the construction of such errors, data quality should be controlled.

Assessment of data quality depends on multiple important things. 1). The measured parameter has to be accurate in amplitude and timing, as shown in the example of the pulse transit time. Furthermore, data should contain as few as possible artifacts. To this end, data should be cleaned of basic measurement errors such as blood pressure artifacts. 2). A parameter should be similar across measurement devices to make a model generalizable to other hospitals with different measurement devices. For instance, automatic blood pressure measurements are known to be vendor-dependent and to differ from manual blood pressure measurements $[9,10]$, indicating that a model based on data obtained with measurement device ' $\mathrm{A}$ ' is not generalizable to data obtained with other devices. Therefore, data should be ideally measured with a similar device or it should be vendor-independent 3). Data should accurately reflect the underlying physiology and incorporate patients with different background and social status. An important issue regarding data quality is algorithmic bias, which tends to create biased results for different groups of patients [11]. Examples are known in which large well-trained models underperform on socioeconomically less fortunate patients or on data from low- 
income countries $[12,13]$. Because models like the CRASH study, which is used to predict the outcome after six months in patients with traumatic brain injury, are based on the standard of care in high-income countries, the accuracy will be less in data obtained from low-income countries as the standard of care is lower. Whilst this is not always avoidable, it has to be taken into account when applying a model to a new patient.

\subsection{Parameter extraction}

Raw clinical patient data is in general first processed into a parameter before it is used in a machine learning model [14]. The extraction of such a parameter is usually based on domain knowledge. Parameter extraction based on domain knowledge ensures that the clinician can relate the changes in the human body to the outcome given by the model $[15,16]$. However, it occurs more and more that raw clinical data is immediately inserted in a complex model [17]. The use of raw data is less time-consuming and may result in a good model performance, though the connection to the underlying physiology is unknown. Furthermore, it is unknown whether the found relationship between input and output is correlated or causally related, which may lead to wrong predictions when generalizing to new data. An example in which the use of raw clinical data resulted in errors in diagnosis is found in the detection of melanoma [18]. Images of a possible melanoma were inserted into a model using the pixels as parameters. However, the images contained a surgical skin marking, which affected the prediction. The model based its decision of the presence of melanoma partly on the marking, which is artificial and undesirable for use in clinical situations. Parameters based on domain knowledge of the clinician, such as shape and color of the possible melanoma, would not use the surgical skin marking as a parameter. Therefore, it is important to extract parameters from the raw clinical data.

In Chapter 3, we developed a parameter that calculates the ratio for the in- and decrease in blood pressure surrounding the systolic peak in a continuous blood pressure waveform. The ratio is built upon physiological grounds, aiding interpretability of this parameter. This ratio could identify the presence of a patent ductus arteriosus in preterm infants with 83.3\% accuracy. Furthermore, this ratio is based on data measured during standard of care, implying the parameter can be calculated without need for additional data collection during admission.

The high accuracy obtained in Chapter 3 using a single parameter demonstrates the importance of taking the underlying physiology into account during parameter construction. The thus created parameter can already independently discriminate groups, like in our study patients with closed and patent ductus arteriosus. The combination of several of such interpretable parameters may result in a model with an even higher accuracy. Furthermore, parameter extraction leads to fewer parameters compared to using raw data, which results to less chance of overfitting and a lower number of samples needed $[16,19]$. Therefore, although it is tempting to directly use raw data in a machine learning model while obtaining good results, parameter extraction is an important step in clinical model creation. 


\subsection{Model creation}

\subsubsection{Machine learning models}

Machine learning models are statistical tools to describe a function between input and output [20]. A machine learning model can combine a large number of parameters (input) to obtain an accurate prediction or diagnosis (output) in clinical situations. A large number of machine learning models exist, some more commonly used than others. We discuss four often-used models: logistic regression, support vector machine, decision trees and neural networks. These models differ in complexity and transparency [21].

A logistic regression model assumes groups can be separated linearly. This kind of model fits a linear relationship in which a single weight is given to each parameter [20,22,23]. Evaluating these weights gives insight in the importance of each parameter in the model. Therefore, it is possible to understand how the model reaches a decision. However, due to the assumption of linearity, complex non-linear relationships between input and output can only be modeled by explicitly introducing them in the parameters [24].

Support vector machines use a so-called kernel to transform input data to a different parameter space [23,25]. A non-linear kernel transforms the data non-linearly. Though this enables the model to create complex non-linear input / output relationships, it becomes harder to follow why the model reaches a certain prediction [26]. A linear kernel enables the evaluation of the importance of each parameter similar to logistic regression but is not able to fit non-linear relationships. Therefore, depending on the kernel used in the support vector machine model, transparency may be high or low.

Decision trees are interpretable, tree-like based models [23,27]. Starting from a single point, the tree branches out in two branches. If a parameter is below a certain threshold, you follow one branch of the tree; otherwise, you follow the other branch of the three. After following the branch, the branch branches again, evaluating a different threshold for a possibly different parameter. An example is found during the assessment whether resuscitation is necessary during basic life support. The decision tree starts with "Does the patient respond? If yes, call for help. If not, does the patient breath? If not, call the emergency department and start reanimation" [28]. Although this example is only the start of a very simple decision tree, it is comparable to how a clinician may view a patient. Therefore, this model clearly indicates how a certain prediction is reached, while it is also able to fit non-linear relationships. However, a decision tree usually shows lower accuracy [23].

Neural networks are models able to fit complex non-linear relationships between input and output [29]. Between the input parameters and the output is at least one layer with a number of nodes in it, though in general the number of layers and the number of nodes is large. All input parameters are connected to each node, where a non-linear algorithm combines these parameters into one value that is connected to the output node or the following hidden layer [23]. Due to all these relationships, it is extremely difficult up to almost impossible to evaluate the importance of individual parameters in the model or to assess how a model 
reached a certain prediction [17]. Therefore, thought the model can fit extremely complex non-linear relationships, this comes at the cost of a low transparency [24].

\subsubsection{Studied clinical examples}

In Chapter 4, we developed a logistic regression model to predict outcome after six months in traumatic brain injury patients admitted to the intensive care unit. For this problem, multiple parameters were used which were proven or suspected to be indicative of the outcome. To obtain high transparency, we used a logistic regression model. This model proved to accurately predict the six-month outcome while we were able to evaluate importance of individual parameters. Therefore, implementation of this model in the clinic is a viable option after external validation. In Chapter 5 and 6 on the other hand, we addressed the prediction of pending syncope in hypovolemic subjects using a support vector machine model and a neural network. Although the models in Chapter 5 and 6 give a fairly accurate prediction of a complex problem, the complexity of the models hampers interpretability and clinical use $[14,30]$. As the model cannot explain why a certain prediction is made (not transparent), it is hard for a clinician to trust the model [31]. The clinician cannot evaluate how the model comes to a certain prediction; this implicates that treatment based on the model prevents the use of knowledge or common sense. An example in which the prediction of a model contradicted common clinical knowledge is found in the prediction of mortality in patients with pneumonia. Data from patients admitted to the hospital with pneumonia was used to train multiple models predicting the probability that the patient would die [32]. A highly accurate neural network model significantly outperformed a rule-based model. Further evaluation of the developed models identified a rule in the rule-based model that patients with pneumonia and asthma were less likely to die [33]. It is known that asthma is not a protective factor, but a risk factor for patients with pneumonia. Hence, patients with asthma and pneumonia were directly admitted to the intensive care unit and therefore received more care, resulting in a lower mortality rate compared to other pneumonia patients. The neural network probably also trained this pattern but due to the black-box nature of the model, the authors were not able to identify this pattern. Relying solely on the prediction of the neural network for mortality in patients with pneumonia would result in severe undertreatment of patients with pneumonia and asthma. This stresses the importance of transparency of clinical models.

\subsection{General considerations}

\subsubsection{What this thesis adds}

We discussed three crucial steps in the process of clinical model creation. First, data quality in medicine is generally low and subject to noise, which may lead to bias and incorrect classifications. Therefore, emphasis has to be given to data preparation and cleaning, with an understanding of the underlying physiology generating the data. Second, we discussed the importance of adequate parameter extraction. Especially in medicine, an important step is the creation of parameters based on physiology. Although the field of machine learning moves towards automated feature engineering, we emphasize the need for interpretable 
parameters if possible. Third, we discussed model creation. We explored the trade-off between accuracy of the model and interpretability of the model. We have shown that a simple logistic regression model may be sufficient for improved prediction, whilst still maintaining interpretability based on clinical parameters. Only if more complex models make a significant better prediction and interpretation is not mandatory, they are warranted to use. Although a model may perform 100\% correctly, it is useless if not implemented due to lack of transparency. Therefore, the initial step should be the creation of transparent and interpretable models.

\subsubsection{Application}

Application of machine learning models in medicine progresses more and more towards complex models such as neural networks [17]. Complex neural networks have been shown to diagnose patients as well or even better than expert clinicians in several cases [34-37]. This opens up the question if machine learning models will eventually replace clinicians. The concern of replacement is voiced especially in the field of radiology, as models process images much faster and possibly more accurate. However, several hurdles prevent machine learning to replace clinicians, such as liability and patient comfort [38]. Furthermore, concern has been voiced that machine learning is not able to take context regarding the underlying physiology into account, as exemplified in the pneumonia combined with asthma case [39]. Therefore, it is probable and wishful that machine learning will be applied to aid the clinician instead of replace the clinician.

One promising area of machine learning models is the self-assessment of patients without visiting a physician. An example given is automatic detection of possible melanoma using a picture made at home by the patient [40]. However, population wide self-assessment requires high sensitivity often resulting in a high false positive rate and false referrals [41]. Hence, before implementation of machine learning algorithms, they have to be further developed to ensure a low false positive rate.

\subsubsection{Limitations}

In this thesis, we discussed several crucial steps to obtain an accurate model for diagnostic or predictive purposes. However, we did not discuss the impact of other aspects during model creation such as feature selection [15], sample size [42,43], ensemble methods [44], ethics [45] or regulation by law [46], to name a few. Each of these aspects is important on its own and requires attention during the process. Furthermore, we only discussed supervised classification models. Unsupervised learning, which aims to find patterns in data without supplying labels, is an example of a different branch of machine learning models that is also important in medicine [47]. In addition, regression instead of classification models have their applications in medicine too. The crucial steps addressed in this thesis also apply to these different areas of machine learning models in medicine. 


\subsection{Conclusion}

Machine learning models will have an enormous impact on the future of medicine. To ensure that such models are developed correctly and will be implemented, it is crucial to obtain data of high quality, to extract parameters based on underlying physiology and to use the correct model. Such models should be trained by a data scientist in collaboration with a clinician, or by someone who has an understanding of the clinical implementation as well as of machine learning models. Furthermore, we stress the importance to keep models transparent, as this is needed for a clinician to trust the model. Without trust, a model will have no application in clinic. 


\subsection{References}

1. Kortekaas MC, Niehof SP, Van Velzen MHN, Galvin EM, Stolker RJ, Huygen FJPM. Comparison of bilateral pulse arrival time before and after induced vasodilation by axillary block. Physiol Meas. 2012;33(12):1993-2002.

2. Babchenko A, Davidson E, Adler D, Ginosar Y, Kurz V, Nitzan M. Increase in pulse transit time to the foot after epidural anaesthesia treatment. Med Biol Eng Comput. 2000;38(6):674-9.

3. Amirtharaj CR, Palmeri LC, Gradwohl G, Adar Y, Nitzan M, Gruber D, et al. Photoplethysmographic assessment of pulse transit time correlates with echocardiographic measurement of stroke volume in preterm infants with patent ductus arteriosus. J Perinatol. 2018;38(9):1220-6.

4. Kim YU, Kim DH, Cheong Y, Kong Y, Lee J, Park SK, et al. Pulse Transit Time as a Predictor of the Efficacy of a Celiac Plexus Block in Patients With Chronic Intractable Abdominal Pain. Clin J Pain. 2016;32(6):522-6.

5. Vlahandonis A, Biggs SN, Nixon GM, Davey MJ, Walter LM, Horne RSC. Pulse transit time as a surrogate measure of changes in systolic arterial pressure in children during sleep. J Sleep Res. 2014;23(4):406-13.

6. Bolea J, Lázaro J, Gil E, Rovira E, Remartínez JM, Laguna P, et al. Pulse Rate and Transit Time Analysis to Predict Hypotension Events After Spinal Anesthesia During Programmed Cesarean Labor. Ann Biomed Eng. 2017;45(9):2253-63.

7. Atla A, Tada R, Sheng V, Singireddy N. Sensitivity of different machine learning algorithms to noise. J Comput Sci Coll. 2011;26(5):96-103.

8. Nettleton DF, Orriols-Puig A, Fornells A. A study of the effect of different types of noise on the precision of supervised learning techniques. Artif Intell Rev. 2010;33(4):275306.

9. Ruzicka M, Akbari A, Bruketa E, Kayibanda JF, Baril C, Hiremath S. How Accurate Are Home Blood Pressure Devices in Use? A Cross-Sectional Study. Guerrot D, editor. PLoS One. 2016;11(6):e0155677.

10. Rinfret F, Cloutier L, Wistaff R, Birnbaum LM, Ng Cheong N, Laskine M, et al. Comparison of Different Automated Office Blood Pressure Measurement Devices: Evidence of Nonequivalence and Clinical Implications. Can J Cardiol. 2017;33(12):1639-44.

11. Gianfrancesco MA, Tamang S, Yazdany J, Schmajuk G. Potential Biases in Machine Learning Algorithms Using Electronic Health Record Data. JAMA Intern Med. 2018;178(11):1544-1547.

12. Brindle PM, McConnachie A, Upton MN, Hart CL, Davey Smith G, Watt GCM. The accuracy of the Framingham risk-score in different socioeconomic groups: a prospective study. Br J Gen Pract. 2005;55(520):838-45.

13. Perel PA, Olldashi F, Muzha I, Filipi N, Lede R, Copertari P, et al. Predicting outcome after traumatic brain injury: practical prognostic models based on large cohort of international patients. BMJ. 2008;336(7641):425-9.

14. Wang F, Casalino LP, Khullar D. Deep Learning in Medicine-Promise, Progress, and Challenges. JAMA Intern Med. 2019;179(3):293-4. 
15. Guyon I, Elisseeff A. An Introduction to Variable and Feature Selection. J Mach Learn Res. 2003;3:1157-82.

16. Foster KR, Koprowski R, Skufca JD. Machine learning, medical diagnosis, and biomedical engineering research - commentary. Biomed Eng Online. 2014;13(94):1-9.

17. Hinton G. Deep Learning-A Technology With the Potential to Transform Health Care. JAMA. 2018;320(11):1101-2.

18. Winkler JK, Fink C, Toberer F, Enk A, Deinlein T, Hofmann-Wellenhof R, et al. Association Between Surgical Skin Markings in Dermoscopic Images and Diagnostic Performance of a Deep Learning Convolutional Neural Network for Melanoma Recognition. JAMA Dermatology. 2019;155(10):1135-41.

19. Deo RC. Machine Learning in Medicine. Circulation. 2015;132(20):1920-30.

20. Mitchell TM. Machine learning. First. New York: McGraw-Hill; 1997.

21. Beam AL, Kohane IS. Big Data and Machine Learning in Health Care. JAMA. 2018;319(13):1317-8.

22. Shipe ME, Deppen SA, Farjah F, Grogan EL. Developing prediction models for clinical use using logistic regression: an overview. J Thorac Dis. 2019;11(S4):S574-84.

23. Hastie T, Tibshirani R, Friedman J. The Elements of Statistical Learning: Data Mining, Inference, and Prediction. Second. New York: Springer-Verlag; 2009.

24. Tu J V. Advantages and disadvantages of using artificial neural networks versus logistic regression for predicting medical outcomes. J Clin Epidemiol. 1996;49(11):1225-31.

25. Cortes C, Vapnik V. Support-Vector Networks. Mach Learn. 1995;20(3):273-97.

26. Navia-Vázquez A, Parrado-Hernández E. Support vector machine interpretation. Neurocomputing. 2006;69(13-15):1754-9.

27. Breiman L, Friedman J, Olshen R, Stone C. Classification and regression trees. First. Boca Raton: Chapman \& Hall/CRC; 1984.

28. Perkins GD, Handley AJ, Koster RW, Castrén M, Smyth MA, Olasveengen T, et al. European Resuscitation Council Guidelines for Resuscitation 2015. Resuscitation. 2015;95:81-99.

29. Bishop CM. Neural networks for pattern recognition. Oxford: Clarendon Press; 1995.

30. He J, Baxter SL, Xu J, Xu J, Zhou X, Zhang K. The practical implementation of artificial intelligence technologies in medicine. Nat Med. 2019;25(1):30-6.

31. Vellido A. The importance of interpretability and visualization in machine learning for applications in medicine and health care. Neural Comput Appl. 2019;1-15.

32. Cooper GF, Abraham V, Aliferis CF, Aronis JM, Buchanan BG, Caruana R, et al. Predicting dire outcomes of patients with community acquired pneumonia. J Biomed Inform. 2005;38(5):347-66.

33. Caruana R, Lou Y, Gehrke J, Koch P, Sturm M, Elhadad N. Intelligible Models for HealthCare: Predicting Pneumonia Risk and Hospital 30-Day Readmission. Proceedings of the 21th ACM SIGKDD international conference on knowledge discovery and data mining. 2015;1721-30.

34. Haenssle HA, Fink C, Schneiderbauer R, Toberer F, Buhl T, Blum A, et al. Man against machine: diagnostic performance of a deep learning convolutional neural network for dermoscopic melanoma recognition in comparison to 58 dermatologists. Ann Oncol. 2018;29(8):1836-42. 
35. Yu K-H, Zhang C, Berry GJ, Altman RB, Ré C, Rubin DL, et al. Predicting non-small cell lung cancer prognosis by fully automated microscopic pathology image features. Nat Commun. 2016;7(1):12474.

36. Ehteshami Bejnordi B, Veta M, Johannes van Diest P, van Ginneken B, Karssemeijer N, Litjens G, et al. Diagnostic Assessment of Deep Learning Algorithms for Detection of Lymph Node Metastases in Women With Breast Cancer. JAMA. 2017;318(22):2199210.

37. Gulshan V, Peng L, Coram M, Stumpe MC, Wu D, Narayanaswamy A, et al. Development and Validation of a Deep Learning Algorithm for Detection of Diabetic Retinopathy in Retinal Fundus Photographs. JAMA. 2016;316(22):2402-10.

38. Recht M, Bryan RN. Artificial Intelligence: Threat or Boon to Radiologists? J Am Coll Radiol. 2017;14(11):1476-80.

39. Cabitza F, Rasoini R, Gensini GF. Unintended Consequences of Machine Learning in Medicine. JAMA. 2017;318(6):517.

40. Rajkomar A, Dean J, Kohane I. Machine Learning in Medicine. N Engl J Med. 2019;380(14):1347-58.

41. Kanagasingam Y, Xiao D, Vignarajan J, Preetham A, Tay-Kearney M-L, Mehrotra A. Evaluation of Artificial Intelligence-Based Grading of Diabetic Retinopathy in Primary Care. JAMA Netw Open. 2018;1(5):e182665.

42. Peduzzi P, Concato J, Kemper E, Holford TR, Feinstem AR. A simulation study of the number of events per variable in logistic regression analysis. J Clin Epidemiol. 1996;49(12):1373-9.

43. Andrew E. Teschendorff. Avoiding common pitfalls in machine learning omic data science. Nat Mater. 2019;18(5):410-27.

44. Dietterich TG. Ensemble Methods in Machine Learning. In: Multiple classifier systems. Berlin: Springer-Verlag; 2000. p. 1-15.

45. Beil M, Proft I, van Heerden D, Sviri S, van Heerden PV. Ethical considerations about artificial intelligence for prognostication in intensive care. Intensive Care Med Exp. 2019;7(1):70.

46. Carter SM, Rogers W, Win KT, Frazer H, Richards B, Houssami N. The ethical, legal and social implications of using artificial intelligence systems in breast cancer care. The Breast. 2020;49:25-32.

47. Castela Forte J, Perner A, van der Horst ICC. The use of clustering algorithms in critical care research to unravel patient heterogeneity. Intensive Care Med. 2019;45(7):1025-8. 



\section{Appendix A}

Summary 
116 | Appendix A 


\section{A.1 Machine learning in medicine}

Machine learning models can outperform clinicians both in accuracy and speed and do not suffer from emotion-based decision making or fatigue. Despite the high potential of machine learning models for clinical application, actual clinical implementation is much lower. This discrepancy may be due to several reasons, amongst others the trust and reliance of clinicians in the particular model. Several factors may affect model accuracy and clinicians' trust.

The aim of this thesis was to analyse the importance of three small but crucial steps inherent to model creation and implementation in medicine. First, we addressed the importance of raw data assessment. Second, we identified the need for adequate and physiology-based parameter extraction. Third, we evaluated the importance of model selection.

\section{A.2 Raw data assessment}

In Chapter 2 we exemplified the importance of raw data assessment in pulse transit time (PTT) measurements, i.e. in the estimations of the delay between the R peak in the ECG and the upslope in the plethysmogram. In patients and in simulations, a constant increase in PTT followed by a sudden decrease after a set time interval was observed. This behaviour of PTT was the result of minimal prolongation of each plethysmogram waveform followed by an abrupt shortened single wave. Since the ECG did not exhibit this pattern, the prolongation and sudden shortening of the plethysmogram waveform resulted in a sawtooth artifact. Although PTT is a commonly used clinical parameter, the sawtooth artifact is large enough to prevent clinical usefulness. Our finding emphasizes the need for continuous data quality monitoring because clinically accepted parameters may still contain severe artifacts.

\section{A.3 Parameter extraction}

Parameter quality is of the utmost importance for model accuracy. High quality parameters can be developed using knowledge of the underlying physiology. We exemplified the need for adequate and physiology-based parameters in Chapter 3 by developing a parameter that can discriminate between preterm infants with or without a patent ductus arteriosus. The ductus arteriosus is a connecting vessel between the aortic arch and the pulmonary artery that should close after birth. If the ductus arteriosus does not close after birth, which often occurs in preterm infants, it is called a patent ductus arteriosus. Due to the positive pressure difference between the aorta and the pulmonary artery, a patent ductus arteriosus shunts blood from the systemic to the pulmonary circulation and, hence, may influence systemic blood pressure. Based on this knowledge, we hypothesized that the ratio of the upward - and downward slope arterial blood pressure at the borders of a given window surrounding the systolic peak can discriminate between patients with and without a patent ductus arteriosus. Our proposed parameter predicted the presence of a patent ductus arteriosus in preterm infants with an accuracy of $83.3 \%$. We conclude that development of high-quality physiologybased parameters is important to improve clinical model accuracy. 


\section{A.4 Model creation}

The outcome in severe traumatic brain injury patients admitted to the intensive care unit ranges from full recovery to death. It is helpful for medical personnel to know in advance how well a patient may recover, as this may indicate how aggressive the patient should be treated. In Chapter 4 we created a logistic regression model to predict whether a severe traumatic brain injury patient admitted to the intensive care unit has a favourable or unfavourable outcome after six months. The prediction was based on physiology-based parameters measured during the first six hours after admission. These parameters were shown or suspected to be indicative of the outcome. The logistic regression model was able to obtain a prediction accuracy of $86.7 \%$. The high accuracy obtained indicates that a simple linear model may be sufficient to accurately predict the outcome in heterogenous groups like severe traumatic brain injury patients. The transparency of the logistic regression model enables the clinician to assess the importance of individual parameters according to their clinical reasoning. Due to the high prediction accuracy, high interpretability and logical reasoning of the model, clinicians may trust and implement this model in their practice. We conclude that models can be accurate and simple at the same time, resulting in a clinically useful model that can be implemented and trusted by clinicians.

Although simple models such as logistic regression models can result in accurate predictions, some clinical problems do require a more sophisticated approach. One of such clinical problems is the detection of pending cerebral hypovolemia due to progressive central hypovolemia, for instance due to bleeding during surgery. In Chapter 5 and 6 we induced central hypovolemia in healthy volunteers up to the point of cerebral hypovolemia as shown by symptoms of syncope. A neural network and a support vector machine were used to predict on a beat-to-beat base how far a subject was from cerebral hypovolemia. It was found that impending syncope could be fairly accurately estimated using these two models that implemented a variety of continuously measured parameters. However, due to the nature of these models, interpretability was low. Therefore, the model and the prediction are interesting, but clinical implementation may lag behind due to the fact that the clinician cannot understand and evaluate the reasoning of the model. We conclude that not only model accuracy, but also model interpretability, transparency and possible implementation should be leading indicators for model selection.

This thesis ends with a general discussion in Chapter 7 in which the various chapters are put into a broader perspective. 




\title{
Appendix B
}

\author{
Valorisation
}


122 | Appendix B 
In general, it is promising that a high number of machine learning models have been developed that potentially might be of relevance in medicine. However, only a few of these models are actually implemented. One of the most important reasons for this discrepancy is the lack of trust by the clinician in models, either because they are non-transparent with unclear algorithms and aims, or because some of these models actually act opposite to clinical reasoning. Because non-implemented models are irrelevant and a waste of energy and resources, machine-learning models for medical applications should be developed according to principles that allow implementation. This thesis addressed several of these principles and is therefore relevant for patient, clinicians and the society in general.

\section{B.1 Relevance for patients}

Patients directly benefit from a high quality of care. If a machine-learning model outperforms a clinician, implementation of this machine-learning model may lead to an increase in quality of medicine. Important gains are more accurate and earlier diagnosis, as well as treatment tailored to the individual. In Chapter 5 and 6, we developed a machine-learning model that may aid the patient by early recognition of central hypovolemia and, consequently, start of treatment in an early stage thereby potentially avoiding detrimental effects such as brain ischemia. Though the developed model can be of direct relevance to the patient, it is not transparent, limiting understanding how the model comes to a prediction with the associated risk of not being implemented in clinical practice. Therefore, it is important to test this model extensively and under various conditions, to ensure that clinicians can rely on the model, trust the model and therefore are at ease before implementing the model. In Chapter 4, we developed a machine-learning model that can predict the outcome of traumatic brain injury patients after six months within six hours after admission. Although not investigated in this study, it can be imagined that treatment may be altered based on the probable outcome, which may directly benefit the patient. Therefore, the next step is to study if such a prediction can be updated during hospitalization, after which clinicians can base treatment intensity on such a prediction to improve patient outcome. Both studies may improve care and benefit patient health, but this mandates further clinical evaluation.

\section{B.2 Relevance for clinicians}

A machine-learning model may assist clinicians in numerous ways. By automating standard tasks such as the evaluation of imaging data, a machine-learning model may severely decrease the workload of clinicians. For instance, the clinician may see only a selection of images that are classified by an algorithm as containing potential disease. Due to the lower workload, clinicians may distribute their time differently, e.g. by spending more time on contact with patients. Since machine-learning models can analyse more patient cases than a clinician will see in his/her whole life, classification can also be based on an even wider range of 'expertise'. Machine-learning models may also give additional insight into underlying physiology. Especially unsupervised machine-learning models have this potential, for instance by finding clusters in patients with similar symptoms but different responses to treatment. Such grouping may lead to new insights for the clinician regarding related physiology. This thesis addressed several crucial steps such as data quality (Chapter 2) and parameter identification (Chapter 
3). By focusing on these steps, model quality and implementation may improve, resulting in the discussed beneficial effects for clinicians. However, numerous other crucial steps are not yet addressed and should be evaluated further, starting with ethics and legislation as they may severely hamper implementation.

\section{B.3 Relevance for society}

The cost of healthcare keeps increasing, resulting in a burden on society. In Chapter 4-6, we developed machine-learning models that may lead to earlier and more effective treatment. Such models are based on physiology-based parameters and artefact-free data, addressed in Chapter 2 and 3. As a result, the cost of healthcare may decrease by for instance 1) less time spend in the hospital by the patient, 2) automatization of tasks and subsequent lowering of the number of clinicians, and 3) screening by patients themselves at home. Home-screening might benefit society because it may result not only in a lower number of unnecessary physician visits but also in less patients with a disease that do not go to a physician. Since these outcomes are hypothetical, research should first be focused on safety and efficacy of machine learning models for home screening and healthcare in general. 




\section{Appendix C}

\section{Dankwoord / Acknowledgements}


128 | Appendix C 


\section{C.1 Dankwoord}

Bij aanvang van mijn $\mathrm{PhD}$ leek vier jaar een eeuwigheid, maar achteraf gezien is het een ontzettend korte tijd. Komend vanuit de studie Technische Geneeskunde, heb ik mij veel technische aspecten proberen eigen te maken en te combineren met de kliniek. Dit is denk ik aardig gelukt, maar niet zonder de hulp, steun en gezelligheid van een grote groep mensen. Waar kan ik deze mensen beter bedanken dan in mogelijk het meest gelezen hoofdstuk van mijn thesis, te weten het dankwoord, startend met mijn promotoren Tammo, Boris en Peter.

Beste Tammo, ik heb veel geleerd van je gedurende deze vier jaar. Bij het werken aan een artikel konden wij inhoudelijk altijd wel door een deur, maar tekstueel werd er altijd veel gediscussieerd. Je fameuze rode pen kreeg dan een eigen geest en waarde op die manier dwars door mijn werk; Het werd er wel altijd weer iets beter door. Ik heb daarom hier de 'fog index' bewust laag proberen te houden. Je hebt mij altijd flink laten doorpakken, tot ik zei "Dit kan ik gewoon niet maken Tammo", en dan probeerde je het toch nog een keertje... Bedankt voor de vele leerzame maar ook gezellige momenten.

Beste Boris, ik heb genoten van het enthousiasme dat je altijd tentoonspreidde, en waarmee je mij ook aanstak. Menigmaal bespraken wij de resultaten van een analyse, waar ik niet al te veel heil in zag maar waar je mij toch weer voor enthousiasmeerde. Daarnaast heb je mij kennis laten maken met veel leuke Duitse gezegdes, die ik met mijn beperkte kennis van de taal helaas ook meteen weer vergeten ben. Hopelijk kun je ze mij nog eens vertellen, dan zal ik ze opschrijven. Vielen Dank für die lehrreichen und lustigen Momente (met dank aan Google Translate)!

Beste Peter, je hebt mij geleerd om de problemen niet enkel technisch te benaderen, maar vooral ook naar een klinische interpretatie te streven. Daarnaast waardeer ik het dat je altijd een rustige benadering had, waardoor ik mezelf niet voorbij liep maar focuste op de dingen goed gedaan krijgen. Jij was ook de eerste van wie ik de "sandwich" methode voor kritiek leveren hoorde (en die dit ook daadwerkelijk toepaste): Ik stond perplex dat dit daadwerkelijk werkte. Bedankt voor de afgelopen jaren en de vele geleerde lessen!

Prof. Peeters, prof. Van der Horst, prof. Mess, prof. Orlikowsky en prof. Veltink, bedankt dat jullie bereid waren om als leescommissie op te treden en voor jullie snelle reactie.

Ben, het blijft mij verbazen hoe goed je met kleuren en figuren bent, helemaal gezien je niet eens kleuren kan zien. Menig keer heb ik een figuur aan je laten zien, waar je mij óf tips gaf om deze te verbeteren, óf aangaf dat deze niet meer te redden viel... Wanneer er tijdens de verdediging vragen komen over de opmaak van de thesis zal ik deze dus door schuiven ;).

Thomas, onze vriendschap is met recht de langste en mogelijk de beste die ik heb. Al sinds groep drie hebben wij het altijd weer gezellig, hoewel de kwaliteit van humor, met name op pokeravonden, toch wel rond het niveau groep drie is blijven hangen! Buiten dit kan ik echter ook met serieuzere zaken bij je aankloppen, waarvoor mijn dank. Laten we dit vooral volhouden! 
Als je vier jaar op een plek wil volhouden, moet de werkplek wel ontzettend leuk zijn. Hiervoor wil ik alle mensen uit het lab bedanken, en iedereen die er tijdelijk is geweest. Aurore, Myrthe, Letty, Frank, bedankt voor de chocolaatjes en gezelligheid op, maar ook buiten het werk. Vaak werd er koffie gehaald voor een praatje of voor de laatste roddels van Temptation Island. Maarten, Sjeng, er zijn een hele hoop redenen om jullie te bedanken (mooie discussies, voetbal sessies), maar voornamelijk dat de gezelligheid niet stopte na werktijd, ook nu nog niet. Daarnaast wil ik ook Job, Erik, Tijmen, Nick, Pamir, Raoul, Lauren, Niek, Peter, Jeroen, Tim, Anneloes, Claire, Lian, Shaiv, Mehrdad, Wouter, Joost, Koen en Koen bedanken voor alle gezelligheid, discussies en praatjes die we de afgelopen jaren hebben gehad.

Frank, Timon, als onderdeel van TBFF hebben we de master fase van Technische Geneeskunde doorlopen, waarbij we altijd goed scoorden samen. Wat echter echt bij blijft is de gezelligheid eromheen, want het was simpelweg altijd leuk. Er staat nog een uitje open: Hopelijk kunnen we binnenkort daarmee verder!

Beste Carola, ik durf te wedden dat je af en toe gek van me werd als ik weer vroeg om meer data, of je specifiek dit wilde extraheren van de database, of je dit kon uitzoeken, waarom deze patiënt zo was opgeslagen etc. Toch deed je dit wel altijd voor me, en ik heb onze samenwerking als heel fijn ervaren. Bedankt voor de vele hulp over de jaren.

Beste Marcel, bedankt voor de begeleiding en de hulp bij ons gezamenlijke onderzoek. Het was altijd interessant om met je te sparren; Meestal ontstonden er tijdens een gesprek spontaan nieuwe ideeën. Wie weet zit een samenwerking in de toekomst er nog een keer in. Voorlopig succes met Hersenstrijd!

Pietro, bedankt dat ik altijd met vragen over specifieke technieken naar jou toe kon en dat je mij hierbij uitgebreid hielp.

Han, Björn, onder jullie leiding ben ik begonnen aan mijn specialisatie richting machine learning in medicine, wat getuige dit boekje een goede keuze blijkt te zijn geweest. Bedankt voor de hulp en de steun hierbij.

Alle co-auteurs die ik hierboven nog niet heb genoemd, wil ik bedanken voor de input en de hulp bij de artikelen.

De afgelopen jaren heb ik meerdere stagiaires mogen begeleiden, die mij goed lieten nadenken over de stof, waarvoor mijn dank. Speciale dank gaat uit naar Bibi, die de basis heeft gelegd waar ik hoofdstuk 4 verder op kon bouwen.

Vriendjes en vriendinnetjes, bedankt voor de gezellige tijden die jullie me altijd gaven als ik mij weer richting het Noorden begaf. Ik ken vele van jullie al sinds de middelbare school, en nog altijd breidt de groep zich verder uit (welkom Julian) en wordt het gezelliger en gezelliger. Wacht maar af, wij komen er weer aan! 
Borrelbuddies, als er ergens goede bonding voor de kinderen en aanhang is, is het wel bij ons. De borrels zijn ondertussen een begrip, en het is goed te weten dat ik ook bij jullie terecht kan voor gezelligheid en steun. Want je weet: Borrelbuddies, borrelbuddies, steunen elkaar!

De afgelopen vier jaar zouden nooit volbracht kunnen zijn als ik de 25 voorgaande jaren niet een perfecte basis had gekregen. Pap, mam, bedankt dat jullie mijn jeugd en leven altijd tot een plezier hebben gemaakt. Jullie hebben mij gestimuleerd op de juiste momenten, maar nooit gepusht. Er zijn zoveel dingen die jullie hebben gedaan die ik waarschijnlijk niet eens door heb gehad, of anders nooit mijn dank voor heb uitgesproken. Bedankt voor alles.

Pippie en Kareltje, jullie hebben toch wel een speciaal plekje in mijn hart veroverd. Jullie maakten de tijd in Maastricht nog gezelliger dan het al was. Het is zelfs zo erg dat ik een foto van jullie op het lab had hangen, terwijl mijn eigen vriendin er niet hing... Mijn favoriete katten!

Lieve Laura, toen ik zei dat ik naar Maastricht ging, ben je meteen meegegaan. Ik heb nog nooit zo duidelijk het gevoel gehad dat ik altijd, zonder twijfel, bij iemand terecht kan en op iemand terug kan vallen. Iedere keer weet je mij weer te verrassen, soms met een simpele borrel, soms met een uitgebreide scootertocht, soms met een reis naar Cambodja (wie had gedacht dat ik daar ooit heen zou gaan)! Laura, ik ben nog altijd gek op je; laten we samen verder gaan op een nieuw avontuur. 



\section{Appendix D}

Curriculum Vitae 
134 | Appendix D 


\section{About the author}

Frank Bennis was born on the 23rd of July 1991 in Zwaag, the Netherlands. In 2009 he obtained his VWO degree at Oscar Romero in Hoorn.

In September 2009, Frank started with the study Technical Medicine at the University of Twente. He obtained his Bachelor's degree in 2012, after which he started with the Master track Medical Sensing and Stimulation. As part of the Master track he performed clinical internships at the Clinical Neurophysiology department at the Medisch Spectrum Twente, the Cardiology department at Radboud UMC, the Epilepsy unit at the Stichting Epilepsie Instellingen Nederland and the lab for Clinical Cardiovascular Physiology at Academisch Medisch Centrum Amsterdam. Frank continued the latter internship for his graduation internship, during which he developed a neural network predictive of pending syncope (see chapter five).

After graduation, Frank started with his $\mathrm{PhD}$ at the department of Biomedical Engineering at the University of Maastricht under supervision of prof. dr. Delhaas, prof. dr. Kramer and dr. Andriessen (Maxima Medisch Centrum Veldhoven). Frank focused on the development of machine learning techniques for healthcare, amongst others in the (neonatal) intensive care unit, which led to the current $\mathrm{PhD}$ thesis.

As of September 2020, Frank is working as a postdoctoral researcher at the Vrije Universiteit Amsterdam under direct supervision of dr. Hoogendoorn. 


\section{Publications}

Bennis FC, van der Ster BJ, van Lieshout JJ, Andriessen P, Delhaas T. A machine-learning based analysis for the recognition of progressive central hypovolemia. Physiol Meas. 2017;38(9):1791-1801. doi:10.1088/1361-6579/aa7d3d

Bennis FC, Geertsema EE, Velis DN, Reus EE, Visser GH. The use of single bipolar scalp derivation for the detection of ictal events during long-term EEG monitoring. Epileptic Disord. 2017;19(3):307-314. doi:10.1684/epd.2017.0926

Hermans BJM, Vink AS, Bennis FC, et al. The development and validation of an easy to use automatic QT-interval algorithm. PLoS One. 2017;12(9):e0184352. doi:10.1371/journal. pone.0184352

van der Ster BJP, Bennis FC, Delhaas T, Westerhof BE, Stok WJ, van Lieshout JJ. Support Vector Machine Based Monitoring of Cardio-Cerebrovascular Reserve during Simulated Hemorrhage. Front Physiol. 2018;8:1057. doi:10.3389/fphys.2017.01057

Bennis FC, Aries MJH, Delhaas T. Letter by Bennis et al Regarding Article, "Cerebral Near-Infrared Spectroscopy: A Potential Approach for Thrombectomy Monitoring”. Stroke. 2018;49(3):e135. doi:10.1161/strokeaha.117.020293

Hermans BJM, Stoks J, Bennis FC, et al. Support vector machine-based assessment of the T-wave morphology improves long QT syndrome diagnosis. Europace. 2018;20(suppl_3):113-119. doi:10.1093/europace/euy243

Bennis FC, van Pul C, van den Bogaart JJL, Andriessen P, Kramer BW, Delhaas T. Artifacts in pulse transit time measurements using standard patient monitoring equipment. PLoS One. 2019;14(6):e0218784. doi:10.1371/journal.pone.0218784

Bennis FC, Teeuwen B, Zeiler FA, et al. Improving Prediction of Favourable Outcome After 6 Months in Patients with Severe Traumatic Brain Injury Using Physiological Cerebral Parameters in a Multivariable Logistic Regression Model. Neurocrit Care. 2020;10.1007/ s12028-020-00930-6. doi:10.1007/s12028-020-00930-6

Hermans BJM, Bennis FC, Vink AS, et al. Improving long QT syndrome diagnosis by a polynomial-based T-wave morphology characterization. Heart Rhythm. 2020;17(5 Pt A):752758. doi:10.1016/j.hrthm.2019.12.020 